\title{
FEHMN 1.0: Finite Element Heat and Mass Transfer Code
}

\section{Los ANDOMOS}

Los Alamos National Laboratory is operated by the University of Califormia for the United States Dcparfment of Energy under contract W-7405-ENG-36. 
Prepared by Kay Coen, Group EES-5

This work was supportcd by the US Department of Energy, Yucca Mountain Site Characterization Project Office as part of the Civilian Radioactive Waste Management Program.

An Affirmative Action/Equal Opportunity Employer

This report was prepared as an account of wark sponsored by an agency of the United States Gozernment. Neither The Regents of the Universily of California, the United State's Government nor any agency thereof, nor any of their employces, makes any zoarranty, express or implied, or assumes any legal liability or responsibility for the accuracy. completeness, or usefulness of any information, apparatus, product, or process disclosed, or replesents that its use zould not infringe prizately ound rights. Reference herein to any specific commercini product, process, or seroice by trade name, trademark, mamefacturer, or otherwise, dos not necessarily constitute or imply its endorsement, recommendation, or faworing by The Regents of the Umiversity of Califormia, the United States Government, or any agency there of. The riezes and opinions of aulhors expressed herein do not necessarily state or reflect linose of Tire Regents of the Uniersity of Califormia. the United State's Goierment, or any agency thereof. 
FEHMN 1.0: Finite Element Heat

and Mass Transfer Code

George Zyvoloski

Zora Dash

Sharad Kelkar 


\section{Contents}

FIGURES ......................... . . . . . . . .

TABLES ........................ . . . . . . . . . . . .

ABSTRACT . . . . . . . . . . . . . . . . . . . 1

I. MODEL DESCRIPTION . . . . . . . . . . . . . . . . . . . . . . . . . 1

A. Nature and Purpose . . . . . . . . . . . . . . . . . . . . . . . 1

B. Mathematical Formulation . . . . . . . . . . . . . . . . 2

C. Discretization of the Governing Equations . . . . . . . . . . . . . . . 14

D. Boundary Conditions . . . . . . . . . . . . . . . . 17

E. Solution Methods . . . . . . . . . . . . . . . . . . . . 17

F. Dual Porosity Formulation . . . . . . . . . . . . . . . . 21

II. CODE DESCRIPTION . . . . . . . . . . . . . . . . . . . . . 25

A. Subroutines . . . . . . . . . . . . . . . . . 25

B. Common Block and Parameter Statements . . . . . . . . . . . . . . 33

C. Subroutine Structure for FEHMN . . . . . . . . . . . . . . . 47

III. USER'S MANUAL . . . . . . . . . . . . . . . . . . . . . . 51

A. Automatic Mesh Generation . . . . . . . . . . . . . . . . . . . 51

B. Macro Command Input for FEHMN . . . . . . . . . . . . . . . . . . . 56

C. Graphics Postprocessing . . . . . . . . . . . . . . . . . . . . . 76

D. Example Input Files and Output Files . . . . . . . . . . . . . . . 80

ACKNOWLEDGMENTS . . . . . . . . . . . . . . . . . . . . . . 111

DISCLAIMER . . . . . . . . . . . . . . . . . . . 111

REFERENCES ....................... . . . 111 


\section{FIGURES}

1. Comparison of nodal connections for conventional and Lobatto integrations. . . . 16

2. Matrix band structures showing second-order fill-in.

$x$ - original matrix positions.

o - fill-in with order 2 factorization. . . . . . . . . . . . . . . . . . 20

3. Computational volume element showing dual porosity parameters . . . . . . . 22

4. Structure for subroutine calls for FEHMN. . . . . . . . . . . . . . . . . . 48

5. Elements available with FEHMN in 2-D and 3-D problems showing numbering convention. . . . . . . . . . . . . . . . . . . . 52

6. Nodal ordering for defining 2-D and 3-D domain blocks for GENMSH Input. . . . 53

7. Terminal output for a FEHMN run on a CRAY computer. . . . . . . . . . . 59

8. Schematic diagram of the 3-D heat conduction problem. . . . . . . . . . . . 84

9. Input for preprocessor GENMESH for 3-D heat conduction example. . . . . . . 84

10. Grid for $4 \times 4 \times 4$ heat conduction problem. . . . . . . . . . . . . . . . . 85

11. Input file for FEHMN for 3-D heat conduction example. . . . . . . . . . . . 86

12. Computer output for the 3-D heat conduction example. . . . . . . . . . . . 90

13. Instruction file (HIS.INS) for postprocessor FEHPLTR for the heat conduction problem. . . . . . . . . . . . . . . . 92

14. History plot for the heat conduction problem. . . . . . . . . . . . . . . . 93

15. Solution domain and results for the Toronyi example. . . . . . . . . . . . . 94

16. Input for GENMSH for the Toronyi example. . . . . . . . . . . . . . . . . 94

17. Input file for FEHMN for Toronyi example. . . . . . . . . . . . . . . . . . 95

18. Output for the Toronyi example. . . . . . . . . . . . . . . . . . . . . . 98

19. Schematic diagram of the geometry and boundary conditions for the DOE code comparison project problem. . . . . . . . . . . . . . . . 100

20. Comparison of FEHMN production well temperatures with results from other codes. . . . . . . . . . . . . . . . . . . . . 100

21. Comparison of FEHMN production well pressure with results from other codes. 101

22. Comparison of FEHMN observation well pressure with results from other codes. 101

23. Input for GENMSH for the DOE example. . . . . . . . . . . . . . . . . 102

24. Input for FEHMN for the DOE example. . . . . . . . . . . . . . . . . 102

25. Output from FEHMN for the DOE example. . . . . . . . . . . . . . . 108

26. Instruction file (CON.INS) for the postprocessor FECPLTR for the DOE problem. . . . . . . . . . . . . . . . . . . . . . 109

27. Contour plot of temperature for the DOE problem. . . . . . . . . . . 110 


\section{TABLES}

I. Notation . . . . . . . . . . . . . . . . . . . . . 3

II. Sorption Isotherm Models . . . . . . . . . . . . . . . . . . . . . . . 10

III. Subroutine Calls Within FEHMN . . . . . . . . . . . . . . . . . . . . . 25

IV. Capabilities of FEHMN with Macro Command References . . . . . . . . . . 57

V. FEHMN Files . . . . . . . . . . . . . . . . . . . . 57

VI. Macro Control Statements for FEHMN . . . . . . . . . . . . . . . 60

VII. Input Description for FEHMN . . . . . . . . . . . . . . . . . . . . . 62

VIII. Input Parameters for the 3-D Heat Conduction Problem . . . . . . . . . . . 80

IX. Sample Problems for 3-D Heat Conduction . . . . . . . . . . . . . . . . . 81

X. Comparison of Analytical and Model Resulis for 3-D Heat Conduction . . . . . 81

XI. Input Parameters for Toronyi Example . . . . . . . . . . . . . . . . . . . 82

XII. Input Parameters for the DOE Code Comparison Project . . . . . . . . . . . 83 


\title{
FEHMN 1.0: \\ FINITE ELEMENT HEAT AND MASS TRANSFER CODE
}

by

George Zyvoloski, Zora Dash, and Sharad Kelkar

\begin{abstract}
A computer code is described which can simulate non-isothermal multiphase multicomponent flow in porous media. It is applicable to natural-state studies of geothermal systems and ground-water flow. The equations of heat and mass transfer for multiphase flow in porous and permeable media are solved using the finite element method. The permeability and porosity of the medium are allowed to depend on pressure and temperature. The code also has provisions for movable air and water phases and noncoupled tracers; that is, tracer solutions that do not affect the heat and mass transfer solutions. The tracers can be passive or reactive. The code can simulate two-dimensional, two-dimensional radial, or three-dimensional geometries. A summary of the equations in the model and the numerical solution procedure are provided in this report. A user's guide and sample problems are also included.
\end{abstract}

\section{MODEL DESCRIPTION}

\section{A. Nature and Purpose}

The FEHMN (Finite Element Heat and Mass Nuclear) code, described in this report, is a version of FEHM (Finite Element Heat and Mass, Zyvoloski et al., 1988) developed for the Yucca Mountain Site Characterization Project (YMP) and documented as required by NUREG-0856. This report satisfies the document requirements for model description and user's manual as presented in the LANL YMP Computer Software Control Quality Procedure (SQAP-3.7, R0). The verification and validation reports are produced as separate documents. The main use of FEHMN will be to assist in the understanding of flow fields in the saturated zone below the potential Yucca Mountain repository. This is referred to as the C-Wells project (YMP-LANL-SP-8.3.1.2.3.1.7). Also in regards to that project, the code will be used to design tracer tests (reactive and non-reactive) to characterize the flow field below Yucca Mountain. In addition, FEHMN will be used to study coupled processes (multicomponent and natural convection) in the unsaturated zone (YMP-LANLSCP-8.3.1.3.7.1). We note here that the model requirements may be found in the above mentioned study plans. 
Yucca Mountain is extremely complex both hydrologically and geologically. The computer codes that are used to model flow must be able to describe that complexity. For example, the flow at Yucca Mountain, in both the saturated and unsaturated zones is dominated by fracture and fault flow in many areas. With permeation to and from faults and fractures, the flow is inherently three-dimensional (3-D). Birdsell et al. (1990) recently presented calculations showing the importance of 3-D flow at Yucca Mountain. Coupled heat and mass transport occurs in both the unsaturated and saturated zones. In the far field unsaturated zone, Weeks (1987) has described natural convection that occurs through Yucca Mountain due to seasonal temperature changes. Heat and mass transfer are important in the saturated zone modeling of temperature logs and pressure tests.

The governing equations and solution techniques used in the FEHMN code are described below. In addition, a user's manual with example problems is provided. Future changes to the code and documentation will be made in accordance with quality assurance procedures being established for the Yucca Mountain Site Characterization Project.

\section{B. Mathematical Formulation}

1. Governing equations. Detailed derivations of the governing equations for twophase flow including heat transfer have been presented by several investigators (Mercer and Faust, 1975, and Brownell et al., 1975, for example), and therefore only a brief development will be presented here. The notation used in this report is given in Table I.

Conservation of mass is expressed by the equation

$$
\frac{\partial A_{m}}{\partial t}+\bar{\nabla} \cdot \bar{f}_{m}+q_{m}=0
$$

where the mass per unit volume, $A_{m}$, is given by

$$
A_{m}=\phi\left(S_{v} \rho_{v}+S_{\ell} \rho_{\ell}\right)
$$

and the mass flux, $\bar{f}_{m}$, is given by

$$
\bar{f}_{m}=\rho_{v} \bar{V}_{v}+\rho_{\ell} \bar{V}_{\ell}
$$

Here $\phi$ is the porosity of the matrix, $S_{v}$ and $S_{\ell}$ are saturations, $\rho_{v}$ and $\rho_{\ell}$ are densities, and $\bar{V}_{v}$ and $\bar{V}_{\ell}$ are velocities with the subscripts $v$ and $\ell$ indicating quantities for the vapor phase and the liquid phase, respectively. Source and sink terms (such as bores, reinjection wells, or groundwater recharge) are represented by the term $q_{m}$.

Conservation of fluid-rock energy is expressed by the equation

$$
\frac{\partial A_{e}}{\partial t}+\bar{\nabla} \cdot \bar{f}_{e}+q_{e}=0
$$


A

$[A]$

$A_{c}$

$A_{e}$

$A_{m}$

[ $\tilde{A}]$

$a$

$a_{1}, a_{2}, a_{3}, a_{4}$

$\{b\}$

$C_{\ell}$

$C_{R}$

$C_{v}$

$C_{\max }$

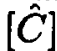

$C_{i j}^{u p}$

$c_{g}$

$c_{p a}$

$c_{r}$

$c p_{1}, c p_{2}$

$c p_{3}, c p_{4}$

$D_{c}$

$\left[D_{c}\right]$

$D_{e \ell}$

$D_{e, v}$

$D_{m \ell}$

$D_{m, v}$

$D_{e}^{u p}$

$D_{m}^{u p}$

$\{e\}$

E

$F$

$\|F\|$

$\left[F_{c}\right]$

$F_{e}$

$F_{m}$

$\bar{F}(\bar{x})$

$\left\{F_{e}\right\}$

$\left\{F_{m}\right\}$

$f(y)$

$g$

$[H]^{m}$ parameter used in nonlinear adsorption model (Freundlich, modified Freundlich) solution matrix for system of nonlinear equations

accumulation term for tracer $\left(M / L^{3}\right)$

energy accumulation term $\left(M /{ }^{2} L\right)$

mass accumulation term $\left(M / L^{3}\right)$

approximation of matrix $[A]$

weighting factor for time discretization

coefficients used in reaction rate model

right hand side (forcing function) for system of linear equations

tracer concentration in liquid

tracer concentration adsorbed on rock

tracer concentration in vapor

parameter used in nonlinear adsorption model (modified Freundlich)

capacitance matrix

upwind value of tracer concentration

parametcr used in linear porosity model

heat capacity of air $L^{2} / \theta^{2} T$

parameter used in linear porosity model

parameters used in the capillary pressure models

parameters used in the capillary pressure models

dispersion coefficient for tracer $\left(L^{2} / \theta\right)$

finite element coefficients for tracer dispersion term

energy transmissibility term for liquid $\left(L^{2} / \theta\right)$

energy transmissibility term for vapor $\left(L^{2} / \theta\right)$

mass transmissibility term for liquid $(\theta)$

mass transmissibility term for vapor $(\theta)$

upwind energy transmissibility term $\left(L^{2} / \theta\right)$

upwind mass transmissibility term $(\theta)$

unit vector

Youngs modulus $\left(M L / \theta^{2}\right)$

function representation

$L^{2}$ norm of residuals

residual for tracer equation

flux vector for energy equation $\left(M / \theta^{3}\right)$

flux vector for mass equation $\left(M / L^{2}\right)$

vector of equation residuals

residual for energy equations

residual for mass equation

intermediate calculation in GMRES acceleration

acceleration of gravity $\left(L / \theta^{2}\right)$

intermediate calculation in GMRES acceleration 
$h \quad$ generalized energy variable $\left(T\right.$ or $\left.L^{2} / \theta^{2}\right)$

$h_{a} \quad$ enthalpy of air $\left(L^{2} / \theta^{2}\right)$

$h_{m}^{m e l} \quad$ parameter in GMRES acceleration

$h_{v}$ mass fraction of air in the vapor phase

$h_{v, w}$ vapor enthalpy $\left(L^{2} / \theta^{2}\right)$

$h_{\ell}$ mass fraction of air in the liquid phase

$h_{\ell, w}$ liquid enthalpy $\left(L^{2} / \theta^{2}\right)$

$I_{i}$ impedance at node $i\left(\theta / L^{2}\right)$ heat flow impedance at node $I\left(M / L^{3} \theta T\right)$

$[K]$ finite element coefficient for heat conduction term

$K_{D}$ retardation coefficient (linear adsorption)

$K_{h, v, w}$ thermal conductivity $\left(M \bar{L} / T \theta^{3}\right)$

$k$ intrinsic rock permeability $\left(L^{2}\right)$

$L_{f}, L_{0} \quad$ length scales used in dual porosity problem

$L_{f 1}, L_{f 2} \quad$ length scales used in dual porosity problem

$[\widetilde{L}]$

$m$ approximate lower factorization of matrix

$[N]$ exponent used in Gangi stress model finite element shape function source term for mass equation $\left(M / \theta L^{3}\right)$ source term for tracer $\left(M / \theta L^{3}\right)$

$q_{e}$ source term for energy equation $\left(M / L^{3} \theta\right)$

$q_{i}$ mass source term at node $i\left(M / \theta L^{3}\right)$ energy source term at node $i\left(M / L^{3} \theta\right)$ coefficient for energy source terms coefficient for mass source terms

$P_{o}$

$P_{\ell}$ closure stress for use in Gangi stress model $(M L)$

$P_{v}$ $P_{\text {cap }}$ initial press'ıre $\left(M L / \theta^{2}\right)$ liquid pressure $\left(M / L \theta^{2}\right)$ $\left[R_{c}\right]$ vapor pressure $\left(M / L \theta^{2}\right)$ capillary pressure $\left(M / L \theta^{2}\right)$ $R_{\ell} \quad$ liquid relative permeability $\left\{R_{e}\right\} \quad$ gravity term coefficient for energy $\left\{R_{m}\right\} \quad$ gravity term coefficient for mass 
$R_{v} \quad$ vapor relative permeability

$\{r\}$ residual of linear system of equations

$r, r_{b}$ parameters used in nonlinear adsorption model (Langmuir)

$r_{p 1}, r_{p 2}$ parameters used in relative permeability models

$r_{p 3}, r_{p 4}$ parameters used in relative permeability models $S$ normalized liquid saturation

$S_{\ell}$ liquid saturation

$\hat{S}_{\ell}$ normalized liquid saturation

$S_{\ell r}$ residual liquid saturation $S_{\ell s}$ maximum liquid saturation $S_{v}$ vapor saturation $T$ $T^{*}$ temperature $(T)$

$\left[T_{e}(P, h)\right] \quad$ stiffness matrix for energy equation $\left[T_{c}(C)\right] \quad$ stiffness matrix for concentration equation $\left[T_{m}(P, h)\right] \quad$ stiffness matrix for mass equation $T_{\text {flow }} \quad$ flowing temperature at node $i(T)$ $T_{f f}, T_{f f 1} \quad$ transfer terms in dual porosity solution $T_{f f 2} \quad$ transfer terms in dual porosity solution $t \quad$ time $(\theta)$

$u_{\ell} \quad$ liquid internal energy $\left(L^{2} / \theta^{2}\right)$

$u_{v}$ $u_{r}$ $V_{f}$ vapor internal energy $\left(L^{2} / \theta^{2}\right)$ rock internal energy $\left(L^{2} / \theta^{2}\right)$ volume fraction for fractures in a dual porosity problem $V_{f 0}, V_{f 1} \quad$ volume fractions used in a dual porosity problem $V_{f 2}$ volume fractions used in a dual porosity problem liquid velocity $(L / \theta)$ vapor velocity $(L / \theta)$ intermediate calculation in GMRES acceleration approximate upper factorization of matrix intermediate calculation of GMRES acceleration parameter in GMRES acceleration intermediate calculations in GMRES acceleration vector of corrections at iteration $i$ coefficient of thermal expansion $(1 / T)$ $\alpha_{1}, \alpha_{2}$ coefficients used in sorption models 
$\varepsilon \quad$ tolerance taken for solution scheme

$\lambda$ parameter used in defining the van Genutchen capillary pressure model

$\mu_{a} \quad$ air viscosity $(M / L \theta)$

$\mu_{\ell} \quad$ liquid phase viscosity $(M / L \theta)$

$\mu_{\ell, w} \quad$ liquid water viscosity $(M / L \theta)$

$\mu_{v} \quad$ vapor phase viscosity $(M / L \theta)$

$\mu_{v, w} \quad$ steam viscosity $(M / L \theta)$

$\phi \quad$ porosity

$\phi_{0} \quad$ initial porosity

$\sigma \quad$ fractional vapor flow parameter

$\sigma \quad$ in situ stress $\left(M L / \theta^{2}\right)$

$\left(\frac{\partial \bar{F}}{\partial \bar{x}^{k}}\right)$ Jacobian matrix for norlinear system

* Units given in ML $\theta \mathrm{T}$ system of dimensions:

Mass $[M]$, length $[L]$, time $[\theta]$, temperature $[T]$

where the energy per unit volume, $A_{\epsilon}$, is given by

$$
A_{e}=(1-\phi) \rho_{r} u_{r}+\phi\left(S_{v} \rho_{v} u_{v}+S_{\ell} \rho_{\ell} u_{\ell}\right)
$$

and the energy flux, $\bar{f}_{e}$, is given by

$$
\bar{f}_{e}=\rho_{v} h_{v} \bar{V}_{v}+\rho_{\ell} h_{\ell} \bar{V}_{\ell}-K \bar{\nabla} T .
$$

Here the subscript $r$ refers to the rock matrix; $u_{r}, u_{v}$, and $u_{\ell}$ are specific internal energies; $h_{v}$ and $h_{\ell}$ are specific enthalpies; $K$ is an effective thermal conductivity; $T$ is the temperature; and $q_{e}$ is the energy contributed from sources and sinks.

To complete the governing equations it is assumed that Darcy's Law applies to the movement of each phase:

$$
\bar{V}_{v}=-\frac{k R_{v}}{\mu_{v}}\left(\bar{\nabla} p_{v}-\rho_{v} \bar{g}\right)
$$

and

$$
\bar{V}_{\ell}=-\frac{k R_{\ell}}{\mu_{\ell}}\left(\bar{\nabla} p_{\ell}-\rho, \bar{g}\right)
$$


Here $k$ is the permeability, $R_{v}$ and $R_{\ell}$ are the relative permeabilities, $\mu_{v}$ and $\mu_{\ell}$ are viscosities, $p_{\ell}$ and $p_{v}$ the phase pressures, and $g$ represents the acceleration due to gravity (the phase pressures are related by $p_{v}=p_{\ell}+p_{c a_{p}}$ ). For simplicity, the equations are shown for an isotropic medium, though this restriction does not exist in the computer code.

Using Darcy's Law the basic conservation equations (1) and (4) can be rewritten

$$
-\bar{\nabla} \cdot\left(D_{m \ell} \bar{\nabla} p_{\ell}\right)-\bar{\nabla} \cdot\left(D_{m \ell} \bar{\nabla} p_{v}\right)+q_{m}+\frac{\partial}{\partial z} g\left(D_{m \ell} \rho_{\ell}+D_{m v} \rho_{v}\right)+\frac{\partial A_{m}}{\partial t}=0
$$

and

$$
\begin{aligned}
-\bar{\nabla} \cdot\left(D_{e \ell} \bar{\nabla} p_{\ell}\right) & -\bar{\nabla} \cdot\left(D_{e v} \bar{\nabla} p_{v}\right)+\bar{\nabla} \cdot(K \bar{\nabla} T)+q_{e}+\frac{\partial}{\partial z} g\left(D_{e \ell} \rho_{\ell}+D_{e v} \rho_{v}\right) \\
& +\frac{\partial A_{e}}{\partial t}=0
\end{aligned}
$$

where $z$ is oriented in the direction of gravity. Here the transmissibilities are given by

$$
D_{e v}=h_{v} D_{m v}, \quad D_{e \ell}=h_{\ell} D_{m \ell}
$$

and

$$
D_{m \ell}=\frac{k R_{\ell} \rho_{\ell}}{\mu_{\ell}}, \quad D_{m v}=\frac{k R_{v} \rho_{v}}{\mu_{v}}
$$

The source and sink terms in equations (1) and (4) arise from bores, and if the total mass withdrawal, $q_{m}$, for each bore is specified, then the energy withdrawal, $q_{e}$, is determined as follows:

$$
q_{e}=q_{v} h_{v}+q_{\ell} h_{\ell}
$$

where

$$
q_{v}=\sigma q_{m}, \quad q_{\ell}=(1-\sigma) q_{m}
$$

and

$$
\sigma=\frac{1}{\left(1+\frac{\rho_{\ell} R_{\ell} \mu_{v}}{\rho_{v} R_{v} \mu_{\ell}}\right)}
$$

The form of equation (15) shows how important the relative permeability ratio $R_{\ell} / R_{v}$ is in controlling the discharge composition. The relative permeability and capillary pressure functions are summarized in the next section, Constitutive Relationships. 
In addition to the flow of heat and mass, FEHMN is also capable of simulating noncondensible gas flow (usually air) and passive tracer flow. The noncondensible gas conservation equation is

$$
\begin{aligned}
& -\bar{\nabla} \cdot\left(C_{\ell} D_{m \ell} \bar{\nabla} p_{\ell}\right)-\bar{\nabla} \cdot\left(C_{v} D_{m v} \bar{\nabla} p_{v}\right) \\
& +\frac{\partial}{\partial z} g\left(C_{\ell} D_{m \ell} \rho_{\ell}+C_{v} D_{m v} \rho_{v}\right)+\frac{\partial A_{c}}{\partial t}+q_{c}=0 .
\end{aligned}
$$

Here $C$ is the concentration of the noncondensible gas and is expressed as a fraction of total mass. The term $\bar{f}_{z}$ is the mass flux, $q_{c}$ is the source (or sink) strength, and $A_{c}$ is the accumulation term:

$$
\begin{aligned}
\bar{f}_{c} & =C_{v} \rho_{v} \bar{V}_{v}+\rho_{\ell} \bar{V}_{\ell} C_{l} \\
q_{c} & =C_{v} q_{v}+C_{\ell} q_{\ell} \\
A_{c} & =\phi\left(S_{v} \rho_{v} C_{v}+S_{\ell} \rho_{\ell} C_{\ell}\right)
\end{aligned}
$$

The passive tracer equation is not directly coupled to the pressure field but merely ises the pressure field obtained by the heat and mass transfer solution:

$$
\begin{gathered}
-\bar{\nabla} \cdot\left(C_{\ell} D_{m \ell} \bar{\nabla} p_{\ell}\right)-\bar{\nabla} \cdot\left(C_{v} D_{m v} \bar{\nabla} p_{v}\right)+\bar{\nabla} \cdot\left(D_{c} \bar{\nabla} C_{\ell}\right) \\
+g \frac{\partial}{\partial z}\left(C_{\ell} D_{m \ell} \rho_{\ell}+C_{v} D_{m v} C_{v}\right)+\frac{\partial A_{c}}{\partial t}+q_{c}=0
\end{gathered}
$$

(where the terms are defined analogously to the condensible gas equation terms) the additional term $\bar{\nabla} \cdot\left(D_{c} \bar{\nabla} C_{\ell}\right)$ is the dispersion term. At present the code allows for up to 10 tracers.

2. Constitutive Relationships. In the equations described above the porosity, permeability, density, enthalpy, and viscosity can be strong functions of pressure and temperature. These functions make the code very nonlinear. In addition the relative permeabilities and capillary pressure can be strong functions of saturation.

The pressure and temperature dependent behavior of the density, enthalpy, and viscosity are represented by rational polynomials. Using a technique developed at the University of Auckland, New Zealand, accurate fits to the National Bureau of Standards (NBS) steam table data were obtained over the ranges:

$$
\begin{aligned}
0.001 M P a & \leq P \leq 110 . M P a \\
0.001^{\circ} \mathrm{C} & \leq T \leq 360^{\circ} \mathrm{C}
\end{aligned}
$$


The maximum error was $0.3 \%$ for all the functions. The rational polynomial representation proved to be both accurate and fast. For details the reader is referred to Zyvoloski and Dash (1991). In addition a low pressure thermodynamics set is provided with the ranges $0.001 \mathrm{MPa} \leq P \leq 20 \mathrm{MPa}, 0.5^{\circ} \mathrm{C} \leq T \leq 360^{\circ} \mathrm{C}$. The maximum error for this set is also $0.3 \%$.

The tracer module of FEHMN has provisions for reactive tracers. At present four adsorption models have been included. These are the linear, Langmuir (Satter et al., 1980), Freundlich, and modified Freundlich isotherm models. The discussion follows the description given by Robinson (1988). With adsorption the equations describing the conservation of species, Eq. (16) is modified by the addition of the term

$$
\rho_{r} \frac{\partial C_{R}}{\partial t}
$$

where $\rho_{r}$ is the rock density and $C_{R}$ represents the adsorption of species onto the reservoir rock and is given by

$$
C_{R}=\frac{\alpha_{1} C_{\ell}^{\beta}}{1+\alpha_{2} C_{\ell}^{\beta}}
$$

The terms in Eq. (22) are given in Table II where $K_{D}, A, \beta, C_{\max }, r_{b}$, and $r$ are parameters associated with the sorption models. The adsorption term is included in the tracer solution by replacing the $A_{c}$ term, Eq. (19), with the new term

$$
A_{c}=\phi\left(S_{v} \rho_{v} C_{v}+S_{\ell} \rho_{\ell} C_{\ell}\right)+\rho_{r} C_{R}
$$

Chemical reactions are modeled by modifying the source term, $q_{c}$ in Eq. (18), with the addition of the term $q_{c R}$ given below:

$$
q_{c R}=\frac{a_{1} C_{\ell}^{a^{2}}}{a_{3}+a_{4} C_{\ell}}
$$


TABLE II. Sorption Isotherm Models

\begin{tabular}{lcccc} 
Model & Expression & $\alpha_{1}$ & $\alpha_{2}$ & $\beta$ \\
\hline Linear & $C_{R}=K_{D} C_{\ell}$ & $K_{D}$ & 0 & 1
\end{tabular}

Freundlich $\quad C_{R}=A C_{\ell}^{\beta} \quad A \quad 0 \quad 0<\beta<1$

Modified $\quad \frac{C_{R}}{C_{\max }-C_{R}}=A C_{\ell}^{\beta} \quad C_{\max } \quad A \quad 0<\beta<1$

Freundlich

Langmuir $\quad C_{R}=\frac{r_{b} C_{\ell}}{1+r C_{\ell}} \quad r_{b} \quad r \quad 1$

where the quantities $a_{1}, a_{2}, a_{3}$, and $a_{4}$ are user inputted parameters. The general case given in Eq. (24) can sometimes be replaced by a simplier expression $q_{c R}=a_{1}\left(C_{\ell}-a_{2}\right)$. Both of the models are addressed in the input section.

Several well known relative permeability functions are available to the user. They are the simple linear functions, the Corey (1954) relationships, and the Sandia functions (van Genuchten, 1980).

The linear functions are given by

$$
\begin{array}{ll}
R_{\ell}=0 & , S_{\ell} \leq r p_{1} \\
R_{\ell}=\left(S_{\ell}-r p_{1}\right) /\left(r p_{3}-r p_{1}\right) & , r p_{1}<S_{\ell}<r p_{3} \\
R_{\ell}=1 & , S_{\ell} \geq r p_{3} \\
R_{v}=0 & , S_{v} \leq r p_{2} \\
R_{v}=\left(S_{v}-r p_{2}\right) /\left(r p_{4}-r p_{2}\right) & , r p_{2}<S_{v}<r p_{4} \\
R_{v}=1 & , S_{v} \geq r p_{4}
\end{array}
$$

where $r p_{1}, r p_{2}, r p_{3}$ and $r p_{4}$ are user supplied quantities.

The Corey relative permeability functions are given by

$$
\begin{aligned}
& R_{\ell}=\hat{S}_{\ell}^{4} \\
& R_{v}=\left(1-\hat{S}_{\ell}\right)^{2}\left(1-\hat{S}_{\ell}^{2}\right)
\end{aligned}
$$

where $\hat{S}_{\ell}=\left(S_{\ell}-r p_{1}-r p_{2}\right) /\left(1-r p_{1}-r p_{2}\right)$ and where the input quantities $r p_{1}$ and $r p_{2}$ are the residual liquid and vapor saturations respectively. 
The van Genuchten (van Genuchten, 1980) functions are described by the following formulae:

$$
\begin{array}{rlrl}
\hat{S} & =\frac{\left(S_{\ell}-r p_{2}\right)}{\left(r p_{3}-r p_{2}\right)} & \\
R_{\ell} & =\sqrt{\hat{S}}\left[1.0-\left(1.0-(\hat{S})^{1 / \lambda}\right)^{\lambda}\right]^{2} & & , \hat{S}_{\ell}<r p_{3} \\
& =1.0 & & , \hat{S}_{\ell} \geq r p_{3} \\
R_{v} & =1.0-R_{\ell} & &
\end{array}
$$

where $\lambda=r p_{1}$ and $r p_{1}, r p_{2}$ and $r p_{3}$ are parameters supplied by the user.

Composite relative permeability curves, as described by Klavetter and Peters (1986), are also available. See Section III B, macro-command $R L P$ for an input description.

$R_{\ell}$ and $R_{v}$ are restricted by the relationships

$$
\begin{aligned}
& 0 \leq R_{v} \leq 1.0 \\
& 0 \leq R_{\ell} \leq 1.0
\end{aligned}
$$

and the relative permeability functions are truncated to the appropriate value if the above conditions are violated.

The capillary functions included with FEHMN are the linear function and the Sandia capillary pressure model. Our terminology follows that of Pruess (1987).

The linear capillary function model is given by the following equations

$$
\begin{aligned}
& P_{\text {cap }}=c p_{1}, \quad S_{\ell} \leq c p_{2} \\
& P_{\text {cap }}=0.0, \quad S_{\ell} \geq c p_{3} \\
& P_{\text {cap }}=c p_{1} \frac{c p_{3}-S_{\ell}}{c p_{3}-c p_{1}}, \quad c p_{2}<S_{\ell}<c p_{3}
\end{aligned}
$$

where $c p_{1}, c p_{2}$, and $c p_{3}$ are parameters supplied by the user. The restriction $c p_{3}>c p_{2}$ is also necessary.

The van Genuchten functions (van Genuchten, 1980) are described by the following equations

$$
\begin{aligned}
\hat{S} & =\frac{S_{\ell}-S_{\ell r}}{S_{\ell s}-S_{\ell r}} \\
P_{c a p} & =0, \quad S_{\ell} \geq c p_{5} \\
P_{c a p 1} & =P_{o}\left\{(\hat{S})^{-1 / \lambda}-1.0\right\}^{1.0-\lambda} \\
P_{c a p} & =P_{m a x}, P_{c a p 1} \geq P_{m a x} \\
P_{c a p} & =P_{c a p 1}, P_{c a p 1}<P_{m a x}
\end{aligned}
$$

where $\lambda=c p_{1}, P_{o}=1.0 / c p_{3}, P_{\max }=c p_{4}, S_{\ell}=c p_{5}, S_{\ell r}=c p_{2}$. 
The capillary pressure curves approach an infinite value as $S_{\ell}$ approaches 0 . This was observed by Nitao (1988) who suggested using Eq. (30) only to a value of onehalf the maximum suction observed in the rock $\left(P_{\max }\right)$ and linearly extrapolating the capillary pressure to the maximum capillary pressure at $S_{\ell}=0$. We have also employed this technique in FEHMN.

The van Genuchten relative permeability curves and capillary pressure functions are the primary ones used for Yucca Mountain calculations.

If the flow of air is also modeled, appropriate thermodynamic information for air must also be provided. The density of air is assumed to obey the ideal gas law, using atmospheric conditions as our reference state we have

$$
\rho_{a}=1.292864\left(\frac{273.0}{T+273.0}\right)\left(\frac{P}{0.101325}\right)
$$

where $\rho_{a}$ has the units $\mathrm{kg} / \mathrm{m}^{3}, T$ is in ${ }^{\circ} \mathrm{C}$, and $P$ is in $M P a$. The mixture density is given by

$$
\rho_{v}=\rho_{v, w}+\rho_{a}
$$

where $\rho_{v, w}$ is the density of the water vapor. The density of the liquid phase is assumed to be unaffected by the amount of dissolved air it contains.

The enthalpy of air is a function of temperature only

$$
\begin{aligned}
C_{p a} & =6122.0-11.76\left(T^{*}\right)+0.0177\left(T^{*}\right)^{2} \\
h_{a} & =C_{p a}\left(T \cdot 10^{-6}\right)
\end{aligned}
$$

where $h_{a}$ is the enthalpy of air (MJ/kg), $C_{p a}$ is the heat capacity of air (MJ $/ \mathrm{kg}^{\circ} \mathrm{C}$ ), $3 \mathrm{sin}$ $T^{*}=T+273$. The mixture enthalpy for the vapor phase is

$$
h_{v}=h_{v, w}\left(1-\eta_{v}\right)+h_{a} \eta_{v}
$$

where $h_{v, w}$ is the enthalpy of steam and $\eta_{v}$ is the fraction by mass of air in the vapor phase. The enthalpy of the liquid phase is given by the enthalpy of the dissolved air, the heat of solution, and the enthalpy of the water. At present, we neglect the heat of solution and use the enthalpy of air described above:

$$
h_{\ell}=h_{\ell, w}\left(1-\eta_{\ell}\right)+h_{a} \eta_{\ell}
$$


where $h_{\ell, w}$ is the enthalpy of liquid water and $\eta_{\ell}$ is the mass fraction of $a$ in the liquid phase.

Assuming ideal gas behavior, the mass fraction of air in the vapor phase may be expressed as

$$
\eta_{v}=\frac{\rho_{a}}{\rho_{v}}
$$

the mass fraction of air in the liquid phase is assumed to obey Henry's law or

$$
\eta_{e}=\alpha P_{a}
$$

where $\alpha$ is the Henry's law constant $\left(\alpha=1.611 \times 10^{-4} P_{a}^{-1}\right)$ for air and $P_{a}$ is the partial pressure of air.

The viscosity of the vapor phase is assumed to be a linear combination of the air viscosity and the water viscosity

$$
\mu_{v}=\mu_{v, w}\left(1-\eta_{v}\right)+\mu_{a} \eta_{v}
$$

where $\iota_{v, w}$ is the steam viscosity and is obtained from steam data. The viscosity of air is assumed constant

$$
\mu_{a}=1.82 \times 10^{-5} c p
$$

The liquid phase viscosity is assumed to be independent of the amount of dissolved air and is obtained from steam table data:

$$
\mu_{\ell}=\mu_{\ell, w}
$$

Often it is necessary to accommodate changes in the rock porosity and permeability due to changes in effective stress caused by temperature and pore fluid pressure changes. A linear and nonlinear model are currently incorporated into the code for this purpose.

The linear pore pressure model for porosity is given by

$$
\phi=\phi_{0}+\left(1-\phi_{0}\right)\left(c_{r}-c_{g}\right)\left(P-P_{c}\right)
$$

where $\phi$ is the porosity at pressure $P, \phi_{o}$ is the initial porosity at pressure $P_{o}, c_{r}$ is the pore volume compressibility of the rock and $c_{g}$ is the compressibility of the matrix grain material.

The nonlinear model for porosity (Gangi, 1978) currently used in the code is given by 


$$
\phi=\phi_{o}\left[1-\left(\frac{P_{c}}{P_{o}}\right)^{m}\right]
$$

and

$$
P_{c}=\sigma-P-\alpha E \Delta T
$$

where $P_{c}$ is the closure stress, $\sigma$ is the in situ stress (assumed isotropic), $\alpha$ is the coefficient of thermal expansion of the rock, $E$ is Young's modulus, $\Delta T$ is the temperature change and $P_{o}$ and $m$ are parameters in the model.

For either case the effect of stress and temperature changes on permeability are modeled with

$$
k=k_{o}\left(\frac{\phi}{\phi_{o}}\right)^{3}
$$

where $k$ is the permeability at porosity $\phi$.

\section{Discretization of the Governing Equations}

The time derivatives in the Eqs. (1-42) are discretized using the standard first order method given by

$$
f\left(t^{n+1}\right)=f\left(t^{n}\right)+\Delta t\left(a f^{\prime}\left(t^{n+1}\right)+(1-a) f^{\prime}\left(t^{n}\right)\right)
$$

where $f\left(t^{n+1}\right)$ is the desired function at time $t^{n+1}, f\left(t^{n}\right)$ is the known value of $f$ at time $t^{n}, \Delta t$ is the time step, $f^{\prime}$ is the derivative of $f$ with respect to time and $a$ is a weighting factor. For $a=1$, the scheme is fully implicit (backward Euler) and for $a=0$, the scheme is fully explicit (forward Euler).

The space derivatives in the governing equations are discretized using the finite element formulation. The finite element equations are generated using the Galerkin formulations. For a detailed presentation of the finite element method the reader is referred to Zienkiewicz (1977). In this method the flow domain, $\Omega$, is assumed to be divided into finite elements; and variables $P, h$ and $T$, along with the accumulation terms $A_{m}$ and $A_{\mathrm{e}}$, are interpolated in each element:

$$
P=[N]\{P\}, h=[N]\{h\}, T=[N]\{T\}, A_{m}=[N]\left\{A_{m}\right\}, \text { and } A_{e}=[N]\left\{A_{e}\right\}
$$

where $[N]$ is the shape function. Here $h$ is used to represent the generalized energy variable; $T$ in single phase water states, $S$ in $z$-phase water states. 
These approximations are introduced in Eqs. (9), (10), and (20), and the Galerkin formulation (described by Zienkiewicz and Parekh, 1970) is applied. The following equations are derived:

$$
\left[T_{m}(P, h)\right]\{P\}+\hat{C} \frac{\partial A_{m}}{\partial t}+\left\{q_{m}\right\}-g\left\{R_{m}\right\}=\left\{F_{m}\right\}
$$

and

$$
\left[T_{e}(P, h)\right]\{P\}+[K]\{T\}+\hat{C} \frac{\partial A_{e}}{\partial t}+\left\{q_{e}\right\}-g\left\{R_{e}\right\}=\left\{F_{e}\right\}
$$

where

$$
\begin{aligned}
T_{m i j} & =\int_{\Omega} \bar{\nabla} N_{i} \cdot D_{m}^{U P} \bar{\nabla} N_{j} d V, \\
T_{e i j} & =\int_{\Omega} \bar{\nabla} N_{i} \cdot D_{e}^{U P} \bar{\nabla} N_{j} d V, \\
K_{i j} & =\int_{\Omega} \bar{\nabla} N_{i} \cdot \bar{\nabla} N_{j} d V, \\
\hat{C}_{i j} & =\int_{\Omega j} N_{i} N_{j} d V, \\
R_{m i} & =\int_{\Omega j} \frac{\partial N_{i}}{\partial y} N_{j} D_{m}^{U P} \rho_{m} d V,
\end{aligned}
$$

and

$$
R_{e i}=\int_{\Omega j} \frac{\partial N_{i}}{\partial y} N_{j} D_{e}^{U P} \rho_{e} d V
$$

Equations (50) and (51) need some comment. The term $D_{e}^{U P}$ indicates an upstreamweighted transmissibility (Dalen, 1979). This technique has worked well in the low-order elements (3-node triangle, 4-node quadrilateral), where the schemes resemble difference techniques. The upstream weighting is determined by comparing the velocities at the nodes $i$ and $j$. The shape function coefficients are generated in a unique way that requires the integrations in Eqs. (50) and (51) to be performed only once and the nonlinear coefficients to be separated from this integration. The reader is referred to Zyvoloski (1983) for more details.

The integration scheme used in this report is similar to that described by Young (1981). His implementation differs from common methods in that it uses Lobatto instead of Gauss integration. The net effect is that, while retaining the same order of integration accuracy (at least for linear and quadratic elements), there are considerably fewer nonzero terms in the resulting matrix equations. Figure 1 shows a comparison of the nodal connections for Lobatto and Gauss integration methods. It should be noted that these results hold on an orthogonal grid only. If a nonorthogonal grid were introduced, then additional 


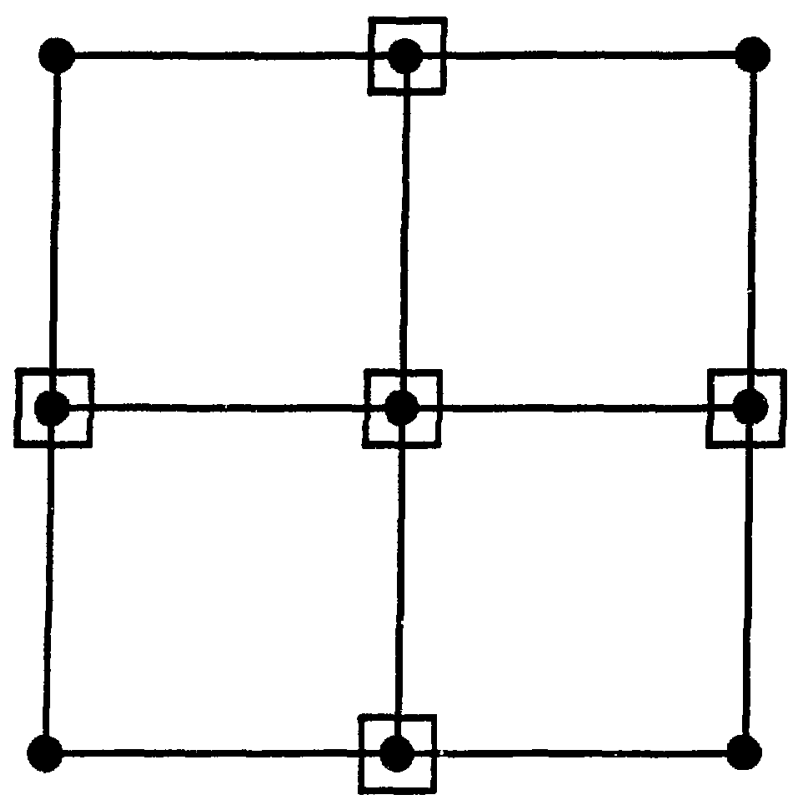

Fig. 1. Comparison of nodal connections for conventional and Lobatto integrations for an orthogonal grid.

nonzero terms would appear in the Lobatto quadrature method. Note also that the linear elements yield the standard 5- or 7-point difference scheme. The reader is referred to Young's paper for more details.

A similar approach is used to solve the transport equation. Following the discussion above the species concentration, $C$, and the species accumulation term, $A_{c}$, are interpolated in each element.

$$
C=[N]^{T}\{C\}, \quad A_{c}=[N]^{T}\left\{A_{c}\right\}
$$

Using these approximators and using a Galerkin approach, we obtain the following equation

$$
\left[T_{c}(C)\right]\{P\}+\left[D_{c}\right]\{C\}+\hat{C} \frac{\partial A_{c}}{\partial t}+\left\{q_{c}\right\}+\left\{R_{c}\right\}=\left\{F_{c}\right\}
$$

when 


$$
\begin{aligned}
T_{c i j} & =\int_{\Omega} \bar{\nabla} N_{i} \cdot D_{m} C_{i j}^{U P} \bar{\nabla} N_{j} d v \\
D_{c i j} & =\int_{\Omega} \bar{\nabla} N_{i} \cdot D_{c} \bar{\nabla} N_{j} d v \\
R_{c i j} & =\int_{\Omega} \frac{\partial N_{i}}{\partial y} \cdot D_{m} C_{i j}^{U P} \bar{\nabla} N_{j} d v
\end{aligned}
$$

where $C_{i j}$ was defined previously and $C_{i j}^{U P}$ is an upstream weighted species concentration. This approach is similar to the finite difference method for solving the transport equations.

\section{Boundary Conditions}

Two mass flow boundary conditions are allowed in FEHMN. They are no flow and specified variable values. The no flow conditions are automatically satisfied by the finite element mesh. Specified variable quantities are obtained in the following manner. A. pressure dependent flow term is used:

$$
q_{i}=I_{i}\left(P_{f l o w, i}-P_{i}\right)
$$

where $P_{i}$ is the pressure at node $i, q_{i}$ is the flow rate, $I_{i}$ is the impedance, and $F_{\text {flow, } i}$ is the specified flowing pressure at node $i$. By specifying a large $I_{i}$, we can force the pressure to be equal to $P_{f l o w, i}$. The energy (or temperature) specified at node $i$ refers only to the incoming fluid value, if fluid flows out of the reservoir, stability dictates that the energy of the in-place fluid be used in the calculations. Species concentration is handled in a manner analogous to the energy variable. Only the incoming species concentration can be specified; if the flow is out of the reservoir the in-place species concentration is used.

In addition to the mass flow boundary conditions, heat flow boundary conditions are also provided. A specified heat flow can be input or a specified temperature obtained

$$
q_{e, i}=I_{e, i}\left(T_{f l o w, i}-T_{i}\right)
$$

where $T_{i}$ is the nodal temperature, $T_{f l o w, i}$ is the specified flowing temperature at node $i, I_{e, i}$ is the impedance to heat flow at node $i$, and $q_{e, i}$ is the heat flow at node $i$. This heat flow is superimposed on any existing heat flow from the other boundary conditions or source terms.

\section{E. Solution Methods}

The application of the discretization methods to the governing partial differential equations yields a system of nonlinear algebraic equations. To solve these equations, the Newton-Raphson iterative procedure is used. This is an iterative procedure that makes use of the derivative information to obtain an updated solution from an initial guess. Let the set equations to be solved be given by 


$$
\bar{F}(\bar{x})=0
$$

where $\bar{x}$ is the vector of unknown values of the variables that satisfy the above equation. The procedure is started by making an initial guess at the solution, say $\bar{x}^{\circ}$. This is usually taken as the solution from the previous time step. Denoting the value of $\bar{x}$ at the kth iteration by $\bar{x}^{k}$, the updating procedure is given by

$$
\bar{x}^{(k+1)}=\bar{x}^{k}-\bar{F}\left(x^{k}\right)\left(\frac{\partial \bar{F}}{\partial \bar{x}^{k}}\right)^{-1}
$$

At each step, the residuals $\bar{F}\left(x^{k}\right)$ are compared with a prescribed error tolerance. The prescribed error tolerance, $\epsilon$, is an input parameter and an $\ell^{2}$ norm is used:

$$
\|F\|_{k}=\left(\sum_{i} F_{i}^{2}\right)_{k}^{1 / 2}
$$

Convergence is achieved when

$$
\|F\|_{k} \leq \epsilon\|F\|_{0}
$$

$\epsilon$ is usually in the range $10^{-4}-10^{-7}$. Semiautomatic timestep control is designed based on the convergence of the Newton iterations. If the code is unable to find a solution $\bar{x}^{k}$ such that the residuals become less than the tolerance within a given number of iterations, the time step is reduced and the procedure repeated. On the other hand, if convergence is rapid, the timestep is increased by multiplying with a user supplied factor, thus aliowing for large timesteps when possible.

The matrix equation to be solved at each Newton-Raphson iteration has the form

$$
[A]\{x\}=\{b\},
$$

where $[A]$ is the Jacobian matrix, $\{x\}$ is the solution vector, and $\{b\}$ is the residual. The $[A]$ matrix is sparse and banded. For computation, $\{x\}$ is replaced by $\{\delta x\}=\{x\}^{n+1}-\{x\}^{n}$, and $\{b\}$ is replaced by $\{r\}=\{b\}-[A]\{x\}^{n}$, where $n$ is the iteration number. (Note that this is different from the overall Newton iteration.) Thus, only changes in $\{x\}$ are calculated.

Complete Gaussian elimination or factorization on Eq. (59) would result in fill-in of all the bands between the lowest subdiagonal and the highest superdiagonal bands. Incomplete factorization involves finding only approximate upper and lower triangular matrices $\widetilde{L}$ and $\widetilde{U}$, requiring very little fill-in. The computing effort for obtaining the incomplete factors is much less than for the complete factors. However, an iterative procedure is now 
required. The presentation of the theory below follows that of Saad and Schultz (1986). The matrix $[A]$ is partially factored into upper and lower triangular form

$$
[\bar{A}]=[\widetilde{L}][\widetilde{U}],
$$

where $[\bar{A}] \neq[A]$ because, in general, $[\widetilde{L}]$ and $[\widetilde{U}]$ will contain only some of the steps of the elimination process. To incompletely factor a matrix, a prior knowledge of the fill-in pattern is required. A symbolic factorization is performed (just once) to establish where the fill-in occurs and to determine the order of each term. Figure 2 shows a matrix and its first level of fill (0's) resulting from factorization of the original matrix elements (x's). We could continue the process to get higher-order terms, but usually the lower-order terms are all that are necessary (Zyvoloski, 1986). The amount of factorization is limited by computer storage considerations. Once factored, the solution is easy to carry out as it consists of a forward and a backward substitution:

$$
\{v\}=[\widetilde{U}]^{-1}\left\{[\widetilde{L}]^{-1}\{r\}\right\}
$$

where $\{v\}$ is the approximate change in $\{x\}$. The following recursion scheme is used to refine the approximation:

$$
\begin{aligned}
& \{v\}^{n+1}=[\bar{A}]^{-1}\{r\}^{n}=[\widetilde{U}]^{-1}\left\{[\widetilde{L}]^{-1}\{r\}^{n}\right\} \\
& \{x\}^{n+1}=\{x\}^{n}+\{v\}^{n+1} \\
& \{r\}^{n+1}=\{r\}^{n}-[\bar{A}]\{v\}^{n+1}
\end{aligned}
$$

where $n$ is the iteration index and $\{x\}^{0}=0$ since the original matrix equation is written in residual form [see paragraph after Eq. (59)]. The solution scheme, comprising Eqs. (61)-(64), may be accelerated by over-relaxation procedures, but it is better to use orthogonalization-minimization. If the Jacobian matrix were symmetric, conjugate gradient could be used. For nonsymmetric matrices, the GMRES (Generalized Minimal Residual Equation Solver) procedure (Saad and Schultz, 1986) is used.

For comparison purposes, the simple over-relaxation procedure is discussed first. In the recursion scheme [Eqs. (62)-(64)], Eq. (63) is replaced by $\{x\}^{n+1}=\{x\}^{n}+w\{v\}^{n+1}$, and Eq. (64) is replaced by $\{r\}^{n+1}=\{r\}^{n}-w[A]\{v\}^{n+1}$. Here $w$ is the over-relaxation parameter and satisfies the relation $1<w<2$. After Eq. (64) is calculated, the procedure returns to Eq. (61) and continues until $\{r\}^{n+1}$ is reduced to some predetermined value. For many reservoir problems, convergence is extremely slow, and the ortho-minimization procedures provide a valuable alternative. 


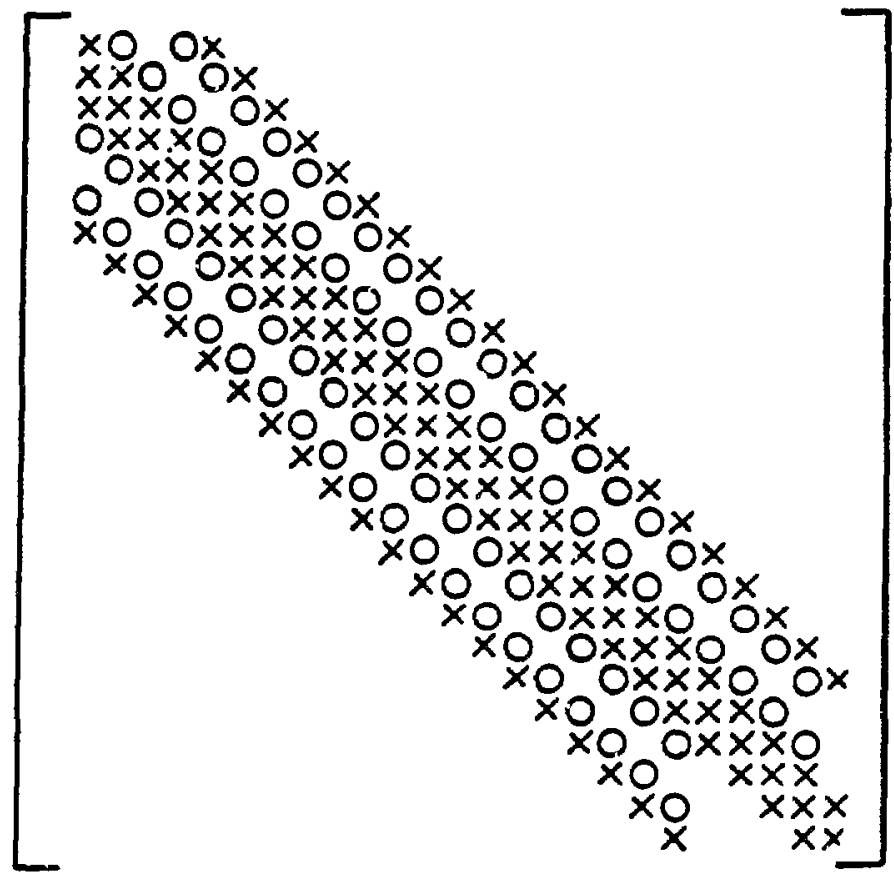

Fig. 2. Matrix band structures showing second-order fill-in.

$x$ - original matrix positions.

o - fill-in with order 2 factorization.

The GMRES algorithm is

1. Set $\{r\}^{\circ}=\{b\}$ and set $\{v\}^{1}=\{r\}^{\circ} /\left\|r^{\circ}\right\|$ where $\|r\|$ is the sum squared norm $\left(\|r\|=\left\{\sum_{i=1}^{n} r_{i}\right\}^{1 / 2}\right)$ of $\{r\}$ and $n$ is the number of unknowns. We also assume that $\{x\}^{\circ}=\{0\}$.

2. Perform orthogonalization calculation, $m=1$, NORTH

$$
\begin{aligned}
\{w\}^{m+1} & =[A]\{v\}^{m}-\sum_{j=1}^{m}\left([A]\{v\}^{i},\{v\}^{i}\right)\{v\}^{i} \\
h_{m}^{m+1} & =\left\|\{w\}^{m+1}\right\| \\
\{v\}^{m+1} & =\{w\}^{m+1} / h_{m}^{m+1}
\end{aligned}
$$

Where NORTH is the maximum number of orthogonalizations allowed.

3. Form approximate solution $(n=N O R T H)$

$$
\{x\}^{m}=\{x\}^{\circ}+[V]^{m}\{y\}^{m}
$$


where $[V]^{m}$ is an $n$ by $m$ matrix which contains the $\{v\}^{i}(\mathrm{i}=1, m)$ and $\{y\}^{m}$ is an $n$ dimensional vector which minimizes the function.

$$
f(y)=\left\|B\{e\}^{1}-[H]^{m}\{y\}\right\| .
$$

Here $B=\left\|r^{\circ}\right\|,\{e\}^{1}$ is the unit vector, and $[H]^{m}$ is a $k+1(=\mathrm{n})$ by $k$ matrix whose only nonzero entries are the $h_{i}^{i+1}$ calculated in Eq. (66). This minimization is economically done using $Q-R$ factorization. (See Saad and Shultz, 1986, for details.)

4. Calculate $\left\|r_{m}\right\|$ where

$$
\{r\}^{m}=\{b\}-[A]\{x\}^{m}
$$

If $\left\|r_{m}\right\| \leq \epsilon$, some specified tolerance, then the process is complete. Otherwise we set $\{x\}^{\circ}=\{x\}^{m},\{v\}^{1}=\{r\}^{m} /\left\|r^{m}\right\|$ and proceed from Eq. (65) of the GMRES algorithm. There is an intimate relationship between the outer iteration (NewtonRaphson) and the inner iteration (solution of linear equations). If the outer iteration is far from convergence, we can solve the linear system of equations with a rather large tolerance. As the outer iterations converge we must likewise have a finer tolerance for the inner iteration. The method used here is one developed by Diaz, Jines, and Steihaug (1985). Regions of linear and quadratic convergence are defined, and multipliers for the square of the residuals and the residuals are specified. Comparing the magnitude of the terms determines the largest possible tolerance for the inner iteration.

A mathematical theory similar to that for conjugate gradient methods (for example, showing a reduction in condition number witb each iteration) is not available. In basic termis the $h_{m}^{m+1}$ in Eqs. (66) and (67) ensure that the $\{v\}^{i}$ are orthogonal and that the $\{y\}^{m}$ in Eqs. (68) and (69) are such that the square of the $\{r\}^{n+1}$ is minimized. For more details the reader is referred to Meijerink and Van der Vorst (1981), Behie and Forsythe (1984), and Behie and Vinsome (1982). To minimize the storage required, the procedure is restarted after every NORTH iteration. The more orthogonalizations before restarting, the faster the convergence, but this benefit must be weighed against the greater storage and longer running time required for the additional orthogonalizations.

\section{F. Dual Porosity Formulation}

Many problems are dominated by fracture flow. In these cases the fracture permeability controls the pressure communication in the reservoir even though local storage around the fracture may be dominated by the porous rock which communicates only with the closest fractures. Moench (1984) has studied several wells in the saturated zone beneath Yucca Mountain and found the results could be understood if dual porosity methods were used. Figure 3 depicts the dual porosity concept. The computational volume in the figure 
consists of a fracture which communicates with fractures in other computational cells, and matrix material which only communicates with the fracture in its computational cells. This behavior of the matrix material is both a physical limitation and a computational tool. The physical limitation results from the model's inability to allow the matrix materials in different cells to communicate directly. This yields only minor errors in saturated zone calculations, but could pose larger errors in the unsaturated zone where capillary pressures would force significant flow to occur in the matrix material. The computational advantages will be addressed at the end of this section.

\section{matrix material}

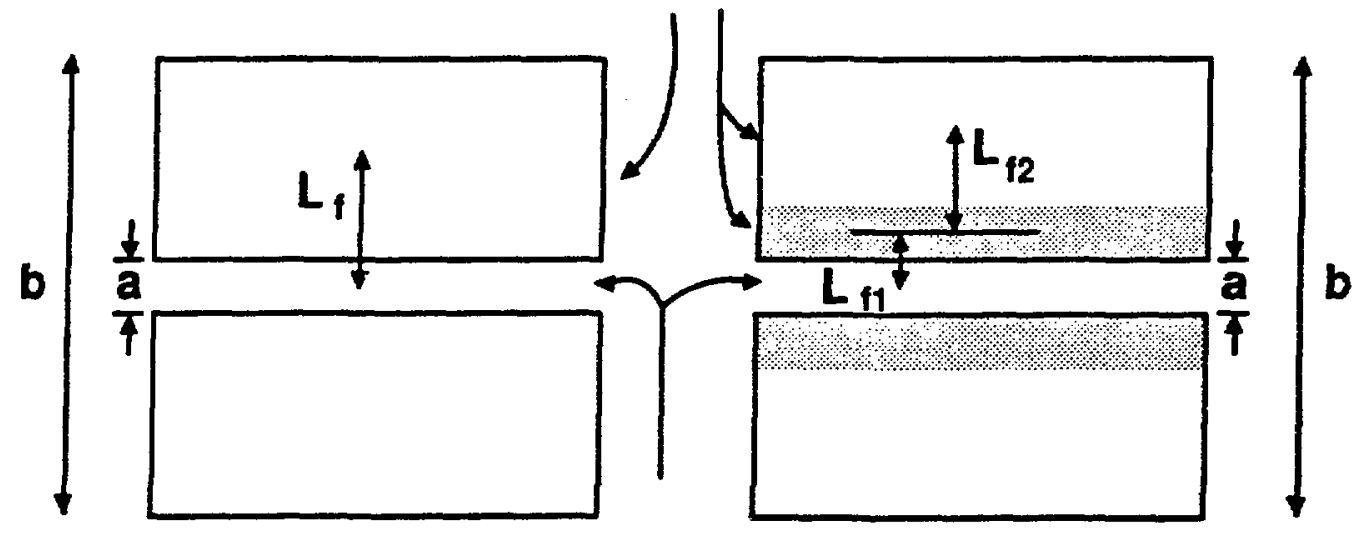

fracture

\section{a) One matrix node}

b) Two matrix nodes (FEHMN)

Fig. 3. Computational volume element showing dual porosity parameters.

Two parameters characterize a dual porosity reservoir. The first is the volume fraction, $V_{f}$, of the fractures in the computational cell. For the simple system shown in Fig. 3a this fraction is $a / b$. The second parameter is related to the fracture's ability to communicate with the local matrix material. In the literature this parameter takes a variety of forms. The simplest is a length scale, $L_{f}$. This quantifies the average distance the matrix material is from the fracture. This length scale is also shown in Fig. 3a. With just one node in the matrix material the transient behavior in the matrix material cannot be modeled. To improve this situation, two nodes are used in FEHMN to represent the matrix material. Conceptually, this is the same formulation as just described with only additional fracture volume needed (it is assumed the length scale of each matrix volume is proportional to the volume fraction). This is shown in Fig. $3 \mathrm{~b}$. More matrix nodes could be added, but data is rarely good enough to justify the use of even two matrix nodes. We note here that the 
simple slab model depicted in Fig. 3 is just one of several different geometric arrangements. Moench (1984) and Warren and Root (1963) list other reservoir types. All of them are similar in the assumption of a local one dimension connection of the matrix to the fracture.

Computationally, the volume fraction and length scale are used to create one dimensional versions of Eq. (9), Eq. (10), Eq. (16), and Eq. (20). The length scale is used to modify spacial difference terms and the volume factors are used to modify the accumulation terms [the $\hat{C}$ matrix in Eq. (44)]. The volume fractions for FEHMN are given by

$V_{f}=1-V_{f 1}-V_{f 2}$, volume fraction of fractures

$V_{f 1}$ - fraction of first matrix volume (input)

$V_{f 2}$ - fraction of second matrix volume (input)

the length scales are given by

$L_{f}=L_{f 0} V_{f}$, length scale for the fracture volume

$L_{f 0}$ - length scale (input)

$L_{f 1}=L_{f 0} V_{f 1}$ length scale of first matrix volume

$L_{f 2}-L_{f 0} V_{f 2}$, length scale of second matrix volume

The geometric factor representing the spacial differencing of the one dimensional equation for flow between the fracture and the first matrix node [analogous to the geometric part of Eq. (46) and Eq. (47)] is given by

$$
T_{f f 1}=\frac{1.0}{L_{f 1}}\left(L_{f 1}+L_{f}\right)
$$

The analogous term for the flow from the first matrix volume to the second matrix volume is given by

$$
T_{f 1 f 2}=\frac{1.0}{L_{f 2}}\left(L_{f 1}+L_{f 2}\right)
$$

The one dimensional nature of the equations provides a computationally efficient method to solve the algebraic equations arising from the dual porosity simulation. Equation (73) shows the matrix equation arising from a dual porosity simulation.

$$
\left[\begin{array}{lll}
A_{00} & A_{01} & A_{02} \\
A_{10} & A_{11} & A_{12} \\
A_{20} & A_{21} & A_{22}
\end{array}\right]\left\{\begin{array}{l}
P_{0} \\
P_{1} \\
P_{2}
\end{array}\right\}=-\left\{\begin{array}{l}
R_{0} \\
R_{1} \\
R_{2}
\end{array}\right\}
$$

Here the subscript 0 refers to the fracture, 1 refers to the first matrix volume, and 2 refers to the second matrix volume.

The $P$ represents the unknown variable or variable pair. The one dimensional characte. of the matrix diffusion means that the second matrix node can only depend on the first 
matrix node. Therefore, the submatrix $A_{20}$ is empty. The fact that matrix nodes cannot communicate with matrix nodes in other computational cells means that the submatrices $A_{21}$ and $A_{22}$ are diagonal. Therefore we may write

$$
\left\{P_{2}\right\}=\left[A_{22}\right]^{-1}\left[-\left\{R_{2}\right\}-\left[A_{21}\right]\left\{P_{1}\right\}\right]
$$

where the inversion is trivial because $\left[A_{22}\right]$ is diagonal. Substituting this expression into the equation for the first matrix node we have

$$
\left[A_{10}\right]\left\{P_{0}\right\}+\left[A_{11}\right]\left\{P_{1}\right\}+\left[A_{12}\right]\left[A_{22}\right]^{-1}\left\{-\left\{R_{2}\right\}-\left[A_{21}\right]\left\{P_{1}\right\}\right\}=-\left\{R_{1}\right\}
$$

Rearranging, we have

or

$$
\left[A_{10}\right]\left\{P_{0}\right\}+\left[\left[A_{11}\right]-\left[A_{12}\right]\left[A_{22}\right]^{-1}\left[A_{21}\right]\right]\left\{P_{1}\right\}=-\left\{R_{1}\right\}+\left[A_{12}\right]\left[A_{22}\right]^{-1}\left\{R_{2}\right\}
$$

$$
\left\{P_{1}\right\}=\left[\widetilde{A}_{11}\right]^{-1}\left[\left\{\widetilde{R}_{1}\right\}-\left[A_{10}\right]\left\{P_{0}\right\}\right]
$$

where

$$
\left[\widetilde{A}_{11}\right]=\left[A_{11}\right]-\left[A_{12}\right]\left[A_{22}\right]^{-1}\left[A_{21}\right]
$$

and

$$
\left\{\widetilde{R}_{1}\right\}=-\left\{R_{1}\right\}+\left[A_{12}\right]\left[A_{22}\right]^{-1}\left\{R_{2}\right\}
$$

where the inversion and multiplications are trivial because of the diagonal nature of the matrices involved. Equation (76) may next be substituted into the equation for the fracture variables. Noting that $\left[A_{02}\right]$ is empty (the fracture can only communicate with the first matrix volume) we have

$$
\left[A_{00}\right]\left\{P_{0}\right\}+\left[A_{01}\right]\left[\widetilde{A}_{11}\right]^{-1}\left\{\left\{\widetilde{R}_{1}\right\}-\left[A_{10}\right]\left\{P_{0}\right\}\right\}=-\left\{R_{0}\right\}
$$

Rearranging terms we have

$$
\left[\left[A_{00}\right]-\left[A_{01}\right]\left[\tilde{A}_{11}\right]^{-1}\left[A_{10}\right]\right]\left\{P_{0}\right\}=-\left\{R_{0}\right\}+\left[A_{01}\right]\left[\tilde{A}_{11}\right]^{-1}\left\{\widetilde{R}_{1}\right\}
$$

Equation (78) consists of an augmented fracture matrix of the same form as the original fracture matrix $\left[A_{00}\right]$. The operations carried out only add a few percent to the solution time required to solve a single porosity system. After the solution of Eq. (78) is obtained with the methods described in Section $E$, the solution in the fracture volume can be obtained by using Eq. (74) and Eq. (76). 


\section{CODE DESCRIPTION}

In this section we present three important descriptions of FEHMN. First descriptions of all the subroutines are presented. Next the common block variables and calling parameters are described. Finally, the subroutine call tree structure for FEHMN is provided.

\section{A. Subroutines}

The main program of the code FEHM is GZ. This program calls subroutine DATA to initialize most of the common bluck variables and calls subroutine INPUT which initializes most non-array variables and directs the data input. GZ also performs one time startup calculations: scaling of some variables, directing the calculation of element coefficient arrays, and the setting up of equation solution schemes. The major time step loop is contained within GZ. In this loop the model equations are solved at each time step, solution parameters adjusted, and variables updated for the next time step. Plotting data is also output from this loop.

The following subroutines are called from GZ (excluding calls to intrinsic functions): ANONP, BNSWER, COEFFC, CONCEN, CONTR, CO2CTR, DATA, DATCHK, DISK, DUAL, ENTHP, INPUT, NEAR3, PEINT, PLOT, RADIUS, RARNG, RESETV, SETORD, SICE, SLVESU, STEADY, STORSX, STRESS, TIMCRL, TYMING, USER, VARCHK, VELOC, WELBOR, WRTOUT.

Table III contains a complete aiphabetical listing of the FEHMN subroutines. A brief description of each routine is provided along with listings of which subroutines call the-given routine and which subroutines it calls (intrinsic functions are excluded).

TABLE III. Subroutine Calls Within FEHMN

Name Description

Calls

Called by

ANONP Categorize elements. Call routines GENCOF to generate finite element coefficients.

BNSWER

Call routines to assemble finite ele-

VARCHK, OUTBND, ment equations and solve for the Newton-Raphson equations. GENSL13, GENSL14, GENSL1

GZ

Calculate capillary pressure functions.

CNSWER Call routine to generate tracer trans- THERMC, GENCON port equations. Call tracer equation of state routines.

INPUT, THRMWC, THERMW

GZ, THRMWC

\section{CSOLVE}


TABLE III. Subroutine Calls Within FEHMN

Name

Description

Calls

Called by

COEFFC Change coefficients of polynomial

GZ

fits of the thermodynamic proper-

ties specified.

CONCEN Organize calls required to run tracer transport model.

RDCON, CSOLVE, WRTCON, PLOTC, DISKC, CONTRC

PSAT

CO2CTR Read input for noncondensible gas and initialize variables associated with the noncondensible gas.

CSOLVE Organize tracer solution so smaller time steps can be used for the tracer solution than for the flow solution.

DATA Initialize cornmon block variz,bles.

CNSWER, PLOTC1 THERMC

CONCEN, VELOC at particular nodes.

CONTRC Write out tracer data for contour plots.

DATCHK Initial value analysis and data check.

DISK

Read and/or write to files for restart purposes.

DISKC Read and write restart files for tracer variables.

DUAL

Read dual porosity data. Initialize dual porosity variables.

VARCHK, DUALFA, DUALEX

CONCEN, STRESS

GZ,TIMCRL

CONCEN

GZ, TIMCRL, GENSL4, GENSL1, INPUT DUAL

DUALEX Extract dual porosity solution from

GZ, TIMCRL, WRTOUT, INPUT CONCEN

GZ

GZ CONTR
GENCON CONTR
GENCON

GZ, TIMCRL

\section{CONCEN}

WRTOUT, DISK, INPUT,

CONEQ1 Generate the equations of tracer transprimary variable solution. 
DUALFA Assemble and load dual porosity VARCHK, GENEQ1, DUAL solution and modify primary vari- GENEQ3 ables solution to account for dual porosity effects.

ENTHP Calculate the temperature for a given input enthalpy.

GZ, STEADY

GENCOF Call routines to generate finite eleGNCF3, GNCF2 ANONP ment coefficients. Perform the numerical integration of the elements.

GENCON Call routines to generate tracer CONEQ1, SOLVE, equations. Call solver to obtain RD1DOF Newton-Raphson equations for tracer variables.

GENDAT Generate coordinates and element INPUT, ZONE information in simple geometric problems.

GENEQC Generate equations for Newton-Raphson GENSL4 corrections for the water and noncondensible gas flow.

GENEQ1 Generate equations for Newton-Raphson corrections for water/vapor flow with no noncondensible gas.

GENEQ3 Generate equations for Newton-Raphson corrections for heat conduction only (i.e., permeability).

CNSWER

GENSI4

DUALFA, GENSL1

DUALFA, GERSL4, GENSL1, GENSL3

GENSL1 Call routines to generate the Newton-Raphson equations for water only problems. Call equation GENEQ1, GENEQ3, BNSWER solver subroutine.

GENSL3 Call routines to generate the Newton-GENEQ3, SOLVE, Raphson equations for heat conduc- RD1DOF

BNSWER tions only. Call equation solver routine.

GENSL4 Calls routines to generate equations for water/noncondensible gas problems and calls equation solver.

GENEQC, GENEQ3, DUAL, NORMAL, SOLVE3, RD3DOF

BNSWER DUAL, NORMAL, SOLVE2, RD2DOF 
Name

GEOIN

GNCF2

GNCF3

INPUT

Initialize variables, read input, write out some input information.

IOFILE Open up initial pressure distribution when gravity is present (enabled).

LUDBKSB Performs forward and rock substitution for $N$ degree of freedom matrix elements.

LUDCOMP Performs Gauss elemination on $N$ degree of freedom matrix elements.

NEAR3

NELMFL

NOPCNR

NOPCNV

NORMAL
Finds nearest node to a set of coordinates.

Load nodal connection array.

Identify form of incomplete LU decomposition matrix for the NewtonRaphson solution matrix with reordering of the node numbering.

Identify form of LU decomposition matrix for the Newton-Raphson solution matrix with no recording of the node numbers.

Normalize Newton-Raphson equations and calculates sum-squared sum of residuals.
Calls

Called by

INPUT,ZONE

SHAP2R

SHAP3R, SHAP3P

GENCOF

GENCOF

CAPPR,CONC CO2CTR,DUA GENDAT,GEC IOFILE,PORO RLPERM, SIC STHER,STRE: USER, WELBO ZONE

INPUT

\section{SOLVEN}

SOLVEN

GZ

GENCOF

SLVESU

SLVESU

GNSL4, GENS 
TABLE III. Subroutine Calls Within FEHMN

Name

Description

Calls

Called by

OUTBND Test the dependent variables to de-

BNSWER termine if they are within the bounds set by the thermodynamics properties.

PEINT Set up initial temperature gradiGZ ents where gradient information is user specified.

PLOT Write out data for time history plots GZ, TIMCRL at particular nodes.

PLOTC1 Write out tracer data for time history plots. Print out at flow time steps.

POROSI Read in data for pressure depen- ROCK dent porosity and permeability models. Calculate porosity and permeability functions.

PSAT Calculate the saturation pressure of water for a given temperature.

CONCEN RDCON

INPUT, WRTOUT, THERMW

RADIUS Modify finite element coefficients to obtain a radial model.

RARNG Rearrange 3-D coordinates to obtain 2-D problems when enabled.

RDCON Read in tracer data. Initialize tracer THEPMC

CONCEN variables.

PLOTC1

Solves the equations generated for SOLVE

GENSL3, heat conduction by a reduced degree of freedom method.

RD2DOF Solve the equations generated for the water only problems by the reduced degree of freedom method.

RD3DOF Solve the equations generated for water/noncondensible gas problems SOLVE

VARCHK, CO2CTR, THRMWC GZ

GZ

RD1DOF by the reduced degree of freedom by the red
method.

SOLVE

GENSL1

GENSL4

SOLVE2, GENCON

\author{
GENL1
}


RESETV Reset the dependent variables to

GZ the last time step value. Used when iteration limits are exceeded and a particular time step is restarted.

RLPERM Calculate relative permeability functions.

GZ, THRMWC, THERMW

SFN2R Interpolation routine used by ZONE (2-D).

SFN3R Interpolation routine used by ZONE (3-D).

SHAP2R Evaluate 2-D finite element shape

GNCF2 functions at quadrature points.

SHAP3P Evaluate 3-D prism elements at quadra-

GNCF3

SHAP3R Evaluate 3-D finite elements shape

GNCF3 functions at quadrature points.

SICE

Reads in data for simulation with STHER

GZ,WRTOUT ice present.

SLVESU

Set up equation solver by identifying fill-in positions in the NewtonRaphson matrix.

SOLVE Solve the one degree of freedom linear system of equation.

INPUT

NOPCNV, NOPCNR, GZ STORAG

RD3DOF, RD2DOF, GENSL3, RD1DOF, GENCON

SOLVEN

SOLVE2
Solve the $N$ degree of freedom linear system of equations.

Solve the two degree of freedom linear system of equations.

\section{LUBKSB}

LUDCMP
RD3DOF, GENSL1 
SOLVE3 Solve the three degree of freedom linear system of equations.

GNSTR3, GENSL4, RD3DOF

\section{STEADY}

STORAG

Set up initial pressure distribution when gravity is present (enabled).

BNSWER, ENTHP

Write out storage requirements of matrices and arrays associated with the solution of linear equations (NewtonRaphson equations).

\section{STORSX}

Manage the storage or retrieval of element coefficients from auxiliary file.

THERMC Evaluate tracer equation of state information.

THERMW Evaluate the thermodynamic properties (density, enthalpy, and viscosity) as a function of pressure and temperature (or saturation).

THRMWC Evaluate the thermodynamic properties (density, enthalpy, and viscosity) as a function of pressure, temperature and partial pressure of noncondensible gas for water/noncondensible gas problems.

TIMCRL Control time step information and stopping criteria.

TYMING Calculate CPU time for a particular computer run.

CAPPR, POROSI

RLPERM

VFCAL

RLPERM CAPPR,

PSAT, VFCAL

GZ

GZ

SLVESU
RDCON, CNSWER VARCHK, SOLVE

VARCHK
DUAL, DISK, CO2CTR, GZ PLOT, CONTR

\section{SYSTEM}

CLOCK*

USER

GZ

GZ,INPUT A user programmed subroutine that variably every time step. Disabled for YMP work.

*Call is made to the system clock of the computer on which it is run. 
USERC User defined subroutine for changing common variables associated with tracer transport. Disabled for YMP project.

VARCHK

Decide, based on current pressure, temperature and saturation values current phase state (fully saturated, partially saturated). Call routine THERMW or THRMWC to update thermodynamic properties of density, enthalpy, and viscosity. Add the Newton-Ralphson corrections to the dependent variables.

VELOC Calculate velocities in coordinate directions.

VFCAL Change porosity and permeability as functions of pressure (when enabled).

WRTCON Write output for tracer at specified intervals.

WRTOUT Print output information at a user specified interval.

THRMWC, THERMW, PSAT

GZ, BNSWER, DUAL, DUALFA

GZ, CONTR

THRMWC, THERMW

CONCEN

POROSI, CO2CTR, CONCEN, SICE,

ZONE
GZ

INPUT
Divide the input problem space by allowing the user to define zones geometrically and then labeling the nodes as to which zone they belong to. These zones are then used to assign properties to the nodes.
GENDAT, SFN3R, SFN2R, GEOIN 


\section{B. Common Block and Parameter Statements}

The common block and parameter statements are contained in INCLUDE files. The INCLUDE files needed to compile FEHMN are COMAI, COMBI, COMCI, COMDI, COMEI, COMFI, COMGI, COMHI, COMII, COMPRI, and DAVIDI. The INCLUDE file COMDTI contain the parameter statements and will be described first. If a variable is entered, the input MACRO statement is indicated parenthetically.

\section{INCLUDE fle COMDTI (parameter statements)}

Only par ameters that are different from those found in COMPRI are described.

No

N2

N3

N4

N5

N6

N7

N8

NR

NBD

LDN

NELMD

connectivity array

NNOP - maximum array space for LU decomposition connectivity array

NN

NQ

NE1

NE2

NE3

NE5

NE6

NE7

NE8

NE9

NE10

NE11

NE12

NE13

NE14

NE15

- maximum number of nodes allowed

- $2 *$ N0, storage parameter

- $3 *$ N0, storage parameter

- array storage parameter for noncondensible gas solution

- array storage parameter for dual porosity solution

- array storage for ice solution

- array storage for tracer solution

- array storage for variable porosity solution

- maximum space allowed for each finite element coefficient array

- $180^{*}$ N0 maximum array space for incomplete LU decomposition matrix

- maximum array space needed for Jacobian array matrix

- maximum number of connected elements

- maximum array space for each finite element coefficient array associated with the stress solution

- array size of common block /FEBB/, NELMD + NNOP + 4NO

- array size of common /FEB/, NO + 9NR + 6NQ

- array size of common block /FBS/, 3NO + 336

- array size of common block / FCC/, 39N0 + 5N7

- array size of common block / FDD/, 36N0

- array size of common block / FDD1/, 14N7 + 870

- array size of common block / FFD2/, 7N8 + 4

- array size of common block / FDDI/, $5 \mathrm{NO}+1$

- array size of common block / FHH/, 14N0

- array size of common block / CO2/, 32N4 + 1

- array size of common block / FGG/, 9NN + 2N3

- array size of common block / DUALP/, 23N5

- array size of common block /FICE/2*N6 +1

- array size of common block/ICE/N6 


\section{INCLUDE File COMAI}

\section{common/FAA/}

IAB - iteration counter in stress routines

IAC - counter for print-out interval

IAD - current iteration number in flow solution

IACCMX - maximum iterations allowed for time step increase (tracer solution)

IAMM - maximum iterations allowed for time step increase (heat and mass solution)

IAMX - iteration count after which the time step will be halved

ICAPP - indicates capillary pressure model

ICE - parameter which indicates if the ICE solution is enabled

ICEN - parameter which indicates if the tracer solutions enabled

ICF - parameter indicating status of tracer solution

ICS - parameter indicating status of tracer solution

ICGTS - parameter controlling the time of solution parameter changes

ICHNG - number of thermodynamic region changes

ICNL - indicates problem dimension

ICO2 - indicates if noncondensible gas solution is enabled (CO2I)

ICONTR - parameter used in contour plot management

IDOF - number of degrees of freedom per node for the current problem

IDUALP - parameter which indicates if the dual porosity solution is enabled (DUAL)

IFINSH - indicates if the finishing criteria for the simulation is achieved

IHF - parameter indicating status of flow solution (TRAC)

IHS - parameter indicating status of flow solution (TRAC)

III - print-out interval, number of time steps (TIME)

IFLAG - flag used in input subroutine

IINP - unit number input file

ILT - parameter used in time step control

INTG - indicates integration type used

IPOROS - indicates if deformation model is enabled (PPOR)

IPQZ - the number of nodes used for the contour plot output

IPSAT - parameter regulating call to subroutine PSAT (EOS)

IOUT - unit number for output file

IREAD - unit number for restart file (to read)

IREORD - parameter to reorder system of linear equations

IRPD - indicates relative permeability model

ISTFS - parameter indicating if the stress solution is enabled

ISAVE - unit number for restart file (to write)

ITER - number of iterations of last call to SOLVE(N) subroutine

ITERT - intermediate iteration counter

ITOTAL - total iteration count

ITSAT - parameter specifying the setting of saturation temperature

IYEAR - current year in simulation 
IVFCAL - indicates if VFCAL subroutine will be called

IW - number of storage locations needed to store geometric input types

IWELB - indicates if wellbore solution is enabled

L - current time step number

LDA - parameter which specifies if the geometric coefficients are saved (CTRL)

M - total number of nodes used for output information (NODE)

MLZ - out of bounds node

MAXIT - maximum number of iterations allowed before time step is halved (CTRL)

MINK - number of active variables

MONTH - current month in simulation

$N \quad$ - total number of nodes

NBND - maximum number of nonzeros per row in the incidence matrix

NCNTR - contour plot interval (CONT)

NDEM - coordinate direction perpendicular to the contour plane

NI - number of integration points per element

NICG - parameter used in time step control

NEI - total number of elements in the problem (ELEM)

NEQ - number of nodes, not including dual porosity nodes (CORD)

NEIGH - maximum number of neighbors occur in tracer solution

NEMX - number of unique (geometrically) elements

NORTH - maximum number of orthogonalizations allowing in (CTRL)

NS - number of nodes per element (ELEM)

NSAVE - indicates if a restart file will be created in the current problem

NSNK - number of source or sink terms

NSTEP - maximum number of time steps

/FAAR/

AIAA

ANO

AM0

AMASS

AMP

ASTEAM

ASTM0

AENER

AW

AWC

AWT

AY

AYC

CONTIM

DAY

DAYCF

DAYCMM

DAYCMX
- time step multiplication factor (CTRL)

- initial tracer concentration (TRAC)

- initial mass in problem

- current mass in problem

- initiai energy in system problem

- current steam mass in problem

- initial stem mass in problem

- current energy in problem

- time step weighting parameter for heat and mass solution (CTRL)

- time step weighting parameter (TRAC)

- value of implicitness factor

- time step weighting parameter for tracer (TRAC)

- time step weighting parameter for tracer

- interval (days) for contour plot output

- current time step size in days

- time at which tracer solution stops (TRAC)

- minimum time step size for tracer solution

- maximum time step size for tracer solution 

DAYCS - time at which tracer solution starts (TRAC)
DAYHF - time at which flow solution stops (TRAC)
DAYHS - time at which flow solution starts (TRAC)
DAYMAX - maximum time step allowed (CTRL)
DAYMIN - minimum time step allowed (CTRL)
DAYNEW - parameter used in time step control
DAYS - current simulation time
DAYSCN - next time for contour plot
DAYSI - simulation time at last time step
DAYSP - time at next time step
DEN - intermediate value of mass accumulation term
DENE - intermediate value of energy accumulation term
DEPCNG - depth at which temperature gradient changes
DIFE - energy balance error
DIFM - mass balance error
DITND - next time step change time
DNWGT - upwind weighting parameter
DNWGTA - upwind weighting parameter in tracer solution
DTOT - current time step size in seconds
DTOTC - tracer time step size in seconds
DTOTDM - last time step size in seconds
EPC - specified solution tolerance for tracer solution
EPE - tolerance for Newton-Raphson iteration
EPS - tolerance for linear equation solver
Fo - initial time step residual
FDUM - current sum squared of residuals
GRAD2 - parameter in description of temperature gradient
GRADNT - parameter used in description of temperature gradient
GRAV - value of gravity
G1 - iteration accuracy control parameter (ITER)
G2 - iteration accuracy control parameter (ITER)
G3 - iteration accuracy control parameter (ITER)
OVERF - over relaxation factor for SOR equations (ITER)
PEIN - initial pressure of problem (INIT)
POW - power output for a given time step
QT - total outflow for time step
QTE - total energy outflow for time step
QTOT - total outflow for problem
QTOTE - total energy outflow for problem
QTOTEI - intermediate energy flow total
QTOTI - intermediate flow total
QUAD - parameter used in temperature gradient
RNMAX - maximum run time allowed
SECMA - maximum time step size in seconds 
STR - multiplier for Newton-Raphson corrections

STRD - multiplier for Newton-Raphson corrections

SV - vapor saturation of a node

SW - liquid saturation of a node

TEOUTF - parameter used in calculation of energy output

TIMS - ending simulation time (TIME)

TIN - parameter used in temperature gradient

TIN0 - initial problem temperature (INIT)

TIN1 - parameter used in temperature gradient

TMCH - machine tolerance (ITER)

TOUTFL - parameter used in calculation of flow output

UPWGT - upwind weighting parameter

UPWGTA - upwind weighting factor for tracer solution (TRAC)

UPWGTB - upwind weighting factor for tracer solution

VLMAX - maximum liquid phase velocity

VTOT - total volume in problem

VVMAX - maximum vapor phase velocity

common/FAAC/

SSSOL - indicates if initial steady state solution is needed

VEQNO - contains version number of FEHMN code used

common/FAAC1/

IDATE - contains the date $(\mathrm{mm} / \mathrm{dd} / \mathrm{yr})$

\section{INCLUDE file COMBI}

common/FBB/

NELM(NELMD) - initially information about nodes in each element, later nodal connectivity information

NOP(NNOP) - matrix sparsity structure for LU decomposition

NAR(N0)

- array containing gauss elimination order for each node

ISTRW(N0)

- starting positions in $\mathrm{sx}(\mathrm{nr}, 9)$ array of finite element coefficients for each node

KA(N0) - contains boundary type information for each node

NELMDG(N0) - contains position of $(\mathrm{i}, \mathrm{i})$ element in connectivity array 
SX1(N0) - contains volume associated with each node

SX(NR,9) - contains finite element geometric coefficients necessary for heat and mass transfer simulation

SXS(NQ,6) - contains more finite element geometric coefficients (ie., those necessary for the stress module)

\section{common/FBS/}

$\operatorname{CORD}(\mathrm{N} 0,3)$

$\mathrm{XD}(8), \mathrm{YD}(8), \mathrm{ZD}(8)$ $\operatorname{SI}(8), \operatorname{ETA}(8), \operatorname{EXCI}(8)$

$\mathrm{XT}(8), \mathrm{YT}(8), \mathrm{ZT}(8)$

$\mathrm{W}(8,8)$

$\mathrm{WX}(8,8)$

WY $(8,8)$

$\mathrm{WZ}(8,8)$

$\mathrm{DR}(8)$

$\mathrm{DP}(6)$

WR $(8,8)$

$\mathrm{WXR}(8,8)$

WYR $(8,8)$

WZR $(8,8)$

$\mathrm{WP}(8,8)$

WXP $(8,8)$

WYP $(8,8)$

$\mathrm{WZP}(8,8)$
- contains the coordinates of each node

- global coordinates of the nodes in a finite element

- local coordinates in a finite element of the numerical integration points

- parameters needed in element calculations

- finite element shape functions

- derivative of shape functions with respect to $x$

- derivative of shape functions with respect to $y$

- derivative of shape functions with respect to $z$

- contains weights for integration points (bricks, rectangles)

- contains weights for integration points (prisms, triangles)

- finite element shape functions (rectangles)

- derivative of shape functions with respect to $x$ (rectangles)

- derivative of shape functions with respect to $y$ (rectangles)

- derivative of shape functions with respect to $z$ (rectangles)

- finite element shape functions (prisms)

- derivative of shape functions with respect to $x$ (prisms)

- derivative of shape functions with respect to $y$ (prisms)

- derivative of shape functions with respect to $z$ (prisms)

\section{INCLUDE File COMCI}

common/FCC/

DANL(N7) - derivative of liquid phase concentration with respect to total concentration

DANV(N7) - derivative of vapor phase concentration with respect to total concentration

$\mathrm{AKC}(\mathrm{N} 7)$ - tracer accumulation term derivative with respect to total concentration

$\mathrm{DRC}(\mathrm{N} 7)$ - tracer source term derivative with respect to total concentration

$\operatorname{DEEF}(\mathrm{N} 0)$ - derivative of energy accumulation with respect to energy variable

$\operatorname{DEPF}(\mathrm{NO})$ - derivative of energy accumulation term with respect to pressure 
$\mathrm{DMPF}(\mathrm{N} 0)$ - derivative of mass accumulation term with respect to pressure

$\operatorname{DMEF}(\mathrm{N} 0)$ - derivative of mass accumulation term with respect to energy variable

$\mathrm{DQ}(\mathrm{N} 0)$ - derivative of mass source term with respect to pressure

$\mathrm{DQH}(\mathrm{NO})$ - derivative of energy source term with respect to pressure

$\mathrm{DEQH}(\mathrm{N0})$ - derivative of energy source term with respect to energy variable

$\mathrm{DTPAE}(\mathrm{N} 0)$ - derivative of temperature with respect to energy variable

$\mathrm{DTPA}(\mathrm{N} 0)$ - derivative of temperature with respect to pressure

$\mathrm{DEVEF}(\mathrm{NO})$ - derivative of enthalpy with respect to energy variable

$\operatorname{DEVF}(\mathrm{NO})$ - derivative of enthalpy with respect to pressure

DRVPF(NO) - derivative of vapor relative permeability with respect to pressure

DRVEF(N0) - derivative of vapor relative permeability with respect to energy variable

DGLE(N0) - derivative of liquid mass gravity term with respect to energy variable

DGLP(N0) - derivative of liquid mass gravity term with respect to pressure

DELEF(N0) - derivative of liquid enthalpy with respect to energy variable

$\operatorname{DELF}(\mathrm{NO})$ - derivative of liquid enthalpy with respect to pressure

DRLPF(N0) - derivative of liquid relative permeability with respect to pressure

DRLEF(NO) - derivative of liquid relative permeability with respect to energy variable

DILE(N0) - derivative of liquid transmissibility with respect to energy variable

DILP(N0) - derivative of liquid transmissibility with respect to pressure

$\operatorname{ENLF}(\mathrm{NO})$ - liquid enthalpy

ENVF(N0) - vapor enthalpy

$\operatorname{DSTM}(\mathrm{N} 0)$ - steam mass

DENI(N0) - mass accumulation term

DENEI(N0) - energy accumulation term

DIL(NO) - liquid transmissibility

DIV(N0) - vapor transmissibility

ROLF(N0) - liquid density

ROVF(NO) - vapor density

$R L F(N 0)$ - liquid phase relative permeability

RVF(N0) - vapor phase relative permeability

$\operatorname{DENCI}(\mathrm{N} 7)$ - tracer accumulation term

GL(N0) - liquid phase gravity term

GV(No) - vapor phase gravity term

$\mathrm{DIVE}(\mathrm{N} 0)$ - derivative of vapor transmissibility with respect to energy variable

DIVP(N0) - derivative of vapor transmissibility with respect to pressure

DGVP(N0) - derivative of vapor mass gravity term with respect to pressure

DGVE(N0) - derivative of vapor mass gravity term with respect to energy variable

$\mathrm{DPCEF}(\mathrm{N} 0)$ - derivative of capillary pressure with respect to the energy variable

\section{INCLUDE file COMDI}

common/FDD/

THX(N0) - thermal conductivity $x$-direction 
THY(NO) - thermal conductivity y-direction

THZ(NO) - thermal conductivity z-direction

VOLUME(N0) - volume associated at each node

SK(NO)

- source strength of each node

$\operatorname{ESK}(\mathrm{N} 0)$

- inlet enthalpy associated with a source

QFLUX(N0) - heat flux at each node

QFLXM(N0) - heat flux impedance at each node

PNX(N3)

PNY(N3)

- permeability in the $\mathrm{x}$-direction, liquid velocity in the $\mathrm{x}$-direction, vapor velocity in the $x$-direction

- permeability in the $y$-direction, liquid velocity in the $y$-direction, vapor velocity in the $y$-direction

PNZ(N3) - permeability in the z-direction, liquid velocity in the $\mathrm{z}$-direction, vapor velocity in the $\mathrm{z}$-direction

PS(N0) - porosity at each node

EFLOW(N0) - energy flow at each source node

PHI(NO) - pressure at each node

PHO(N0) - last time step pressure at each node

$\mathrm{T}(\mathrm{N} 0)$

TO(N0)

$\mathrm{VF}(\mathrm{N} 0)$

- temperature at each node

- last time step pressure at each node

PFLOW(N0) - flowing pressure at each source node

DENEH(NO) - last time step energy accumulation term at each node

$\mathrm{QH}(\mathrm{NO})$

$\mathrm{S}(\mathrm{N} 0)$

$\mathrm{SO}(\mathrm{N} 0)$

- energy source term at each node

- liquid saturation at each node

WELLIM(NO) - well impedance at each source node

DENH(NO)

$\operatorname{DENJ}(\mathrm{NO})$

- last time step mass accumulation term at each node

DENEJ(NO)

DENR(N0)

- last time step mass accumulation time derivative at each node

CPR(NO)

- last time step energy accumulation time derivative at each node

$\mathrm{PCP}(\mathrm{N} 0)$

- rock density at each node

- rock specific heat at each node

- capillary pressure at each node

\section{common/FDDI/}

NSKW(NO) - contains nodes for print-out

\section{common/FDD1/}

AN(N7) - total tracer concentration at each node

ANL(N7) - liquid tracer concentration at each node

ANV(N7) - vapor tracer concentration at each node

CNSK(N7) - tracer concentration source term at each node 
T1SK(N7) - time when the tracer source term is activated at each node

T2SK(N7) - time when the tracer source term is terminated at each node

$\mathrm{RC}(\mathrm{N} 7)$ - tracer source term at each node

$\mathrm{DENCH}(\mathrm{N} 7)$ - last time step tracer accumulation term at each node

$\operatorname{DENCJ}(\mathrm{N} 7)$ - last time step tracer accumulation derivative term at each node

ANLO(N7) - last time step total tracer concentration at each node

$\mathrm{FC}(\mathrm{N} 7) \quad$ - tracer equation residual at each node

$\mathrm{TCX}(\mathrm{N} 7) \quad$ - tracer diffusion term in the $\mathrm{x}$-direction

$\mathrm{TCY}(\mathrm{N} 7) \quad$ - tracer diffusion term in the $y$-direction

TCZ(N7) - tracer diffusion term in the z-direction

$\mathrm{CM}(10) \quad$ - total tracer mass for each specie

CM0(10) - initial total tracer mass for each specie

QCIN (10) - total injected tracer mass for each specie

QOUT(10) - total produced tracer mass for each specie

A1ADF(10) - alpha1 parameter for each specie(nonlinear adsorbtion)

A2ADF(10) - alpha2 parameter for each specie (nonlinear adsorbtion)

$\operatorname{BETADF}(10)$ - beta parameter for each specie (nonlinear adsorbtion)

A1R

- parameterin reaction rate model

A2R

- parameterin reaction rate model

A3R

- parameterin reaction rate model

A4R

- parameterin reaction rate model

$\mathrm{RP} 1 \mathrm{~F}(25)$

- parameter in relative permeability model

$\mathrm{RP} 2 \mathrm{~F}(25)$

$\mathrm{RP} 3 \mathrm{~F}(25)$

- parameter in relative permeability model

$\mathrm{RP} 4 \mathrm{~F}(25)$

- parameter in relative permeability model

$\mathrm{RP} 5 \mathrm{~F}(25)$

$\mathrm{RP} 6 \mathrm{~F}(25)$

- parameter in relative permeability model

$\mathrm{RP} 7 \mathrm{~F}(25)$

- parameter in relative permeability model

$\mathrm{RP} 8 \mathrm{~F}(25)$

- parameter in relative permeability model

$\mathrm{RP9F}(25)$

- parameter in relative permeability model

$\mathrm{RP} 10 \mathrm{~F}(25)$

- parameter in relative permeability model

$\mathrm{RP} 11 \mathrm{~F}(25)$

- parameter in relative permeability model

RP12F(25)

- parameter in relative permeability model

RP13F(25)

- parameter in relative permeability model

$\mathrm{RP} 14 \mathrm{~F}(25)$

- parameter in relative permeability model

RP15F(25)

- parameter in relative permeability model

$\mathrm{RP} 16 \mathrm{~F}(25)$

- parameter in relative permeability model

$\mathrm{RP} 17 \mathrm{~F}(25)$

$\mathrm{CP} 1 \mathrm{~F}(10)$

CP2F(10)

- parameter in relative permeability model

CP3F(10)

$\operatorname{DIT}(300)$

- parameter in relative permeability model

- parameter in relative permeability model

- parameter in capillary pressure model

- parameter in capillary pressure model

- parameter in capillary pressure model

- array containing time step changes 
NSPECI - number of species for tracer solution

NSP - current specie number

NPN - parameter used in storing tracer data

NPT(10) - storage parameter used in tracer solution

IADD $(10) \quad$ - iteration count for tracer solution

IADDT(10) - iteration count used for linear equation solver in tracer solution

IADSF(10) - adsorption model used in tracer solution

ICAPT(10) - capillary pressure model

IRLPT(10) - relative permeability model

ICNS(10) - liquid or gas phase tracer model

ICRATE - reaction rate model

ITC(100) - array containing information used in time step changes

\section{common/FDD2/}

PHINI(N8) - initial pressure at each node

PSINI(N8) - initial porosity at each node

DPORP(N8) - derivative of porosity with respect to pressure at each node

DPORT(N8) - derivative of porosity with respect to temperature at each node

TINI(N8) - initial temperature at each node

SIGINI - parameter used in rock deformation model

PHYDRO - parameter used in rock deformation model

THEXP - parameter used in rock deformation model

YOUNG - parameter used in rock deformation model

AMGANG(N8) - parameter used in gangi model at each node

PGANGI(N8) - parameter used in gangi model at each node

\section{common/FICE/}

SII(N6) - ice saturation at each node

SIO(N6) - last time step value of ice saturation

TMELT - freezing temperature of water 
ICES(N6) - state of ice at each node

IIEOS(N0) - thermodynamics set at each node

IEOS(N0) - phase state of fluid at each node

IEOSC - phase change parameter

IPORF(N0) - deformation model at each node

IRLP(N0) - relative permeability model at each node

ICAP(N0) - capillary pressure model at each node

\section{INCLUDE fle COMEI}

common/FEE/

A(LDN) - array containing the Jacobian matrix

common/FFF/

$\mathrm{B}(\mathrm{NBD}) \quad$ - array containing the incomplete LU decomposition of the Jacobian matrix

\section{common/FHH/}

IRB(N0) - array containing the reordered node numbers

$\operatorname{IIRB}(\mathrm{N0}) \quad$ - inverse of irb

NOPT(N0) - array indicating active variables

NPVT(N0) - pivot information for the LU decomposition matrix

IG(N0) - variable order LU decomposition information

$\operatorname{PIV}(\mathrm{N} 0,9)$ - the pivot elements of the LU decomposition

\section{INCLUDE file COMFI}

DMC(N4) - derivative of mass accumulation term with respect to gas at each node DEC(N4) - derivative of energy accumulation term with respect to gas at each node DCP(N4) - derivative of gas accumulation term with respect to pressure at each node $\mathrm{DCE}(\mathrm{N} 4)$ - derivative of gas accumulation term with respect to energy at each node $\mathrm{DQC}(\mathrm{N} 4)$ - derivative of mass source term with respect to gas DEQC(N4) - derivative of energy source term with respect to gas DCQC(N4) - derivative of gas source term with respect to gas 

DENPCI(N4) - gas accumulation term at each node
DENPCH(N4) - last time step value of the mass accumulation term at each node
DIVC(N4)
- derivative of vapor transmissibility with respect to gas
DGVC(N4)
- derivative of vapor gravity term with respect to gas
$\operatorname{DCVCF}(\mathrm{N} 4)$
- derivative of gas concentration with respect to gas
$\operatorname{DCVEF}(\mathrm{N} 4)$
- derivative of gas concentration with respect to energy
DCVF(N4)
- derivative of gas concentration with respect to pressure
$\operatorname{DEVCF}(\mathrm{N} 4)$
CNVF(N4)
- derivative of energy with respect to gas
DILC(N4)
- concentration of gas in the vapor phase
DGLC(N4)
$\operatorname{ESKC}(\mathrm{N} 4)$
$\mathrm{DCLCF}(\mathrm{N} 4)$
- derivative of liquid transmissibility with respect to gas
$\operatorname{DCLEF}(\mathrm{N} 4)$
$\operatorname{DCLF}(\mathrm{N} 4)$
$\operatorname{DELCF}(\mathrm{N} 4)$
CNLF(N4)
$\mathrm{QC}(\mathrm{N} 4)$
$\mathrm{DCC}(\mathrm{N} 4)$
DTPAC(N4)
- derivative of liquid gravity term with respect to gas
- source term for gas equation
- derivative of liquid concentration with respect to gas
- derivative of liquid concentration with respect to energy
- derivative of liquid concentration with respect to pressure
- derivative of liquid energy with respect to gas
- gas concentration in the liquid phase
- source term for the gas equation
- derivative of gas accumulation term with respect to gas
DENPCJ(N4) - last time step accumulation term of gas equation
$\mathrm{DCQH}(\mathrm{N} 4)$
$\mathrm{PCI}(\mathrm{N} 4)$
- derivative of gas source term with respect to energy
$\mathrm{PCIO}(\mathrm{N} 4)$
DIFC
- gas pressure
- last time step gas pressure
- noncondensible gas mass balance error

\section{INCLUDE file COMGI}

\section{common/FGG/}

$\operatorname{DFMP}(\mathrm{NN})$ - derivative of mass accumulation term with respect to pressure for neighbor nodes

$\mathrm{DFME}(\mathrm{NN})$ - derivative of mass accumulation term with respect to energy for neighbor nodes

DFEP(NN) - derivative of energy accumulation term with respect to pressure for neighbor nodes

$\operatorname{DFEE}(\mathrm{NN}) \quad$ - derivative of energy accumulation term with respect to energy for neighbor nodes

DFMPC(NN) - derivative of mass accumulation term with respect to gas for neighbor nodes

$\mathrm{DFEPC}(\mathrm{NN})$ - an rivative of energy accumulation term with respect to gas for neighbor nodes

DFPCP $(\mathrm{NN})$ - derivative of gas accumulation term with respect to pressure for neighbor nodes

DFPCE(NN) - derivative of gas accumulation term with respect to energy for 
neighbor nodes

DFPCPC(NN) - derivative of gas accumulation term with respect to gas for neighbor nodes

BP(N3) - array of Newton-Raphson residuals, after solution, an array of Newton-Raphson corrections

BPC(N3) - array of Newton-Raplison residuals for gas, after solution, an array of Newton-Raphson corrections

\section{INCLUDE fle COMHI}

\section{common/DUALP/}

VOLF1(N5) - volume fraction at each node for the first matrix layer VOLF2(N5) - volume fraction at each node for the second matrix layer APUV1(N5) - area per unit volume for the first matrix layer WB11(N5) - array needed to store intermediate dual porosity results WB12(N5) - array needed to store intermediate dual porosity results WB21(N5) - array needed to store intermediate dual porosity results WB22(N5) - array needed to store intermediate dual porosity results TB11(N5) - array needed to store intermediate dual porosity results TB12(N5) - array needed to store intermediate dual porosity results TB21(N5) - array needed to store intermediate dual porosity results TB22(N5) - array needed to store intermediate dual porosity results A21MPF(N5) - array needed to store intermediate dual porosity results A21MEF(N5) - array needed to store intermediate dual porosity results A21EPF(N5) - array needed to store intermediate dual porosity results A21EEF(N5) - array needed to store intermediate dual porosity results A32MPF(N5) - array needed to store intermediate dual porosity results A32MEF(N5) - array needed to store intermediate dual porosity results A32EPF(N5) - array needed to store intermediate dual porosity results A32EEF(N5) - array needed to store intermediate dual porosity results RB2MF(N5) - array needed to store intermediate dual porosity results RB2EF(N5) - array needed to store intermediate dual porosity results RB3MF(N5) - array needed to store intermediate dual porosity results RB3EF(N5) - array needed to store intermediate dual porosity results

\section{INCLUDE file COMII}

\section{common/COEFF/}

CEL $(20,2)$ - polynomial coefficients for liquid water enthalpy equations $\mathrm{CRL}(20,2)$ - polynomial coefficients for liquid water density equations $\operatorname{CEV}(20,2)$ - polynomial coefficients for vapor water enthalpy equations 
$\mathrm{CRV}(20,2)$ - polynomial coefficients for vapor water density equations

CVL $(20,2)$ - polynomial coefficients for liquid water viscosity equations

CVV $(20,2)$ - polynomial coefficients for vapor water viscosity equations

TSA0 - polynomial coefficient for saturation temperature equation

TSPA1 - polynomial coefficient for saturation temperature equation

TSPA2 - polynomial coefficient for saturation temperature equation

TSPA3 - polynomial coefficient for saturation temperature equation

TSPA4 - polynomial coefficient for saturation temperature equation

TSB0 - polynomial coefficient for saturation temperature equation

TSPB1 - polynomial coefficient for saturation temperature equation

TSPB2 - polynomial coefficient for saturation temperature equation

TSPB3 - polynomial coefficient for saturation temperature equation

TSPB4 - polynomial coefficient for saturation temperature equation

PSA0 - polynomial coefficient for saturation pressure equation

PSTA1 - polynomial coefficient for saturation pressure equation

PSTA2 - polynomial coefficient for saturation pressure equation

PSTA3 - polynomial coefficient for saturation pressure equation

PSTA4 - polynomial coefficient for saturation pressure equation

PSB0

PSTB1

PSTB2

PSTB3

PSTB4

- polynomial coefficient for saturation pressure equation

- polynomial coefficient for saturation pressure equation - polynomial coefficient for saturation pressure equation - polynomial coefficient for saturation pressure equation - polynomial coefficient for saturation pressure equation

common/COEFF1/

PMAX(3) - maximum pressure allowed for each coefficient set $\operatorname{PMIN}(3)$ - minimum pressure allowed for each coefficient set

$\operatorname{TMAX}(3)$ - maximum temperature allowed for each coefficient set TMIN(3) - minimum temperature allowed for each coefficient set

\section{common/COEFF2/}

EW1 - coefficient used in simplifying thermodynamics relations

EW2 - coefficient used in simplifying thermodynamics relations

EW3 - coefficient used in simplifying thermodynamics relations

EW4 - coefficient used in simplifying thermodynamics relations

EW5 - coefficient used in simplifying thermodynamics relations

EW6 - coefficient used in simplifying thermodynamics relations

EW7 - coefficient used in simplifying thermodynamics relations

EW8 - coefficient used in simplifying thermodynamics relations

EW9 - coefficient used in simplifying thermodynamics relations

EW10 - coefficient used in simplifying thermodynamics relations

EW11 - coefficient used in simplifying thermodynamics relations 
EV1 - coefficient used in simplifying thermodynamics relations

EV2 - coefficient used in simplifying thermodynamics relations

EV3 - coefficient used in simplifying thermodynamics relations

EV4 - coefficient used in simplifying thermodynamics relations

EV5 - coefficient used in simplifying thermodynamics relations

EV6 - coefficient used in simplifying thermodynamics relations

EV7 - coefficient used in simplifying thermodynamics relations

EV8 - coefficient used in simplifying thermodynamics relations

EV9 - coefficient used in simplifying thermodynamics relations

EV10 - coefficient used in simplifying thermodynamics relations

EV11 - coefficient used in simplifying thermodynamics relations

\section{INCLUDE file DAVIDI}

\section{common/DAVID1/}

IRDOF - reduced degree of freedom model used

ISLORD - parameter used in the reduced degree of freedom model

IBACK $\quad-$ LU factorization save parameter

ICOUPL - number of SOR iterations

ITEST - parameter used in the reduced degree of freedom model

\section{common/DAVID2/}

NMAT(9) - array used in the reduced degree of freedom method NRHS(3) - array used in the reduced degree of freedom method NMAT32(4) - array used in the reduced degree of freedom method

\section{Subroutine Structure for FEHMN}

Figure 4 presents the general subroutine call tree structure for the code FEHMN. Due to its complexity, the entire structure could not fit within a page, hence it is broken into several branches. As an example of the tree's use we trace the call structure from GZ (the main program), which calls INPUT (0003), which calls CONCEN(0021), which calls CSOLVE (0026), which calls CNSWER (0028) which calls THERMC (0028). At subroutine THERMC, the tree is directed to line 0024 where the calls from THERMC have been previously explained. We see that THERMC calls DABS. This was only one path of many possible; all paths may be traversed in a way analogous to above. 


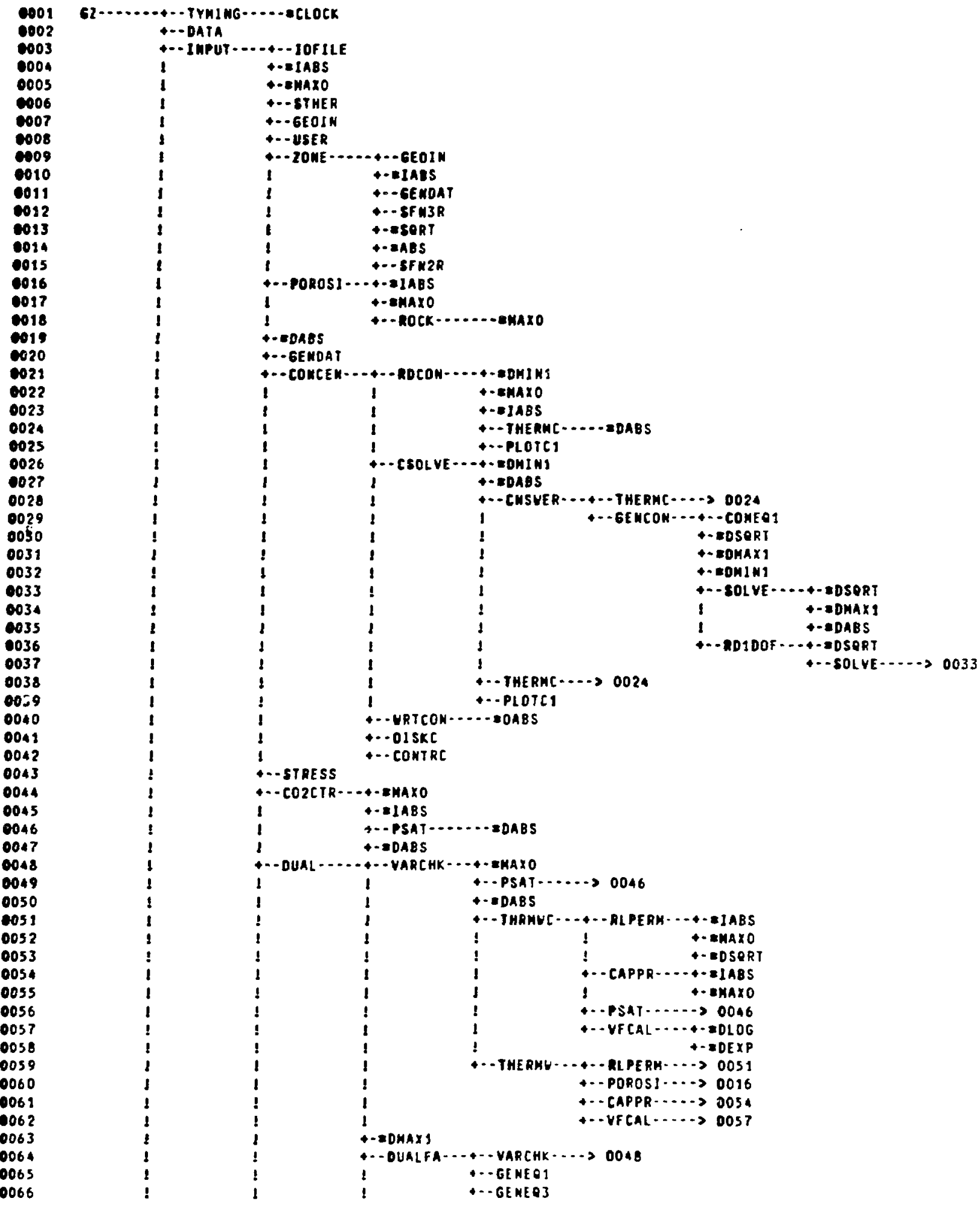

Fig. 4. Structure for subroutine calls for FEHMN. 


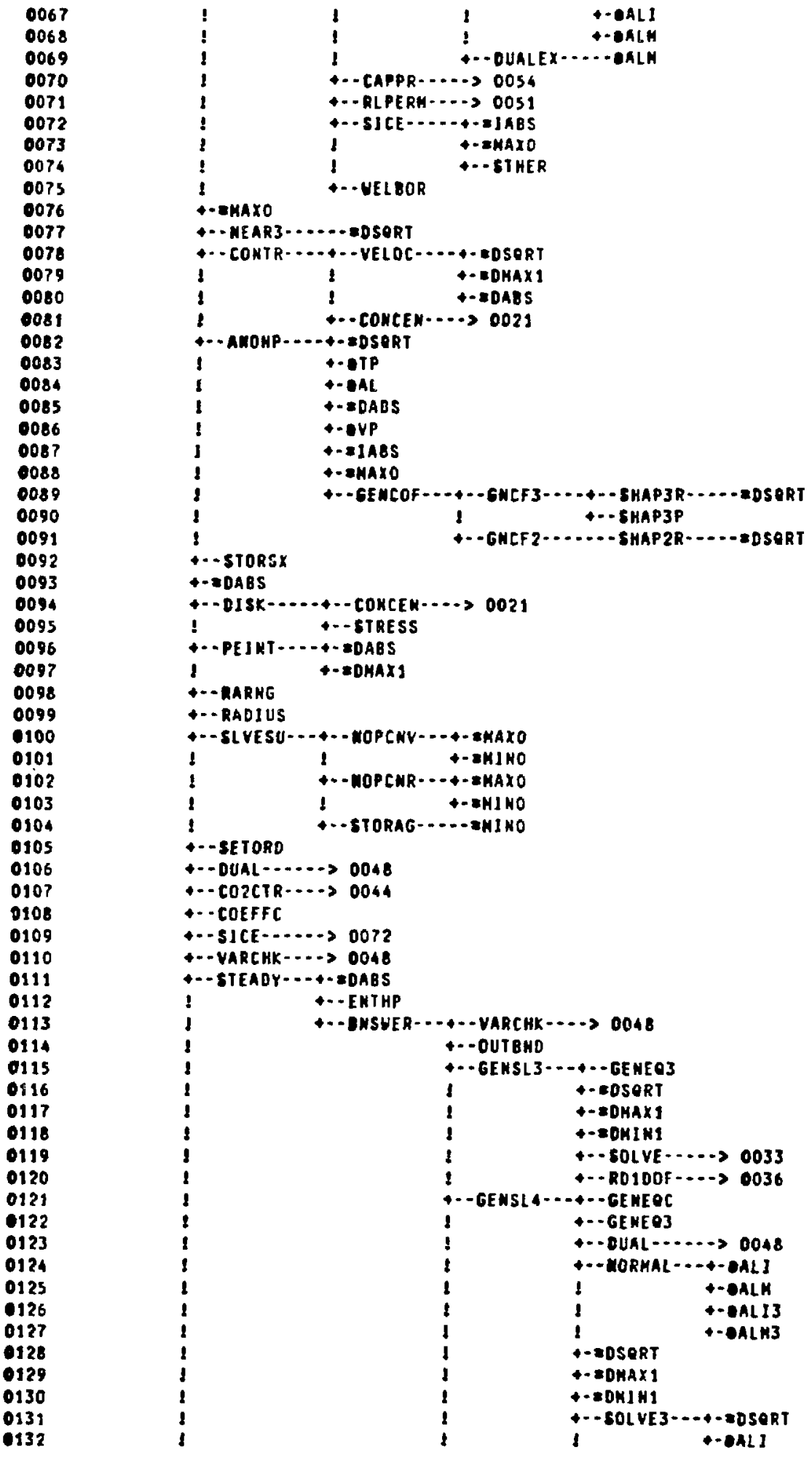

Fig. 4. (Continued) 


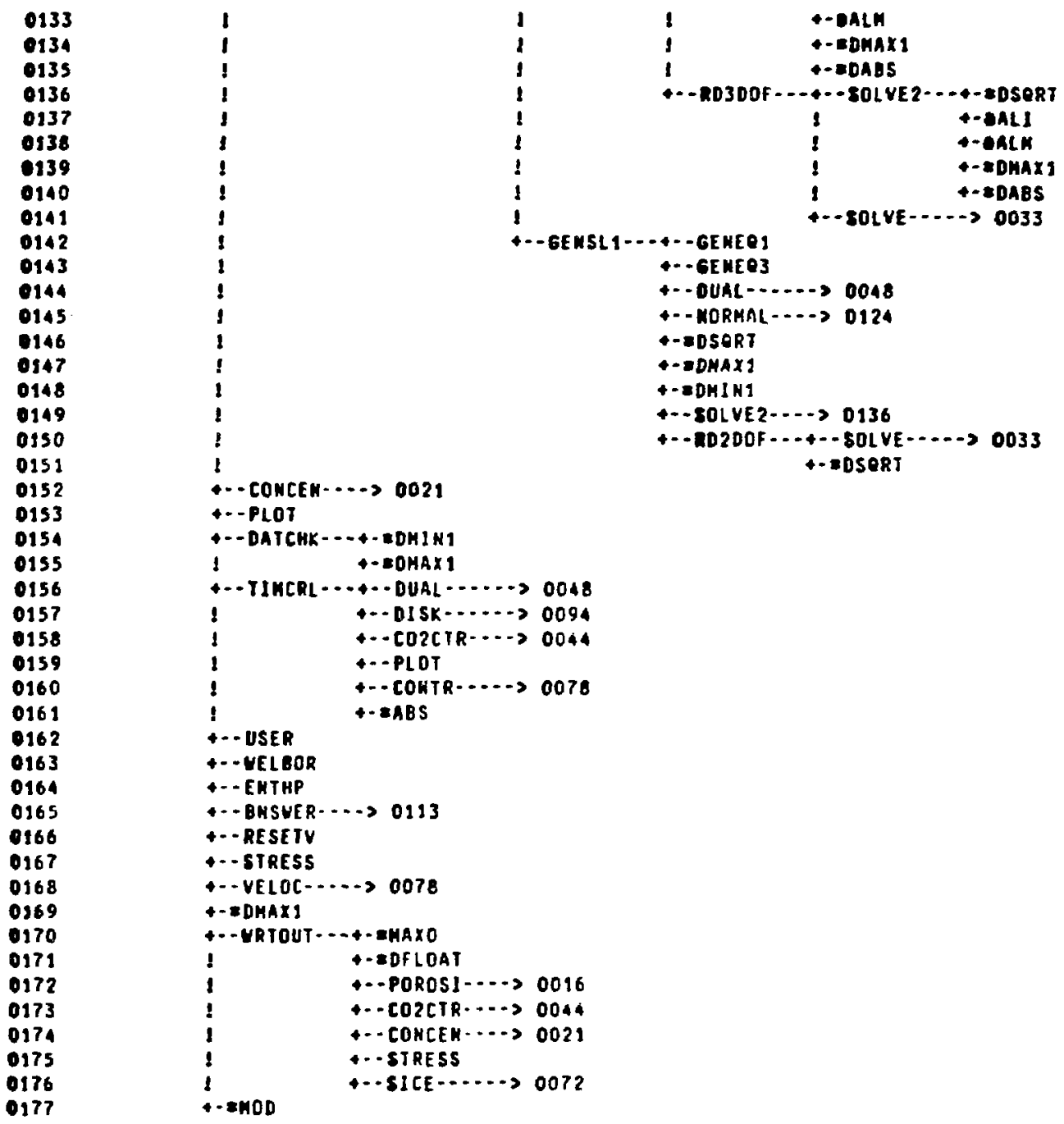

Fig. 4. (Continued) 


\section{USER'S MANUAL}

This section provides the instructions necessary for using the code FEHMN. Section A describes an automatic mesh generation code, GENMSH, that can be used to generate the finite element mesh input required by FEHMN. Section B describes in detail the input macro-commands for FEHMN. A graphics postprocessor is described in Section $\mathrm{C}$ and several example problems are given in Section D.

\section{A. A utomatic Mesh Generation}

The code GENMSH, which is designed to be used in conjunction with the finite element code FEHMN, operates by dividing a user-defined solution space into a prescribed number of finite elements. The solution space may be either two or three dimensicial. In order to simplify geometric considerations, the solution space is broken up into a rumber of "blocks." Each block is then further divided into "elements." A given block uses the same "type" of element throughout, although the elements need not be of the same size. Different blocks may use different types of elements. The choice of elements available in two dimensions for output to FEHMN are 4-noded quadrilaterals and 3-noded triangles. In three dimensions, the choice is 8-noded quadrilateral polyhedrons or 3-noded triangular prisms, shown schematically in Fig. 5.

The solution space, and each block, is defined by giving the corner and/or midpoint nodes. This amounts to specifying 4 or 8 nodes per block in 2-D problems and 8 or 20 nodes per block in 3-D problems as shown in Fig. 6. The same block definition is needed for both quadrilateral and triangular elements. After discretizing the solution space and the blocks, it is necessary to specify the desired division of each block into elements. This is done by specifying the :elative weights of each subdivision in each direction for each block. The reader is reierred to An Introduction to Finite Element Computations by Hinton and Owen (pp. 328-346) for the details of the procedure and the algorithm. Note that material properties are specified at each node in FEHMN, unlike conventional finite element procedure.

A detailed description of the input to the code follows. The input must be placed in a file called MDAT. Examples of the MDAT file are given in Section D. The output is found on a file called GEOM.DAT. The output consists of a list of nodal coordinates and elements in a suitable format for the code FEHMN. Free format is used for the input. In addition, a zone definition of the input blocks is provided in file ZONE. The files GEOM.DAT and 


$$
\text { 2-D }
$$
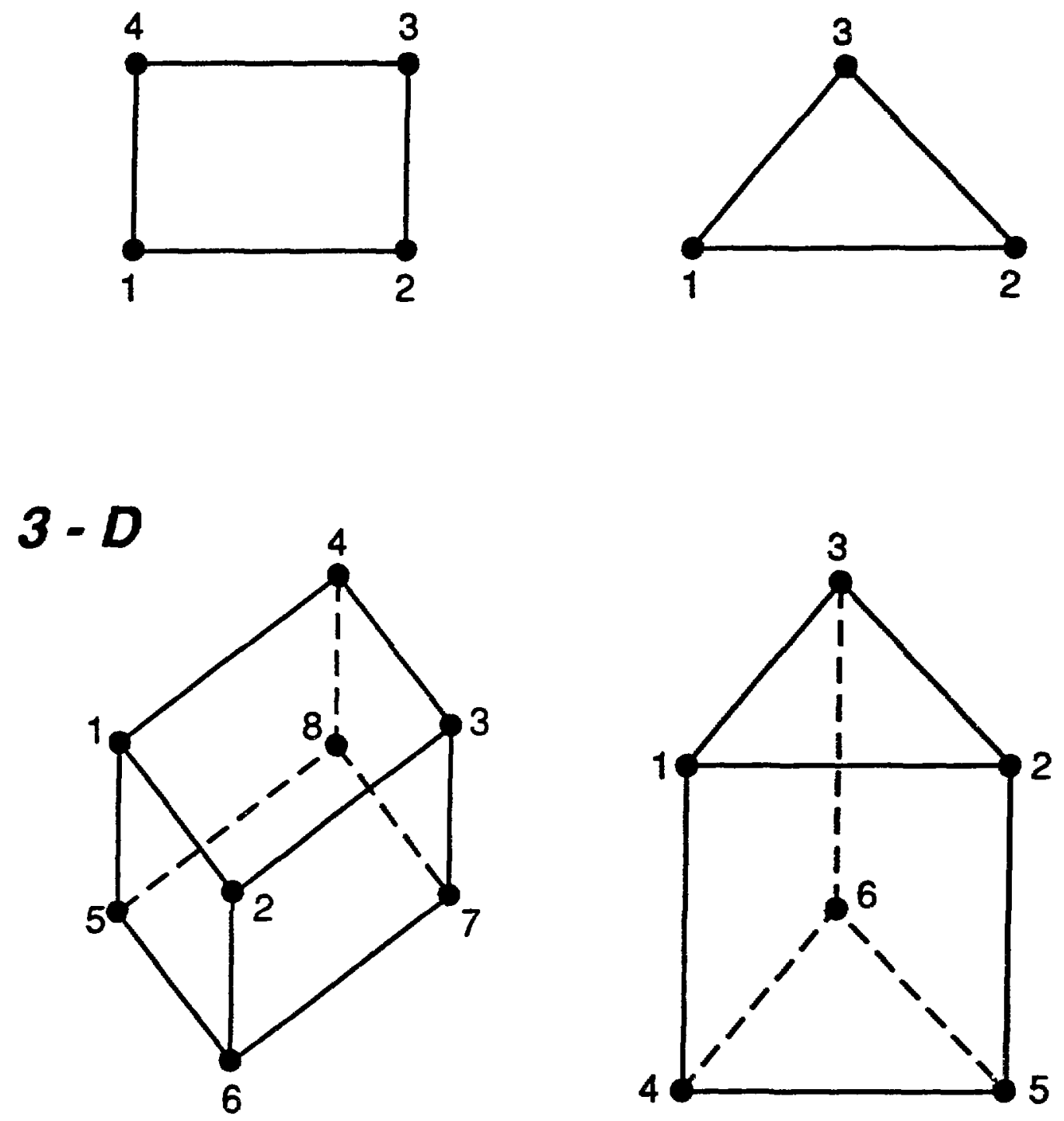

Fig. 5. Elements available with FEHMN in 2-D and 3-D problems showing numbering convention. 
2- D

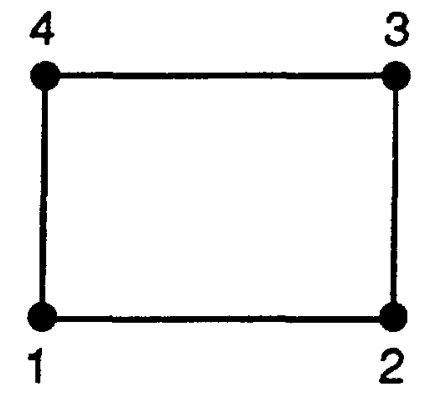

4 - node

3-D

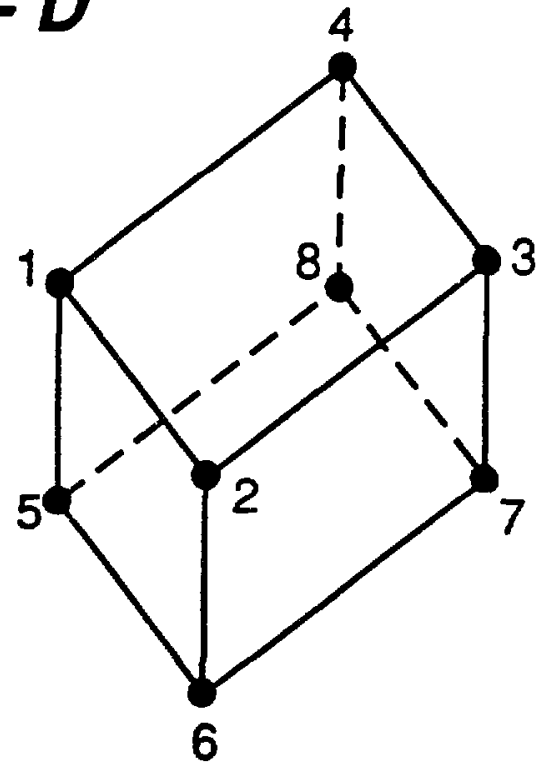

8 - node

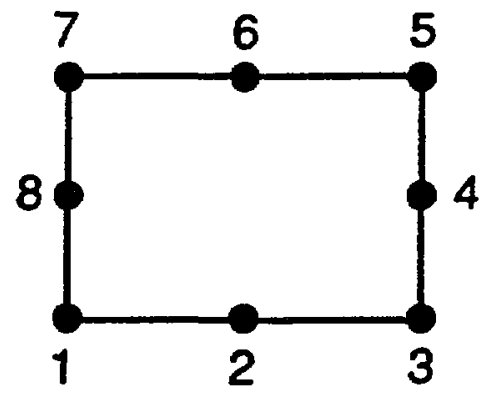

8 - node

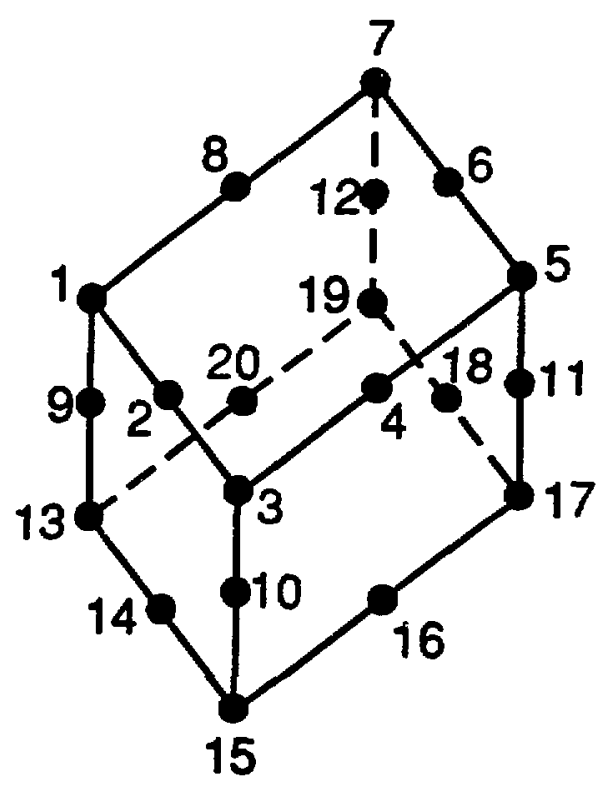

20 - node

Fig. 6. Nodal ordering for defining 2-D and 3-D domain blocks for input to GENMSH. 
ZONE may be used directly in the input file for FEHMN as they already include the appropriate macro command headings.

Group 1

Title, up to 80 characters long

Group 2

NPOIN, NELEM, LNODE, IT, NDIME, KF, NRN

NPOIN - total number of points used to define the solution space

NELEM - number of blocks used to define the solution domain

LNODE - number of nodes per block used to define the solution domain

IT - element triangulation parameter, if IT $=0$, then no element splitting occurs, if $\mathrm{IT} \neq 0$, then triangle elements are generated in 2-D and prism elements are generated in 3-D.

NDIMB - dimension of problem (2 or 3 )

$\mathrm{KF} \quad$ - determines the vertical axis direction in 2-D. If $K F<0$ then the $y$-axis is positive downward. If $K F \geq 0$, the $y$-axis is positive upward in 3-D, the $z$-axis is always positive upward. See note on plotting below.

NRN - renumber parameter. If NRN $\neq 0$ the code will renumber to minimize the band width. If $\mathrm{NRN}=0$ then no renumbering will occur.

Group 3 (NUMEL, Block Times)

NUMEL, (LNODS(NUMEL,INOD), INODE = 1, LNODE)

NUMEL - Block number

LNODS (NUMEL, INOD) - LNODE nodes defining the block NUMEL

Group 4

CORDX (I), I = 1, NPOIN

CORDX (I) - $\mathrm{x}$-coordinates of points used to define the solution space

Group 5

CORDY (I), I = 1 , NPOIN

CORDY (I) - $y$-coordinates of points used to define the solution space

Group 6 (only if NDIME $=3$ )

CORDZ (I). I = 1 , NPOIN

CORDZ (I) - z-coordinates of points used to define the solution space NOTE: Groups 7-10 are repeated for each block. 


\section{Group 7}

KBLOC, NDIVX, NDIVY, NDIVZ

KBLOC - block number

NDIVX - number of divisions in $x$-direction

NDIVY - number of divisions in y-direction

NDIVZ - number of divisions in z-direction (only if NDIME = 3).

NOTE: The number of nodes in a direction $=$ number of divisions +1 .

Group 8

WEITX (I), I = 1 , NDIVX

WEITX (I) - weighting of the Ith division in the $x$-direction in block KBLOC

Group 9

WEITY (I), I = 1, NDIVY

WEITY (I) - weighting of the Ith division in the $y$-direction in block KBLOC

Group 10

WEITZ (I), I = 1, NDIVZ (only if NDIME = 3)

WEITZ (I) - weighting of the Ith division in the $\mathrm{z}$-direction in block KBLOC

Group 11 (only if NDIME $=3$ )

$\mathrm{XV}, \mathrm{YV}, \mathrm{ZV}$

$\mathrm{XV}$ - $\mathrm{x}$-coordinate of viewing point for 3-D plot

YV - y-coordinate of viewing point for 3-D plot

ZV - z-coordinate of viewing point for 3-D plot

NOTE: $\mathrm{XV}=300, \mathrm{YV}=300, \mathrm{ZV}=50$ gives a good viewing arigle for many problems.

Group 12 (only if NDIME $=3$ )

XMIN, XMAX, YMIN, YMAX, ZMIN, ZMAX

XMIN - minimum $\mathrm{x}$-cordinate for plot of mesh

XMAX - maximum $x$-coordinate for plot of mesh

If XMIN = XMAX then the limits will be determined from the generated mesh

YMIN - minimum y-cordinate for plot of mesh

YMAX - maximum y-coordinate for plot of mesh

If YMIN = YMAX then the limits will be determined from the generated mesh

ZMIN - minimum z-cordinate for plot of mesh

ZMAX - maximum z-coordinate for plot of mesh

If $\mathrm{ZMIN}=\mathrm{ZMAX}$ then the limits will be determined from the generated mesh 
Group 13 (optional)

NREF, XR, YR, ZR, IRFN

NREF - reference node for refinement

XR - $\mathrm{x}$-coordinate of reference node

$Y R \quad$ - $y$-coordinate of reference node

ZR - z-coordinate of reference node

IRFN - refinement level for reference node

If NREF $<0$ then the node closest to the reference coordinates is used as the reference node. If IRFN $<0$ then the node closest to the reference coordinates is used as the reference node and the coordinates set to the reference coordinates.

The refinement level is ABS (IRFN). If the refinement level is 1 then the elements surrounding the reference node are subdivided once and new elements created. A refinement level of 2 means this is done twice, and so on.

Note on plotting. If $K F \neq 0$ in Group 2 a plot of the mesh is provided, and if $A B S(K F)>1$ node numbers are printed on the plot.

\section{B. Macro Command Input For FEHMN}

The finite element heat and mass transfer code (FEHMN) contains a macro control structure for data input that offers added flexibility to the input process. Blocks of data can be entered in any order, and any blocks unnecessary to a particular problem can be disregarded. All entries are free format, which adds flexibility, but requires that values be entered for all input variables (no assumed null values). As an aid to the user, the capabilities of FEHMN are summarized in Table IV with reference to the macro commands. Values of parameters are entered either by node number or a geometric description (macro command ZONE). The user is encouraged to read the macro ZONE description. 
I. Mass, energy balances in porous media
A. Variable rock properties (ROCK)
B. Variable permeability (PERM)
C. Variable thermal conductivity (COND)
D. Variable fracture properties, dual porosity (DUAL)

II. Multiple components available (NCON)
B. Up to 10 passive tracers available (TRAC)
A. Air water mixture available, fully coupled to heat and mass transfer
C. Several different capillary pressure models (CAP)
D. Several different relative permeability models (RLP)

III. Equation of state flexibility inherent in code (EOS)

IV. Psuedo-stress models available
A. Linear porosity deformation (PPOR)
B. Gangi stress model (PPOR)

V. Numerics
A. Finite element with multiple element capabilities (ELEM)
B. Short form input methods available (COOR, ELEM)
C. Flexible properties assignment (ZONE)
D. Flexible solution methods
1. Upwinding, implicit solution available (CTRL)
2. Iteration control adaptive strategy (ITER)

VI. Flexible time step and stability control (TIME)

TABLE V. FEHMN Files

$\begin{array}{ll}\text { TAPEn } 1(1 \leq \mathrm{n} 1 \leq 10) & \text { input file (user supplied) } \\ \text { TAPEn2 }(11 \leq \mathrm{n} 2 \leq 20) & \text { output file (code generated) } \\ \text { TAPEn3 }(21 \leq \mathrm{n} 3 \leq 30) & \text { initial value tape (read a restart, user supplied) } \\ \text { TAPEn4 }(31 \leq \mathrm{n} 4 \leq 40) & \begin{array}{l}\text { final value tape (write a restart, code generated) } \\ \text { time history of variables (code generated) } \\ \text { FE.HIS }\end{array} \\ \begin{array}{l}\text { contour plot data (code generated) } \\ \text { contains program information of the code crashes (code } \\ \text { FE.MIS }\end{array} & \begin{array}{l}\text { generated) } \\ \text { tracer time history data (code generated) } \\ \text { storage of finite element coefficients (user supplied or } \\ \text { code generated) } \\ \text { FE.TRC }\end{array} \\ \text { contains input analysis }\end{array}$


Although most input data are contained in the input deck, some information must first be entered from the terminal. Most of the information required before the input deck can be read pertains to input and output files and is prompted for from within the program. Figure 7 shows the output for a typical FEHMN run on a CRAY computer. All files are of the form TAPEn, where $n$ is the tape (unit) number. Table $V$ contains a description of FEHMN files. The terminal input follows:

(free format) INPT, IOUT, IREAD, ISAVE, IDC

INPT - unit number of the input file

IOUT - unit number of the file to which nodal information (pressures, enthalpies, etc.) is printed

IREAD - unit number of file on which initial values of pressure and enthalpy are found. (If IREAD $=0$, initial values are generated from input data. See macro control statement PRES)

ISAVE - unit number of file on which final time nodal information is printed for restart purposes (if ISAVE $=0$, no restart file is created).

IDC - user subroutine parameter

if IDC $=0$ the code makes no USER subroutine calls

if IDC $\neq 0$ the code calls subroutine USER (user defined) at each time step.

The form is CALL USER (IDC).

In Fig. 8, the user entered data is after the "?". Here INPT $=1$, IOUT $=11$, IREAD $=$ 0 , ISAVE $=0$, and IDC $=0$. The remainder of the data is read from unit INPT (which was defined above).

The macro command structure makes use of a set of control statements recognized by the input module of the program. When a macro control statement is encountered in the input deck, a certain set of data is expected and read from the input deck. In this way, the input is divided into separate, unordered blocks of data. The input deck is therefore a collection of macro control statements, each followed by its associated data block, as the example input files (Section D) show. Note that, although the input is free format, macro control statements must appear in the first four columns of a line. Table VI lists the macro control statements with brief descriptions of the data associated with each. The macro control statements may be in any order, although the data associated with eacli macro control statement must follow with the prescribed format. Some statements are necessary and others are optional, as indicated in Table VI.

A detailed list of the rnacro control statements and the input variables associated with each is given in Table VII. In Section D example input files are given. 


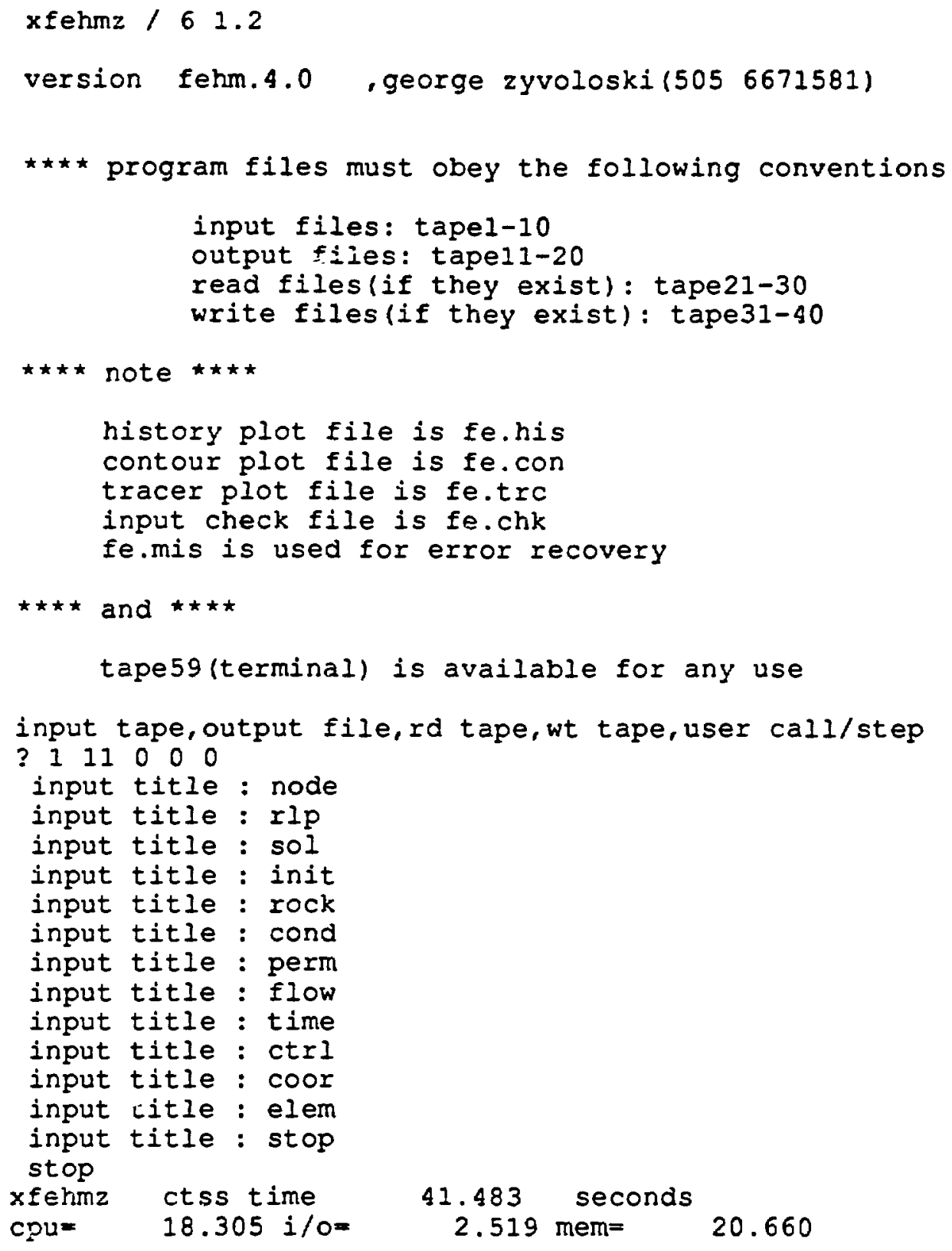

Fig. 7. 'Terminal output for a FEHMN run on a CRAY computer. 
CAP

COOR

COND

CONT

CTRL

DUAL

ELEM

EOS

FLOW

HFLX

INIT $^{\star}$

ITER

NGAS

NODE

PERM

PPOR

PRES

RLP

ROCK

SOL

STEA

STOP

TEXT

TIME

TRAC

ZONE capillary pressure data, optional.

node coordinate data, required.

thermal conductivity data, required.

contour plot data, optional.

program control parameters, required.

input for dual porosity solution, optional.

element node data, required.

equation of state data, optional.

flow data, optional.

heat flux data, optional.

initial value data, optional.

iteration parametes, optional.

noncondensible gas (air) data, optional.

node numbers for output and time histories, required.

permeability and velocity data, required.

pressure and temperature dependent porosity and permeability, optiona initial pressure and enthalpy data, optional.

relative permeability data, optional (required for 2-phase problem).

rock density, specific heat, and porosity data, required.

solution specifications, required.

steady state solution generated for initial variable field, optional.

signals the end of input, required.

text input, optional.

time step and time of simulation data, required.

tracer data, optional.

geometric definition of grid for input definition, optional.

*One or both INIT and PRES macros are necessary.

The user is encouraged to look at the file FE.CHK. This file contains information on where the maximum and minimum input parameters occur and suggestions on decreasing the storage requirements for the run; it also summarizes initial mass and energy values.

Many input parameters such as porosity or permeability vary throughout the grid and need to be assigned different values on different nodes. This is accomplished in two ways. The first is a nodal loop-type definition:

$$
\text { JA,JB,JC,PROP1,PROP2, ... }
$$

where 
Prop1,Prop2 - properties to be assigned to the nodes.

In the input blocks to follow one or more properties are manually entered in the above structure. When a value of 0 is entered for any JA, that input block is terminated and the code proceeds to the next group or control statement.

The nodal definition above is useful in simple geometries where the node numbers are easily found. The boundary nodes, in this case come at regular node intervals and the increment counter JC can be adjusted so the boundary conditions are easily entered.

In more complicated geometries, such as 3-D grids, the node numbers are often difficult to determine. Here a geometric description is preferred. To enable the geometric description the control statement ZONE (p. 74) is used in the input file before the other property macro statements occur. The input macro ZONE requires the specification of the coordinates of 4-node parallelograms for 2-D problems and the 8-node polyhedrons in 3-D. In one usage of the control statement ZONE all the nodes are placed in geometric zones and assigned an identifying number. This number is then addressed in the property input macro commands by specifying a $\mathrm{JA}<0$ in the definition of the loop parameters given above. For example if JA = 1 , the properties defined on the input line would be assigned to the nodes defined as belonging to geometric Group 1. The control statement ZONE may be called more than once to redefined geometric groupings.

TABLE VII. Input Description for FEHMN

Control Statement CAP (optional)

Capillary pressure model [see Eq. (29)]

Group 1- ICAP(i), cp1(i), cp2(i), cp3(i),

ICAP(i) - Relative permeability model type

$\operatorname{ICAP}(\mathrm{i})=1$, linear capillary pressure

cp1(i) - maximum capillary pressure

cp2(i) - maximum liquid saturation for capillary pressure calculation

cp3(i) - not used

Note: Only ICAP(I) $=1$ is allowed. The parameter $i$ is incremented each time a Group 1 line is read. Group 2 lines will refer to this parameter. Group 1 is ended when a line with $\operatorname{ICAP}(\mathrm{i})=0$ is encountered. 
Group 2- JA, JB,JC, ICAPT

$\mathrm{JA}, \mathrm{JB}, \mathrm{JC}$ - defined on page 60

ICAPT - reference counter for model defined in Group 1

It is recommended that macro CAP be preceded by macro RLP because capillary pressures are defined in macro RLP if van Genuchten models are employed. Care should be taken to ensure that capillary pressures are not defined twice.

Control statement COOR (required)

Group 1 - N

$\mathrm{N} \quad-$ number of nodes in the grid

Group 2 - MB, CORD1, CORD2, CORD3

MB - node number. If $\mathrm{MB}<0$ then the difference between the absolute value of $M B$ and the previously read absolute value

of $\mathrm{MB}$ is used to generate intermediate values by interpolation.

CORD1 - $\mathrm{x}$-coordinate $(\mathrm{m})$ of node $\mathrm{MB}$

CORD2 - $y$-coordinate $(m)$ of node $M B$

CORD3 - z-coordinate $(\mathrm{m})$ of node MB

NOTE: To end the control section a line with $\mathrm{MB}=0$ is entered.

Control statement COND (required)

Assign thermal conductivities of the rock [see Section I-B, Eq. (6)]

Group 1 - JA, JB, JC, THXD, THYD, THZD

JA, JB, JC - defined on page 60

THXD - thermal conductivity $(\mathrm{W} / \mathrm{mK})$ in the $\mathrm{x}$-direction

THYD - thermal conductivity $(\mathrm{W} / \mathrm{mK})$ in the $y$-direction

THZD - thermal conductivity $(\mathrm{W} / \mathrm{mK})$ in the $\mathrm{z}$-direction

Control statement CONT (optional)

Group 1 - NCNTR, CONTIM

NCNTR - time step interval for contour plots

CONTIM - time (days) interval for contour plots

NOTE: the contour data will be output whenever either of the above criteria is satisfied

Control statement CTRL (required)

Assign various control parameters needed for equation solvers and matrix solver routines (see Section I-D)

Group 1 - MAXIT, EPM, NORTH [see Eqs. (55)-(68)] 
MAXIT - maximum number of iterations allowed in either the overall Newton cycle or the inner cycle to solve for the corrections at each iteration [see Eqs. (55)-(57) and Eqs. (63)-(68)]

EPM - tolerance for Newton cycle (nonlinear equation tolerance) NORTH - number of orthogonalizations in the linear equation solver [see Eqs. (63)-(65)]

Group 2 - JA, JB, JC, IGAUS [see Eqs. (58)-(62)]

$\mathrm{JA}, \mathrm{JB}, \mathrm{JC}$ - defined on page 60

IGAUS - the order of partial Gauss elimination

Group 3 - AS, GRAV, UPWGT

AS - implicitness factor [see Eq. (40)] if $\mathrm{AS} \leq 1$, a standard pure implicit formulation will be used if $A S \geq 1$, a second-order implicit method will be used

GRAV - direction of gravity $1=\mathrm{x}$-direction, $2=\mathrm{y}$-direction, $3=z$-direction. If GRAV $>3, \mathrm{GRAV}$ is set equal to 3 . If $\mathrm{GRAV}=0$, then no gravity is used. If GRAV $\neq 0$ then a value of gravity of $9.8 \mathrm{~m} / \mathrm{s}^{2}$ is used in the code.

UPWGT - value of upstream weighting ( $0.5 \leq$ UPWGT $\leq 1.0)$ [see Sec. I-C, and the paragraph following Eq. (49)] If UPWGT $<0.5$ UPWGT is set to 0.5 If UPWGT $>1.0 \mathrm{UPWGT}$ is set to 1.0

Group 4 - IAMM, AIAA, DAYMIN, DAYMAX

IAMM - maximum number of iterations for which the code will multiply the time step size

AIAA - time step multiplier (see Sec. I-D)

DAYMIN - minimum time step size (days)

DAYMAX - maximum time step size (days)

Group 5 - ICNL, LDA

ICNL - parameter that specifies the geometry

$\begin{array}{ll}\text { if ICNL }=0 & \text { three-dimensional } \\ \text { if ICNL }=1 & \mathrm{X}-\mathrm{Y} \text { slice } \\ \text { if ICNL }=2 & \mathrm{X}-\mathrm{Z} \text { slice } \\ \text { if } \mathrm{ICNL}=3 & \mathrm{Y}-\mathrm{Z} \text { slice }\end{array}$


if $\mathrm{ICNL}=4 \quad \mathrm{X}-\mathrm{Y}$ radial slice

if $\mathrm{ICNL}=5 \quad \mathrm{X}-\mathrm{Z}$ radial slice

if $\mathrm{ICNL}=6 \quad \mathrm{Y}-\mathrm{Z}$ radial slice

LDA - parameter that specifies the external storage of geometric coefficients

LDA $=+1$, element coefficients are read in from file STOR.FE and no coefficients are calculated in the code (note: STOR.FE must be in local file space)

LDA $=0$, element coefficients are calculated in the code and not saved

$\mathrm{LDA}=-1$, element coefficients are calculated in the code and saved on file STOR.FE

Control statement DUAL (optional, see Section F)

Group 1 - JA, JB, JC, VOLFD1

$\mathrm{JA}, \mathrm{JB}, \mathrm{JC}$ - defined on page 60

VOLFD1 - volume fraction for first matrix node

Group 2 - JA, JB, JC, VOLFD2

$\mathrm{JA}, \mathrm{JB}, \mathrm{JC}$ - defined on page 60

VOLFD2 - volume fraction for second matrix node

Group 3 - JA, JB, JC, APUVD

$\mathrm{JA}, \mathrm{JB}, \mathrm{JC}$ - defined on page 60

APUVD - area per unit volume (length scale) for the matrix nodes

The quantities VOLFD1 and VOLFD2 are volume fractions and related to the total volume by

$$
\text { VOLFD1 + VOLFD2 + VOLFF }=1.0
$$

where VOLFF is the volume fraction of the fractures. The above relation must be satisified at all nodes.

Control statement ELEM (required)

Group 1 - NS, NEI,

NS - number of nodes per element

NEI - number of elements

Group 2 - MB, NELM (1), NELM (2)... NELM (NS)

MB - node number. If $\mathrm{MB}<0$ then the difference between the 
absolute value of $\mathrm{MB}$ and the previous absolute value of $\mathrm{MB}$ is used to generate intermediate values by interpolation in the code.

NELM (1) - first node of element MB

NELM (2) - second node of element MB

NELM (NS) - last node of element MB

NOTE: To end the control section a line with $\mathrm{MB}=0$ is entered.

\section{Control Statement EOS (optional)}

Equation of state, see pages 8-9)

Group 1 - IIEOSD, IPSAT, ITSAT

IIEOSD - equation of state reference number. IIEOSD $=1$ or 2 refer to high and low pressure data sets respectively in FEHMN. For these values the input in Group 2 and Group 3 will be ignored after it is entered.

IPSAT - parameter to set vapor pressure to zero. If IPSAT $\neq 0$ the vapor pressure is set to zero, otherwise the vapor pressure is calculated in the code.

ITSAT - parameter to adjust the saturation temperature. If ITSAT $<\mathbf{0}$, the saturation temperature is set to $-1000^{\circ} \mathrm{C}$. If ITSAT $>0$, the saturation temperature is set to $1000^{\circ} \mathrm{C}$.

Group 2 - EWI, EW2, EW3, EW4, EW5, EW6, EW7, EW8, EW9, EW10, EW11

EW1 - liquid reference pressure

EW2 - liquid reference temperature

EW3 - liquid reference density

EW4 - derivative of liquid density with respect to pressure at reference conditions

EW5 - derivative of liquid density with respect to temperature at reference conditions

EW6 - liquid reference enthalpy

EW7 - derivative of liquid enthalpy with respect to pressure at reference conditions

EW8 - derivative of liquid enthalpy with respect to temperature at reference conditions

EW9 - liquid reference viscosity

EW10 - derivative of liquid viscosity with respect to pressure at reference conditions 
EW11 - derivative of liquid viscosity with respect to temperature at reference conditions

Group 3 - EVI, EV2, EV3, EV4, EV5, EV6,

EV7, EV8, EV9, EV10, EV11

EV1 - vapor reference pressure

EV2 - vapor reference temperature

EV3 - vapor reference density

EV4 - derivative of vapor density with respect to pressure at reference conditions

EV5 - derivative of vapor density with respect to temperature at reference conditions

EV6 - vapor reference enthalpy

EV7 - derivative of vapor enthalpy with respect to pressure at reference conditions

EV8 - derivative of vapor enthalpy with respect to temperature at reference conditions

EV9 - vapor reference viscosity

EV10 - derivative of vapor viscosity with respect to pressure at reference conditions

EV11 - derivative of vapor viscosity with respect to temperature at reference conditions

\section{Control statement FLOW (optional)}

[see Eq. (54)]

Group 1 - JA, JB, JC, SKD, EFLOW, AIPED

$\mathrm{JA}, \mathrm{JB}, \mathrm{JC}$ - defined on page 60

SKD - heat and mass source strength $(\mathrm{kg} / \mathrm{s})$, heat only $(\mathrm{MJ} / \mathrm{s})$.

Negative value indicates injection into the rock mass.

EFLOW - enthalpy ( $\mathrm{MJ} / \mathrm{kg}$ ) of fluid injected. If the fluid is flowing from the reservoir, then the in-place enthalpy is used. If EFLOW $<0$, then $\mathrm{ABS}(\mathrm{EFLOW})$ is interpreted as a temperature and the enthal calculated accordingly. In heat only problems with EFLOW $<0$, the node is in contact with a large heat pipe that supplies heat to the node through an impedance AIPED so as to maintain its temperature near ABS (EFLOW). Large values (approximately 1000) of AIPED are recommended.

AIPED - impedance paraneter. If AIPED is nonzero, the code interprets SKD as a flowing wellbore pressure (MPa) with an impedance 
ABS(AIPED). If AIPED < 0 , flow is only allowed out of the well.

For heat only, AIPED is the thermal resistance. If $\mathrm{AIPED}=0$, SKD is flow rate.

NOTE: If the porosity of the node is zero, then there is only a temperature solution, and the code forms a source proportional to the enthalpy difference E-EFLOW, where $E$ is the in-place enthalpy and EFLOW is a specified enthalpy. The source term is given by $\mathrm{Q}=\mathrm{AIPED}(\mathrm{E}-\mathrm{EFLOW})$.

\section{Control Statement HFLX (optional)}

Group 1 - JA, JB, JC, FLUX, QFLXM

$\mathrm{JA}, \mathrm{JB}, \mathrm{JC}$ - defined on page 60

QFLUX - If QFLXM =0, then AFLUX is the heat flux (MW) if QFLXM $\neq 0$, then QFLUX is a temperature and the heat flux is calculated according to the formula: $Q_{H}=Q F L X M(T L-Q F L U X)$

QFLXM - multiplier for heat flux equation given in QFLUX description $\left(\mathrm{MW} /{ }^{\circ} \mathrm{C}\right)$

\section{Control Statement INIT (optional)}

Set initial pressure and temperature at all nodes

Group 1 - PEIN, TIN, TIN1, GRAD1, DEPTH, TIN2, GRAD2, QUAD

PEIN - initial value of pressure (MPa). If initial values are read from IREAD (see terminal input), then this value is ignored. If gravity is present, this is the value of the pressure at node 1 , and the other nodal pressures are adjusted by considering the hydraulic head.

TIN - initial value of temperature $\left({ }^{\circ} \mathrm{C}\right)$. If TIN $\leq 0$, then the initial temperatures are calculated using the pressure and temperature gradient.

NOTE: The initial temperatures are set according to the following formulas only if TIN $\leq$ 0 . Otherwise, the initial temperatures are determined from PEIN and TIN.

$$
\begin{array}{ll}
0 \leq \mathrm{Z} \leq \mathrm{DEPTH} & \mathrm{T}=\mathrm{TIN} 1+\mathrm{GRAD} 1 \times \mathrm{Z} \\
\mathrm{Z}>\mathrm{DEPTH} & \mathrm{T}=\mathrm{TIN} 2+\mathrm{GRAD} 2 \times \mathrm{Z}+\mathrm{QUAD} \times \mathrm{Z}^{2}
\end{array}
$$

TIN1 - defined in formulas above $\left({ }^{\circ} \mathrm{C}\right)$

GRAD1 - defined in formulas above $\left({ }^{\circ} \mathrm{C} / \mathrm{m}\right)$

DEPTH - defined in formulas above $(\mathrm{m})$

TIN2 - defined in formulas above $\left({ }^{\circ} \mathrm{C}\right)$

GRAD2 - defined in formulas above $\left({ }^{\circ} \mathrm{C} / \mathrm{m}\right)$ 
QUAD - defined in formulas above $\left({ }^{\circ} \mathrm{C} / \mathrm{m}^{2}\right)$

Control statement ITER (optional, see defaults below)

NOTE: If the user is not familiar with the linear equation solver routines in FEHMN please leave out control statement ITER and associated lines (see Section I-D).

Group 1 - G1, G2, G3, TMCH, OVER

G1 - multiplier for the linear convergence region of the Newton-Raphson iteration

G2 - multiplier for the quadratic convergence region of the NewtonRaphson iteration

G3 - tolerance for the adaptive implicit method (multiplying factor for Newton-Raphson tolerance)

TMCH - machine tolerance. If satisfied by the residual norm, the Newton iteration is complete

OVERF - over relaxation factor for passive nodes in adaptive implicit method

Group 2 - IRDOF, ISLORD, IBACK, ICOUPL, RNMAX IRDOF - enables the reduced degree of freedom method ISLOR: ; - reordering parameter. The ordering can be understood by labeling the mass equation as 1 , the heat equation as 2 , and the $\mathrm{CO}_{2}$ equation (if it exists) as 3 . The value of ISLORD and the corresponding equation order is given below. ISLORD Mass, Heat Mass, Heat, $\mathrm{CO}_{2}$

$\begin{array}{lll}0 & 1,2 & 1,2,3 \\ 1 & 2,1 & 1,3,2 \\ 2 & & 2,1,3 \\ 3 & & 2,3,1 \\ 4 & & 3,1,2 \\ 5 & & 3,2,1\end{array}$

the ordering has an effect on the speed of convergence of several solution algorithms, but will not affect most users

IBACK - back substitution parameter

ICOUPL - number of SOR iteration used in reduced degree of freedom methods

RNMAX - maximum running time for problem before the solution is stopped

If control statement ITER is not present the following default values are used G1 $=0.001$, 
$\mathrm{G} 2=0.0001, \mathrm{G} 3=0.0001, \mathrm{TMCH}=10^{-8}, \mathrm{OVERF}=0.0, \mathrm{IRDOF}=0$.

Control statement NGAS (optional)

Noncondensable gas transport

Group 1 - ICO2D

ICO2D - solution descriptor for noncondensible gas transport

ICO2D - 0 data from control group NGAS is read but not used

ICO2D - 1, 2, reduced degree of freedom solver technology is used

ICO2D - 3, full 3 degrees of freedom solution water/noncondensible gas solution

Group 2 - JA, JB, JC, PC02

JA, JB, JC - defined as in control statement ROCK

$\mathrm{PC} 02$ - initial partial pressure of non-condensible gas. If $\mathrm{PCO} 2<0$ then $\mathrm{ABS}(\mathrm{PCO} 2)$ is interpreted as a temperature and the partial pressure of the noncondensible gas is calculated according to the formula: $P C O 2=P_{T}-P_{S A T}(T)$ where $P_{T}$ is the total pressure and $P_{S A T}(T)$ is the water saturation pressure and is a function of temperature only.

Group 3 - JA,

$\mathrm{JB}$,

$\mathrm{JC}$,

CPNK

$\mathrm{JA}, \mathrm{JB}, \mathrm{JC}$ - defined on page 60

CPNK - injection concentration of noncondensible gas for injection mode $(\mathrm{SK}(\mathrm{i})<0)$. If it is a production node $(\mathrm{SK}(\mathrm{i})>0)$ then the in place concentration is used.

Note: For compatability with older versions the user may substitute "CO2I" for "NGAS" in the input file.

Control statement NODE (required)

Specify the node numbers for which detailed printed output is desired. The plotting postprocessor FEHPLTR will also use these nodes.

Group 1 - M

M - number of nodes for which information will be printed on IOUT (see terminal input). If $M<0$, pressure and temperature will be written for all nodes. 
Group 2 - MN (1), MN (2) ... MN (M)

MN - node numbers for which information will be printed on IOUT, $M$ nodes (see Group 1).

If $\mathrm{NN}(\mathrm{I})<0$, then coordinates are used to define the print-out node. The coordinate sets $(x, y, z)$ for each $N M(I)<0$ are added after Group 2. For 2-D problems put $z=0$.

Control statement PERM (required)

Assign absolute permeabilities of the rock [see section I-B, Eq. (7)-(8)]

Group 1 - JA, JB, JC, PNXD, PNYD, PNZD

$\mathrm{JA}, \mathrm{JB}, \mathrm{JC}$ - defined on page 60

PNXD - permeability in the $x$-direction $\left(\mathrm{m}^{2}\right)$

PNYD - permeability in the y-direction $\left(\mathrm{m}^{2}\right)$

PNZD - permeability in the $z$-direction $\left(\mathrm{m}^{2}\right)$

Control Statement PPOR (Optional, default is IPOROS=0)

For variable porosity/permeability problems, define model type (see Section I-B)

Group 1 - IPOROS, R1, R2, R3

IPOROS - Porosity/permeability type [see Eqs. (39)-(42)]

$=0$ constant porosity

$=1$ simple linear model

$=2$ Gangi stress model

$\mathrm{R} 1, \mathrm{R} 2, \mathrm{R3}$ - parameters used in the various models

Group 2 - JA, JB, JC, R4, R5

$\mathrm{JA}, \mathrm{JB}, \mathrm{JC}$ - defined on page 60

$\mathrm{R} 4$ - variable parameter used in porosity/permeability models

R5 - variable parameter used in porosity/permeability models

\begin{tabular}{ccll} 
& \multicolumn{2}{c}{ Model } \\
\cline { 1 - 1 } Parameter & & Linear (Eq. 39) & Gangi(Eq. 40-41) \\
R1 & & $\mathrm{C}_{r}$ & $\propto$ \\
R2 & & $\mathrm{C}_{g}$ & $\mathrm{E}$ \\
R3 & & not used & $\sigma$ \\
R4 & & not used & m(exponent) \\
R5 & & not used & $\mathrm{P}_{o}$
\end{tabular}

For the linear model $P_{o}$ is PEIN in macro INIT. $\phi_{o}$ is PSD in macro ROCK and $k_{o}$ is from macro PERM for both models. 
Control statement PRES (optional)

Assign nonuniform initial pressure and temperature values

Group 1 - JA, JB, JC, PHRD, TIND, IEOSD

$$
\begin{aligned}
& \mathrm{JA}, \mathrm{JB}, \mathrm{JC} \text { - defined on page } 60 \\
& \text { PHRD - initial pressure (MPa) } \\
& \text { TIND - initial temperature (If IEOSD }=1 \text { or } 3 \text { ) or initial } \\
& \text { saturation (if IEOSD }=2 \text { ) } \\
& \text { IEOSD - thermodynamic region parameter if IEOSD }=1 \text {, then } \\
& \text { in compressed liquid region, if IEOSD }=2 \text {, the } \\
& \text { saturation region, if IEOSD }=3 \text {, the superheated region. }
\end{aligned}
$$

NOTE: The initial values defined in control statement PRES supersede all others.

Control statement RLP (optional)

Relative permeability models [see Eqs. (25)-(27)]

Group 1 - IRLP(i), RP1, RP2, RP3, RP4

IRLP - Relative permeability model type

$\operatorname{IRLP}(\mathrm{i})=1$ linear type

RP1 - irreducible liquid saturation

RP2 - irreducible vapor saturation

RP3 - maximum liquid saturation

RP4 - maximum vapor saturation

$\operatorname{IRLP}(\mathrm{i})=2$ Corey type

RP1 - irreducible liquid saturation

RP2 - irreducible vapor saturation

RP3 - not used

RP4 - not used

$\operatorname{IRLP}(\mathrm{i})=3$ van Genuchten (note different input)

NOTE: With a van Genutchen formulation (IRLP $=3$, IRLP $=4$ ), both the relative permeability and capillary information are input. Therefore there is no capillary pressure input and information should not be input in the capillary pressure section (macro CAP) for those nodes.

RP1, RP2, RP3, RP4, RP5, RP6

$R P 1$ - residual liquid saturation

RP2 - maximum liquid saturation

RP3 - $\alpha$, parameter for model

RP4 - $\beta$ parameter for model

RP5 - maximum capillary pressure 
RP6 - fractional difference in pressure at maximum and cutoff saturation IRLP(i) $=4$ combined van Genutchen model. All the values for IRLP $=3$ are read in plus the following RP7, RP8, RP9, RP10, RP11, RP12, RP13,

RP14, RP15

RP7 - residual liquid saturation for fracture

RP8 - maximum liquid saturation for fracture

RP9 - $\alpha$, for fractures

RP10 - $\beta$, for fractures

RP11 - maximum capillary pressure for fracture

RP12 - fractional difference in pressure at maximum and cutoff saturation

$R P 13$ - fracture intrinsic permeability $\left(\mathrm{m}^{2}\right)$

RP14 - matrix intrinsic permeability $\left(\mathrm{m}^{2}\right)$

RP15 - fracture porosity

Group 1 is ended when a line with $\operatorname{IRLP}(i)=0$ is encountered.

Group 2 - JA, JB, JC, I

$\mathrm{JA}, \mathrm{JB}, \mathrm{JC}$ - defined on page 60

I - number referring to the sequence of models read in Group1

NOTE: The parameter $i$ is incremented each time a Group 1 line is read. Group 2 lines will refer to this parameter.

Control statement ROCK (required)

Assign rock density, specific heat and porosity [see Section I-B, Eqs. (1)-(10)]

Group 1 - JA, JB, JC, DENRD, CPRD, PSD

$\mathrm{JA}, \mathrm{JB}, \mathrm{JC}$ - defined on page 60

DENRD - rock density $\left(\mathrm{kg} / \mathrm{m}^{3}\right)$

CPRD - rock specific heat $\left(\mathrm{MJ} / \mathrm{kg} /{ }^{\circ} \mathrm{C}\right)$. If CPRD $>1$ the code will assume the units are $\mathrm{J} / \mathrm{kg} /{ }^{\circ} \mathrm{C}$ and multiply by $10^{-6}$

PSD - porosity

Control Statement SOL (required)

Group 1 - NTT, INTG

NTT - parameter that defines the type of solution required if NTT $\geq 0$ heat and mass transfer solution if NTT $<0$ heat transfer solution

INTG - parameter that defines element integration type (refer to Section I-B) if INTG $\leq 0$ Lobatto quadrature is used, recommended for heat and mass problems without stress. 
if INTG > 0 Gauss quadrature is used, recommended for problems requiring a stress solution.

\section{Control Statement STEA}

No input is associated with this macro statement. This statement enables a 1-D solution in the $\mathrm{y}$-direction (2-D) or $\mathrm{z}$-direction (3-D) when gravity is present to generate an initial steady state solution.

Control Statement STOP (required)

NOTE: No input is associated with this control statement. It signals the end of input, and as such it always appears as the last line of an input deck.

\section{Control Statement TEXT}

Following the control statement, text is input until a blank line is inserted to signal the end of the control statement. This text is printed on the output file.

\section{Control statement TIME (required)}

Group 1 - DAY, TIMS, NSTEP, II, YEAR, MONTH

DAY - initial time step size (days)

TIMS - simulation maximum time (days)

NSTEP - maximum number of time steps allowed

II - print-out interval for nodal information (pressure, enthalpy etc.), as set up under control statement node.

YEAR - year that simulation starts

MONTH - month that simulation starts

Group 2 - DIT1, DIT2, DIT3, ITC

DIT1 - time (days) for time step change

DIT2 - new time step size (days). If DIT2 $<0$ then ABS (DIT2) is the new time step multiplier

DIT3 - implicitness factor for new time step (use $\leq 1.0$ backward Euler, $>1.0$ for second-order implicit scheme).

ITC - new print-out interval

NOTE: The code proceeds to the next control statement when a line is encountered with DIT1 $=0$. A contour plot will be drawn at each DIT1. A restart file will be written at each DIT1. 
Control statement TRAC (optional)

[see Eqs. (16)-(19) and (22)-(24) and Table II]

Group 1 - ANO, AWC, EPC, UPWGTA

ANO - initial tracer concentration

AWC - Implicitness factor for tracers. AWC $>1.0$ gives 2nd order solution; $\mathrm{AWC} \leq 1.0$ gives 1 st order solution

EPC - equation tolerance for tracer solution

UPWGTA - upstream weighting term for the tracer solution

If UPWGT < 0.5 UPWGTA is set to 0.5

If UPWGTA > 1.0 UPWGTA is set to 1.0

Group 2 - DAYCS, DAYCF, DAYHF, DAYHS

DAYCS - time which the tracer solution is enabled

DAYCF - time which the tracer solution is disabled

DAYHF - time which the heat and mass transfer solution is disabled

DAYHS - time which the heat and mass transfer solution is enabled

Group 3 - IACCMX, DAYCM, DAYCMM, DAYCMX

IACCMS - maximum number of iterations allowed in tracer solution if time step multiplier is enabled

DAYCM - time step multiplier for tracer solution

DAYCMM - minimum time step for tracer solution

DAYCMX - maximum time step for tracer solution

Group 4 - NSPECIES

NSPECIES - number of different tracers

NOTE: TRAC groups $5,6,7$, and 8 are entered as a unit for each different tracer.

Group 5 - ICNS, IADSF, AIADSF, A2ADSF, BETAD

ICNS - phase designation. IF ICNS $\leq 0$ a gas phase tracer is used.

If ICNS $>0$, then a liquid phase tracer is used.

IADSF - designates tracer type [see Eq. (23)-(24) and Table II]

$=0$, refers to the conservative tracer

$=1$, refers to the linear model

$=2$, refers to the Freundlich model

$=3$, refers to the modified Freundlich model

$=4$, refers to the Langmuir model

A1ADSF - $\alpha_{1}$ parameter

A2ADSF - $\alpha_{2}$ parameter

BETAD - $\beta$ parameter 
Group 6 - ICRATE, A1R, A2R, A3R, A4R

ICRATE - designates reaction model type see Eq. (24).

A1R - parameter in rate model

A2R - parameter in rate model

A3R - parameter in rate model

A4R - parameter in rate model

ICRATE $=1$ refers to the simple model (p. 9) and ICRATE $\geq 2$ refers to the more complicated model (p. 9).

Group 7 - JA, JB, JC, TCXD, TCYD, TCZD

$\mathrm{JA}, \mathrm{JB}, \mathrm{JC}$ - defined on page 60

TCXD - dispersivity coefficient in the $\mathrm{x}$-direction $\left(\mathrm{m}^{2} / \mathrm{s}\right)$

TCYD - dispersivity coefficient in the y-direction $\left(\mathrm{m}^{2} / \mathrm{s}\right)$

TCZD - dispersivity coefficient in the $\mathrm{z}$-direction $\left(\mathrm{m}^{2} / \mathrm{s}\right)$

Group 8 - JA, JB, JC, ANQO

$\mathrm{JA}, \mathrm{JB}, \mathrm{JC}$ - defined on page 60

ANQO - initial concentration of tracer, which will supersede the value given by ANO in Group 1. Note that if initial values of pressure and enthalpy are read from a file, then the code will aiso read initial values for the tracer from the file.

Group 9 - JA, JB, JC, CNSK, T1SK, T2SK

$\mathrm{JA}, \mathrm{JB}, \mathrm{JC}$ - defined on page 60

CNSK - injection concentration of injection node. If it is a production node, then the in-place concentration is used

T1SK - time (days) when tracer injection begins

T2SK - time (days) when tracer injection ends

NOTE: Injection nodes must be specified in control statement flow.

Control statement ZONE (Optional, default is input by nodes)

Group 1- IZONE

IZONE - identification number for geometric input

Group 2- X1, X2, X3, X4, Y1, Y2, Y3, Y4 (for 2-D problems)

$\mathrm{X} 1-\mathrm{X} 4$ - $\mathrm{X}$ coordinates for zone IZONE

$\mathrm{Y} 1-\mathrm{X} 4$ - $\mathrm{Y}$ coordinates for zone IZONE

$\mathrm{X} 1-\mathrm{X} 8, \mathrm{Y} 1-\mathrm{Y} 8, \mathrm{Z} 1-\mathrm{Z} 8$ (for 3-D problems)

$\mathrm{X} 1-\mathrm{X} 8$ - $\mathrm{X}$ coordinates for zone IZONE

Y1-Y8 - Y coordinates for zone IZONE

Z1-Z8 - Z coordinates for zone IZONE 
NOTE: If the first four characters in Group 2 are 'LIST' then the code reads a list of $x, y$, z-coordinates, one set perline until a blank line is encountered. The nodes corresponding to these coordinates make up the zone. The geometric zone description is implemented by defining geometric regions. The coordinates given in Group 2 are defined in Fig. 5. All properties defined by node (JA, JB, JC) may be defined by ZONE. In the previous macro descriptions if JA $<0$, then the zone IZONE $=$ ABS (JA) is referenced. The macro ZONE must precede the usage of a ZONE reference. ZONE can be called more than once.

\section{Graphics Postprocessing}

Two graphics postprocessing codes are described in this section. They are written using the DISSPLA graphics package. Except for the plot area specification only the primitive line drawing commands are used. Therefore the routines should be easily convertible to other systems. A capability for time history plots for variables (FEHPLTR) and contour plotting (FECPLTR) are provided. Though these codes have not been verified, users may use them at their convenience.

1. FEHPLTR is a postprocessor program for FEHMN. It uses output information from FEHMN in the file FE.HIS. The program organizes information found in the file FE.HIS for plotting time histories of the variables temperature, pressure, enthalpy, flow rate, concentration, and capillary pressure. The user input is found in file HIS.INS.

Group 1 - HEADER - 80 character title

Group 2 - NNODE - number of input nodes. This must correspond to the number of print-out nodes specified in the input file for FEHMN.

Group 3 - ITYPE, IPLOT (I), II=1, NNODE

ITYPE - variable designator

$=1$, enthalpy plot

$=2$, flow rate plot

$=3$, temperature plot

$=4$, pressure plot

$=5$, capillary pressure plot

$=6$, concentration plot

IPLOT(I) - designates if the Ith node is to be plotted on the current plot. IPLOT(I) $\neq 0$ then the Ith node will be plotted.

IPLOT(I) $=0$ then the Ith node will not be plotted. 
Group 4- A - parameter to scale the plot variable according to the formula $P *=\mathrm{A}(\mathrm{P}-\mathrm{B})$

B - parameter to scale the plot variable according to the formula given in the last description

C - parameter to scale the time variable according to the formula $t *=C(T-D)$

D - parameter to scale the time variable according to the formula given in this last description

Note: If $0,0,0,0$ is entered, no action is taken (i.e., $P=p$ )

Group 5 - XLEN, YLEN

XLEN - physical plot horizontal dimension in inches

YLEN - physical plot vertical dimension in inches

Group 6 - AXIS

AXIS

- plot type

AXIS $<0$ time axis is a $\log$ axis and the variable axis is linear

AXIS $=0$, both axes are linear

AXIS $>0$, both axes are log scales

Group 7 - DAYMIN, DAYMAX, DAYTIC, NTIC

DAYMIN - minimum limit for time axis

DAYMAX - maximum limit for time axis

DAYTIC - increment for time axis

NTIC - tic marks per increment for the time axis

Group 8 - VMIN, VMAX, VTIC, NIY

VMIN - minimum limit for variable axis

VMAX - maximum limit for variable axis

VTIC - increment value for variable axis

NIY - tic marks per interval for the variable axis

Group 9 - TITLE

TITLE - 30 character ( $\max$ ) heading for top of plot

Group 10 - TITLX

TITLX - 30 character ( $\max$ ) heading for time axis 
TITLY - 30 character ( $\max$ ) heading for the variable axis

Group 12 is entered only if ITYPE $=6$ is chosen and a concentration plot is generated. Since several (up to 10) concentration profiles can be plotted, the species number to be plotted must be specified. Group 12 provides this information.

$$
\begin{aligned}
& \text { Group } 12-[\text { ISPT (II) II }=1, \text { NSPECI }] \\
& \begin{aligned}
\text { ISPT(II) - } & \text { plot designator for the IIth specie } \\
& \neq 0 \text { then that specie is plotted } \\
& =0 \text { then that specie is not plotted }
\end{aligned}
\end{aligned}
$$

NOTE: the variable NSPECI is obtained by the code from the file FE.HIS. Groups 3-12 may be repeated for additional plots.

2. FECPLTR. The computer code FECPLTR is the postprocessor code for FEHMN which makes contour plots. Its input file is the unformatted file FE.CON generated by FEHMN. The plot instruction commands are given in the file CON.INS. The input lines are described below and are in free format except where noted.

Group 1 - ICHOS

ICHOS - dimensionality parameter

$=2,2$ dimensional contour plots are generated

$\neq 2,3$-dimensional contour plot information is provided

NOTE: the form of the input that follows is not implemented as yet for the value of ICHOS $=3$.

Group 2 - HEADER

Header - title ( 80 characters maximum)

Group 3 - NCI, IGRID

NCI - contour label interval NCI $=0$, no labels are drawn on contours, contours are given different line types (dash, solid, etc.) and identified on the side of the plot NCI $\neq 0$, the contours are labeled on the contour line

IGRID - grid parameter

$=0$, no grid drawn with contours

$\neq 0$, grid is drawn with contours

Group 4- AX, BX, AY, BY

AX - minimum $x$ coordinate for problem $(m)$

BX - maximum $x$ coordinate for problem $(m)$ 
AY - minimum y coordinate for problem (m)

BY - maximum y coordinate for problem (m)

Group 5- VLENM, VRMAX

VLENM - length of maximum vector (in grid dimensions)

VRMAX - size of maximum vector $(\mathrm{m} / \mathrm{s})$

Group 6- XWIN, YWIN

XWIN - physical size of the $x$ direction of plot window (in)

YWIN - physical size of the $y$ direction of plot window (in)

Group 7- IVLP, CNTRT, CNTRP, CNTRC, SCALE

IVLP - plot phase parameter IVLP $<0$, vapor phase only plots will be drawn. IVLP $>0$, liquid phase only plots will be drawn

CNTRT - contour interval for temperature (liquid region) or saturation (vapor region)

CNTRP - phase pressure contour interval

CNTRC - phase contour interval for species transport

SCALE - scaling parameter for velocity vectors. No vectors will be plotted for vectors of magnitude less than VRMAX * SCALE (see Group 5).

Note: If CNTRT, CNTRP, CNTRC are $<0$, then the contour values are provided in a user supplied list. (Group 8 is used.) If they are $>0$ then Group 8 is not used.

Group 8 - NV, (CVAL(I), I = 1, NV)

NV - number of contour values

CVAL(I) - contour value (can be temperature, saturation pressure, or concentration

If multiple contour piots are desired, the user may provide additional data sets for each plot (i.e., Groups 1-7). Alternatively, the user may specify the same parameter set for each plot by not providing additional sets in the data file CON.INS. This way all the plot sets output by FEHMN and in FE.CON will be plotted. 


\section{Example Input Files And Output Files}

This section contains three example problem runs with FEHMN. Input files and output files are provided. Other examples may be found in the FEHMN software verification report (Zyvoloski and Dash, 1991).

1. Heat Conduction. This 3-D problem demonstrates the code performance on a purely conductive problem. It is useful in showing the numerical accuracy of the finite elements and providing the user with an example of 3-D input. While we give only the $4 \times 4 \times 4$ mesh input and output, accuracy results are presented for the $4 \times 4 \times 4,8 \times 8 \times 8$, and $12 \times 12 \times 12$ meshes. Table VIII shows the parameters used in the computer runs. Figure 8 depicts the problem geometry (due to symmetry only a quarter of the cube is depicted). Basically the cube is initially at $200^{\circ} \mathrm{C}$ and a boundary condition of $100^{\circ} \mathrm{C}$ is applied on all sides. Table IX gives the coordinate positions for the various error sampling points as well as defining the runs. As can be seen from Table $\mathrm{X}$ even the crudest grid produced errors of less than $9 \%$. Other grids produced much better results. For the $4 \times 4 \times 4$ grid, the input to the preprocessor GENMSH is shown in Fig. 9. The plot produced by GENMSH is given in Fig. 10. The input file for FEHMN is provided in Fig. 11. It is worth noting he:e that the part of the file from the macro command COOR to the end was produced by GENMSH (file GEOM.DAT) and appended to the other macro commands with a text editor. Figure 12 gives the output for the $4 \times 4 \times 4$ run. The user

TABLE VIII. Input Parameters for the 3-D Heat Conduction Problem

Parameter

Rock thermal diffusivity

Rock thermal conductivity

Rock density

Rock specific heat

Width

Length

Depth

Initial temperature

Surface temperature

for all $x, y, z=0.5 \mathrm{~m}$
Symbol

Value

$\begin{array}{cl}{ }^{\star} \kappa & \\ \kappa_{r} & 2.7 \mathrm{~W} / \mathrm{m} \mathrm{K} \\ \rho_{r} & 2700 \mathrm{~kg} / \mathrm{m}^{3} \\ C_{r} & 1000 \mathrm{~J} / \mathrm{kgK} \\ \mathrm{a} & 0.5 \mathrm{~m} \\ \mathrm{~b} & 0.5 \mathrm{~m} \\ \mathrm{c} & 0.5 \mathrm{~m} \\ T_{0} & 200^{\circ} \mathrm{C} \\ T_{s} & 100^{\circ} \mathrm{C}\end{array}$

${ }^{\star} \kappa=\frac{\kappa_{r}}{\rho_{r} C_{r}}$ 
TABLE IX. Sample Problems for 3-D Heat Conduction

Problem

1
2
3
4
5

3-D Mesh Elements
$4 \times 4 \times 4$ brick
$8 \times 8 \times 8$ brick
$12 \times 12 \times 12$ brick
$8 \times 8 \times 8$ prism
$8 \times 8 \times 8$ mixed
brick and prism

\begin{tabular}{ll}
\hline Comparison & $\mathrm{x}=0.000 \mathrm{y}=0.000 \mathrm{z}=0.000$ \\
coordinate & $\mathrm{x}=0.000 \mathrm{y}=0.125 \mathrm{z}=0.250$ \\
positions & $\mathrm{x}=0.125 \mathrm{y}=0.250 \mathrm{z}=0.375$ \\
& $\mathrm{x}=0.375 \mathrm{y}=0.375 \mathrm{z}=0.375$
\end{tabular}

TABLE X. Comparison of Analytical and Model Results for 3-D Heat Conduction

Maximum Error (\%)

Coordinate position

\begin{tabular}{lllll}
\hline 1 & 2 & 3 & 4 & 5 \\
\hline
\end{tabular}

$\begin{array}{lllllll}\mathrm{x}=0.000 \mathrm{y}=0.000 \mathrm{z}=0.000 & 2.139 & 0.710 & 0.427 & 0.718 & 0.570 \\ \mathrm{x}=0.000 \mathrm{y}=0.125 \mathrm{z}=0.250 & 1.784 & 0.572 & 0.339 & 0.560 & 0.572 \\ \mathrm{x}=0.125 \mathrm{y}=0.250 \mathrm{z}=0.375 & 3.546 & 1.382 & 0.862 & 1.372 & 1.382 \\ \mathrm{x}=0.375 \mathrm{y}=0.375 \mathrm{z}=0.375 & 8.517 & 3.544 & 2.301 & 3.379 & 3.544\end{array}$


instruction file for the postprocessor FEHPLTR is shown in Fig. 13. The plot produced by FEHPLTR is given in Fig. 14.

2. Toronyi Two-Phase Example. This problem is a model of a two-phase, highly permeable geothermal reservoir originally proposed by Toronyi and Farouq Ali (1977) and solved by a number of authors (Mercer and Faust 1975, Thomas and Pierson 1978). The model reservoir is shown in Fig. 15. The input for the problem is presented in Table XI. As with Thomas and Pierson, time steps of 10 days with an initial time step of 8.3 days were used. The final state corresponds to $19 \%$ of the original water mass removed. Figure 15 also shows the results obtained in this study using quadrilateral elements compared with the results obtained by Thomas and Pierson. Good agreement is evident. This is somewhat surprising considering the elements have a length/width ratio of 10. The input for the GENMSH preprocessor is shown in Fig. 16. The input file is given in Fig. 17. The part of the input file from the macro COOR was generated by GENMSH and inserted in the input file using a text editor. Output for the first computer run is shown in Fig. 18.

TABLE XI. Parameters for Toronyi Example

Parameter

Symbol

Value

$\begin{array}{lcl}\text { Reservoir permeabilty } & \mathrm{k} & 9.869 \times 10^{-13} \mathrm{~m}^{2} \\ \text { Reservoir porosity } & \phi & 0.05 \\ \text { Rock thermal conductivity } & \kappa_{r} & 1.73 \mathrm{~W} / \mathrm{m} \cdot \mathrm{K} \\ \text { Rock density } & \rho_{r} & 2500 \mathrm{~kg} / \mathrm{m}^{3} \\ \text { Rock specific heat } & C_{r} & 1000 \mathrm{~J} / \mathrm{kg} \cdot \mathrm{K} \\ \text { Aquifer length } & - & 1828 \mathrm{~m} \\ \text { Aquifer width } & - & 182.8 \mathrm{~m} \\ \text { Initial water saturation } & s_{i j}^{0} & 0.2 \\ \text { Aquifer discharge } & q_{m} & 0.05 \mathrm{~kg} / \mathrm{m} \cdot \mathrm{s} \\ \text { Initial Pressure } & P_{i j}^{0} & 4.4816 \mathrm{MPa}\end{array}$

3. DOE Code Comparison Project, Problem 5, Case A. This problem involves multiphase flow in a 2-D horizontal reservoir. The problem is characterized by a moving two-phase region, i.e., the fluid produced at the production well is replaced by cold water recharge over one of the outer boundaries. The problem parameters are given in Table XII and the geometry and boundary conditions are shown in Fig. 19. Of particular note is the variable initial temperature field and the prescribed pressure and temperature boundary. This problem shows that two-phase coding as well as the phase change algorithm are working properly, since numerical difficulties can occur as nodes go from two-phase fluid to compressed water.

There is no analytical solution for this problem, but six researchers produced results for the DOE code comparison project (Molloy, 1980). The reader is referred to this reference for a more detailed discussion of this problem and the code comparison. Results from 
this problem are compared to those from the other codes, obtained from Molloy (1980) as a check on FEHMN. The results for the outlet temperature, shown in Fig. 20, are in excellent agreement with the other codes. The results for the outlet pressure, Fig. 21, and pressure at an observation well $125 \mathrm{~m}$ distant, Fig. 22 are also in good agreement with the other codes.

The input for the preprocessor GENMSH is given in Fig. 23. The input to FEHMN is given in Fig. 24. The printout for the computer is provided in Fig. 25. The part of the input file from the macro COOR was generated by GENMSH. A contour plot of temperature was also generated for this problem. The user generated input file CON.INS is shown in Fig. 26. The contour plot is given in Fig. 27.

TABLE XII. Input Parameters for the DOE Code Comparison Project, Problem 5, Case A

Parameter

Reservoir permeabilty

Reservoir porosity

Rock thermal conductivity

Rock density

Rock specific heat

Reservoir length

Reservoir thickness

Liquid residual saturation

Gas residual saturation

Reservoir discharge

Initial Pressure
Symbot

Value
Production well coordinates:

Observation well coordinates:

Initial temperature distribution:

$\begin{array}{cl}\mathrm{k} & 2.5 \times 10^{-14} \mathrm{~m}^{2} \\ \phi & 0.35 \\ \kappa_{r} & 1 \mathrm{~W} / \mathrm{m} \cdot \mathrm{K} \\ \rho_{r} & 2500 \mathrm{~kg} / \mathrm{m}^{3} \\ C_{r} & 1000 \mathrm{~J} / \mathrm{kg} \cdot{ }^{\circ} \mathrm{C} \\ x & 300 \mathrm{~m} \\ y & 200 \mathrm{~m} \\ s_{l r} & 0.3 \\ s_{g r} & 0.1 \\ q_{m} & 0.05 \mathrm{~kg} / \mathrm{m} \cdot \mathrm{s}) \\ P_{o} & 3.6 \mathrm{MPa}\end{array}$

$\mathrm{x}=62.5 \mathrm{~m}, \mathrm{y}=62.5 \mathrm{~m}$

$\mathrm{x}=162.5 \mathrm{~m}, \mathrm{y}=137.5 \mathrm{~m}$

$$
T(x, y, 0)=\left(\begin{array}{l}
240 \\
240-160 \\
160
\end{array}\left[\frac{r-100}{200}\right]^{2}+80\left[\frac{r-100}{200}\right]^{4}\right){ }^{\circ} C \quad \begin{array}{r}
r \leq 100 m \\
100<r<300 m \\
r \geq 300 m
\end{array}
$$

where $r=\sqrt{x^{2}+y^{2}}$ 


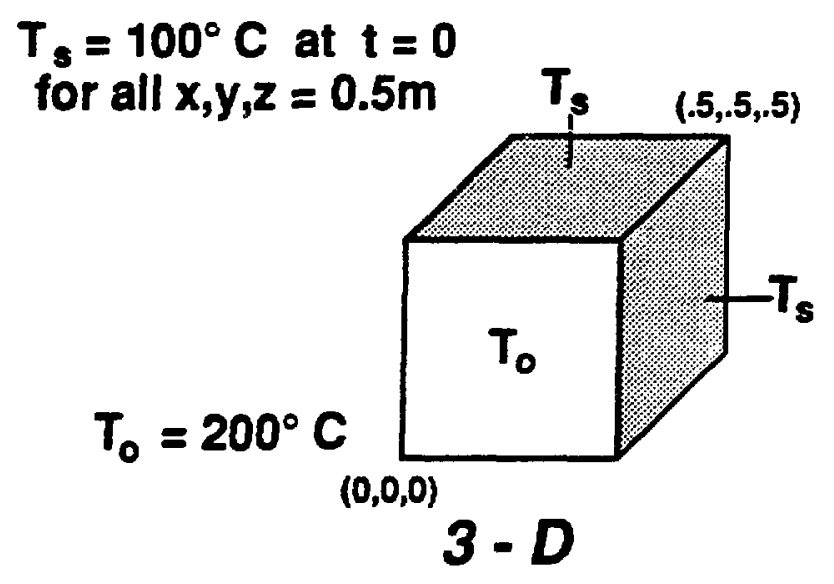

Fig. 8. Schematic diagram of the 3-D heat conduction problem.

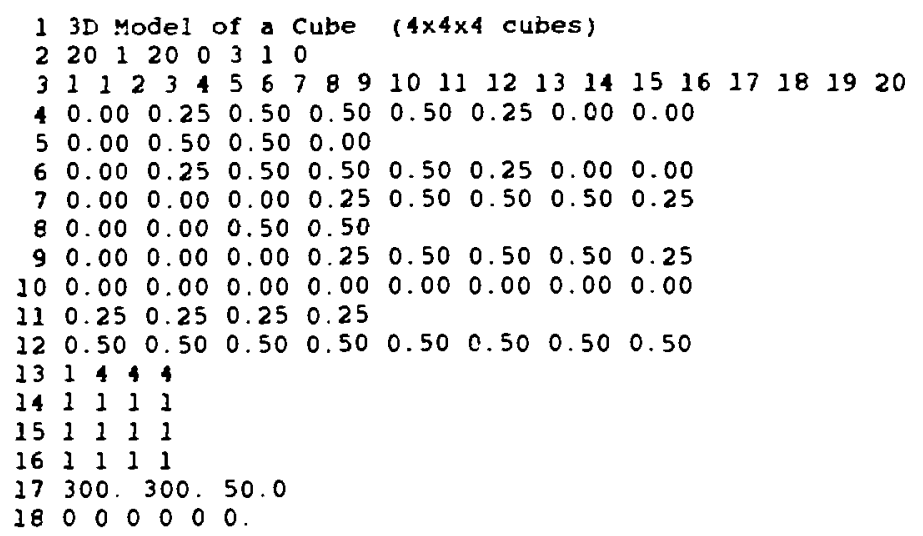

Fig. 9. Input for preprocessor GENMSH for 3-D heat conduction example. 
30 Model of a Cube $(4 \times 4 \times 4$ cubes)

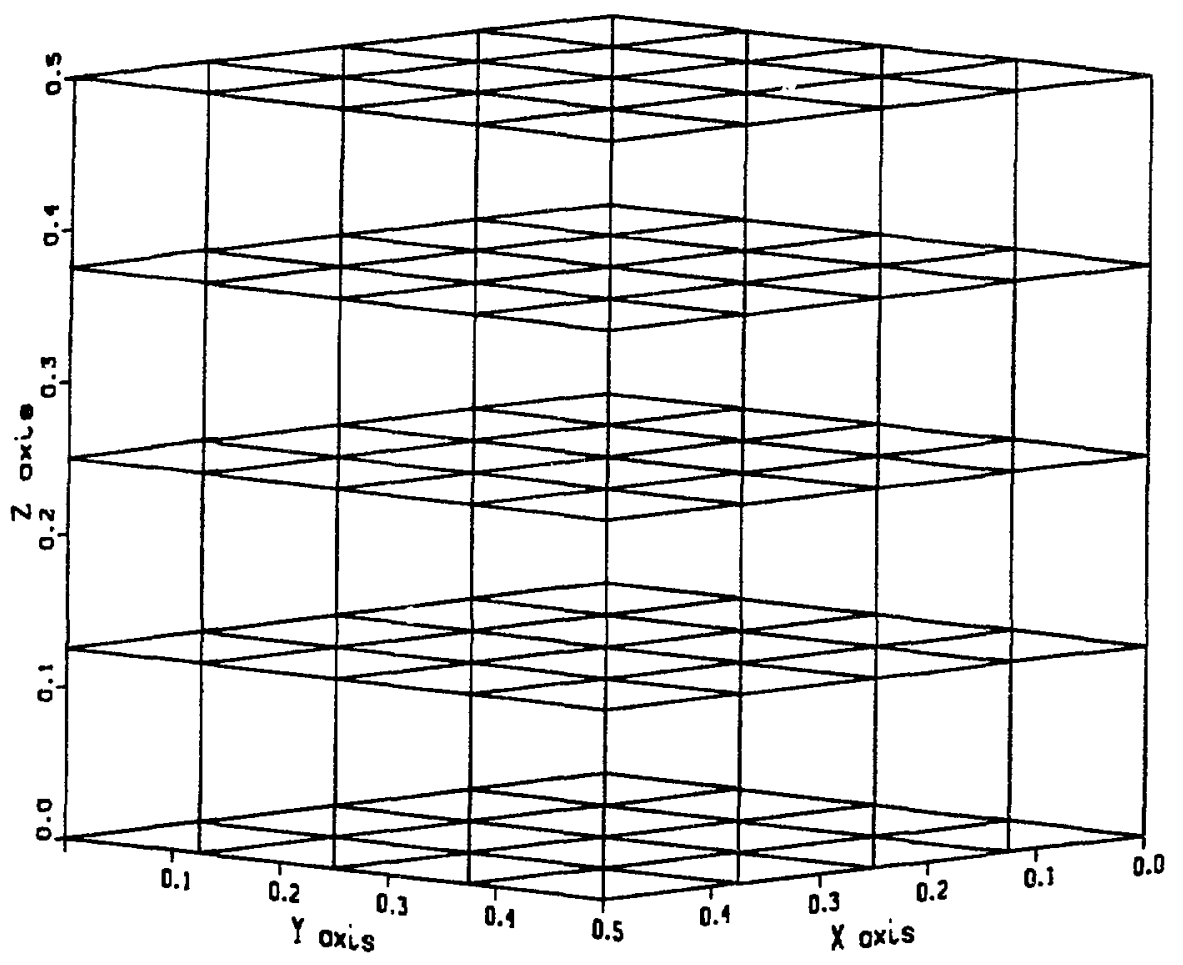

Fig. 10. Grid for $4 \times 4 \times 4$ heat conduction problem. 
$\star \star \star \star \star \star 3-D$ Heat Conduction Mode1 ( $4 \times 4 \times 4$ cubes)

node

8

rlp

$\begin{array}{llll}001 & 056 & 087 & 094\end{array}$

$\begin{array}{llll}005 & 025 & 002 & 125\end{array}$

20.0 .0 .0$.

0

$\begin{array}{llll}1 & 125 & 1 & 1\end{array}$

$0 \begin{array}{llll}0 & 0 & 0 & 0\end{array}$

sol

$-1 \quad-1$

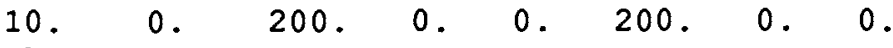

rock

$\begin{array}{rrrrlll}1 & 125 & 1 & 2700 . & 1000 . & 0 . & 1.0 \\ 0 & 0 & 0 & 0 . & 0 . & 0 . & 0.0\end{array}$

cond

$\begin{array}{rrrlll}1 & 125 & 1 & 2.7 e-00 & 2.7 e-00 & 2.7 e-00 \\ 0 & 0 & 0 & 0 . & 0 . & 0 .\end{array}$

perm

$1125 \quad 1 \quad 1 . e-30 \quad 1 . e-30 \quad 1 . e-30 \quad 0.0 .0$.

flow

$\begin{array}{llllll}101 & 125 & 1 & 10.00 & -100.00 & 1 . e 03\end{array}$

$\begin{array}{llllll}5 & 125 & 25 & 10.00 & -100.00 & 1 . e 03\end{array}$

$\begin{array}{llllll}10 & 125 & 25 & 10.00 & -100.00 & 1 . \mathrm{e} 03\end{array}$

$\begin{array}{llllll}15 & 125 & 25 & 10.00 & -100.00 & 1.003\end{array}$

$\begin{array}{llllll}20 & 125 & 25 & 10.00 & -100.00 & 1 . e 03\end{array}$

$\begin{array}{llllll}25 & 125 & 25 & 10.00 & -100.00 & 1 . e 03\end{array}$

$\begin{array}{llllll}21 & 125 & 25 & 10.00 & -100.00 & 1.003\end{array}$

$\begin{array}{llllll}22 & 125 & 25 & 10.00 & -100.00 & 1 . e 03\end{array}$

$\begin{array}{llllll}23 & 125 & 25 & 10.00 & -100.00 & 1 . e 03\end{array}$

$\begin{array}{llllll}24 & 125 & 25 & 10.00 & -100.00 & 1.003\end{array}$

$\begin{array}{llllll}0.005 & 3.00 & 1000 & 1000 & 1989 & 04\end{array}$

0 . 0.0 . 0

$\operatorname{ctr} 1$

$40 \quad 1 . e-04 \quad 08$

$\begin{array}{llll}1 & 125 & 1 & 1\end{array}$

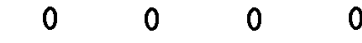

$\begin{array}{lll}1.0 & 0.0 & 1.0\end{array}$

$\begin{array}{llll}10 & 1.0 & 0.00005 & 0.005\end{array}$

00

coor

125

$\begin{array}{rlll}1 & 0.00000 & 0.00000 & 0.00000 \\ 2 & 0.12500 & 0.00000 & 0.00000 \\ 3 & 0.25000 & 0.00000 & 0.00000 \\ 4 & 0.37500 & 0.00000 & 0.00000 \\ 5 & 0.50000 & 0.00000 & 0.00000 \\ 6 & 0.00000 & 0.12500 & 0.00000 \\ 7 & 0.12500 & 0.12500 & 0.00000 \\ 8 & 0.25000 & 0.12500 & 0.00000 \\ 9 & 0.37500 & 0.12500 & 0.00000 \\ 10 & 0.50000 & 0.12500 & 0.00000 \\ 11 & 0.00000 & 0.25000 & 0.00000 \\ 12 & 0.12500 & 0.25000 & 0.00000 \\ 13 & 0.25000 & 0.25000 & 0.00000\end{array}$

Fig. 11. Input file for FEHMN for 3-D heat conduction example. 


\begin{tabular}{|c|c|c|c|}
\hline 14 & 0.37500 & 0.25000 & 0.00000 \\
\hline 15 & 0.50000 & 0.25000 & 0.00000 \\
\hline 16 & 0.00000 & 0.37500 & 0.00000 \\
\hline 17 & 0.12500 & 0.37500 & 0.00000 \\
\hline 18 & 0.25000 & 0.37500 & 0.00000 \\
\hline 19 & 0.37500 & 0.37500 & 0.00000 \\
\hline 20 & 0.50000 & 0.37500 & 0.00000 \\
\hline 21 & 0.00000 & 0.50000 & 0.00000 \\
\hline 22 & 0.12500 & 0.50000 & 0.00000 \\
\hline 23 & 0.25000 & 0.50000 & 0.00000 \\
\hline 24 & 0.37500 & 0.50000 & 0.00000 \\
\hline 25 & 0.50000 & 0.50000 & 0.00000 \\
\hline 26 & 0.00000 & 0.00000 & 0.12500 \\
\hline 27 & 0.12500 & 0.00000 & 0.12500 \\
\hline 28 & 0.25000 & 0.00000 & 0.12500 \\
\hline 29 & 0.37500 & 0.00000 & 0.12500 \\
\hline 30 & 0.50000 & 0.00000 & 0.12500 \\
\hline 31 & 0.00000 & 0.12500 & 0.12500 \\
\hline 32 & 0.12500 & 0.12500 & 0.12500 \\
\hline 33 & 0.25000 & 0.12500 & 0.12500 \\
\hline 34 & 0.37500 & 0.12500 & 0.12500 \\
\hline 35 & 0.50000 & 0.12500 & 0.12500 \\
\hline 36 & 0.00000 & 0.25000 & 0.12500 \\
\hline 37 & 0.12500 & 0.25000 & 0.12500 \\
\hline 38 & 0.25000 & 0.25000 & 0.12500 \\
\hline 39 & 0.37500 & 0.25000 & 0.12500 \\
\hline 40 & 0.50000 & 0.25000 & 0.12500 \\
\hline 41 & 0.00000 & 0.37500 & 0.12500 \\
\hline 42 & 0.12500 & 0.37500 & 0.12500 \\
\hline 43 & 0.25000 & 0.37500 & 0.12500 \\
\hline 44 & 0.37500 & 0.37500 & 0.12500 \\
\hline 45 & 0.50000 & 0.37500 & 0.12500 \\
\hline 46 & 0.00000 & 0.50000 & 0.12500 \\
\hline 47 & 0.12500 & 0.50000 & 0.12500 \\
\hline 48 & 0.25000 & 0.50000 & 0.12500 \\
\hline 49 & 0.37500 & 0.50000 & 0.12500 \\
\hline 50 & 0.50000 & 0.50000 & 0.12500 \\
\hline 51 & 0.00000 & 0.00000 & 0.25000 \\
\hline 52 & 0.12500 & 0.00000 & 0.25000 \\
\hline 53 & 0.25000 & 0.00000 & 0.25000 \\
\hline 54 & 0.37500 & 0.00000 & 0.25000 \\
\hline 55 & 0.50000 & 0.00000 & 0.25000 \\
\hline 56 & 0.00000 & 0.12500 & 0.25000 \\
\hline 57 & 0.12500 & 0.12500 & 0.25000 \\
\hline 58 & 0.25000 & 0.12500 & 0.25000 \\
\hline 59 & 0.37500 & 0.12500 & 0.25000 \\
\hline 60 & 0.50000 & 0.12500 & 0.25000 \\
\hline 61 & 0.00000 & 0.25000 & 0.25000 \\
\hline 62 & 0.12500 & 0.25000 & 0.25000 \\
\hline 63 & 0.25000 & 0.25000 & 0.25000 \\
\hline 64 & 0.37500 & 0.25000 & 0.25000 \\
\hline 65 & 0.50000 & 0.25000 & 0.25000 \\
\hline 66 & 0.00000 & 0.37500 & 0.25000 \\
\hline 67 & 0.12500 & 0.37500 & 0.25000 \\
\hline 68 & 0.25000 & 0.37500 & 0.25000 \\
\hline 69 & 0.37500 & 0.37500 & 0.25000 \\
\hline 70 & 0.50000 & 0.37500 & 0.25000 \\
\hline 71 & 0.00000 & 0.50000 & 0.25000 \\
\hline 72 & 0.12500 & 0.50000 & 0.25000 \\
\hline 73 & 0.25000 & 0.50000 & 0.25000 \\
\hline
\end{tabular}

Fig. 11. (Continued) 


\begin{tabular}{|c|c|c|c|}
\hline 74 & 0.37500 & 0.50000 & 0.25000 \\
\hline 75 & 0.50000 & 0.50000 & 0.25000 \\
\hline 76 & 0.00000 & 0.00000 & 0.37500 \\
\hline 77 & 0.12500 & 0.00000 & 0.37500 \\
\hline 78 & 0.25000 & 0.00000 & 0.37500 \\
\hline 79 & 0.37500 & 0.00000 & 0.37500 \\
\hline 80 & 0.50000 & 0.00000 & 0.37500 \\
\hline 81 & 0.00000 & 0.12500 & 0.37500 \\
\hline 82 & 0.12500 & 0.12500 & 0.37500 \\
\hline 83 & 0.25000 & 0.12500 & 0.37500 \\
\hline 84 & 0.37500 & 0.12500 & 0.37500 \\
\hline 85 & 0.50000 & 0.12500 & 0.37500 \\
\hline 86 & 0.00000 & 0.25000 & 0.37500 \\
\hline 87 & 0.12500 & 0.25000 & 0.37500 \\
\hline 88 & 0.25000 & 0.25000 & 0.37500 \\
\hline 89 & 0.37500 & 0.25000 & 0.37500 \\
\hline 90 & 0.50000 & 0.25000 & 0.37500 \\
\hline 91 & 0.00000 & 0.37500 & 0.37500 \\
\hline 92 & 0.12500 & 0.37500 & 0.37500 \\
\hline 93 & 0.25000 & 0.37500 & 0.37500 \\
\hline 94 & 0.37500 & 0.37500 & 0.37500 \\
\hline 95 & 0.50000 & 0.37500 & 0.37500 \\
\hline 96 & 0.00000 & 0.50000 & 0.37500 \\
\hline 97 & 0.12500 & 0.50000 & 0.37500 \\
\hline 98 & 0.25000 & 0.50000 & 0.37500 \\
\hline 99 & 0.37500 & 0.50000 & 0.37500 \\
\hline 100 & 0.50000 & 0.50000 & 0.37500 \\
\hline 101 & 0.00000 & 0.00000 & 0.50000 \\
\hline 102 & 0.12500 & 0.00000 & 0.50000 \\
\hline 103 & 0.25000 & 0.00000 & 0.50000 \\
\hline 104 & 0.37500 & 0.00000 & 0.50000 \\
\hline 105 & 0.50000 & 0.00000 & 0.50000 \\
\hline 106 & 0.00000 & 0.12500 & 0.50000 \\
\hline 107 & 0.12500 & 0.12500 & 0.50000 \\
\hline 108 & 0.25000 & 0.12500 & 0.50000 \\
\hline 109 & C. 37500 & 0.12500 & 0.50000 \\
\hline 110 & 0.50000 & 0.12500 & 0.50000 \\
\hline 111 & 0.00000 & 0.25000 & 0.50000 \\
\hline 112 & 0.12500 & 0.25000 & 0.50000 \\
\hline 113 & 0.25000 & 0.25000 & 0.50000 \\
\hline 114 & 0.37500 & 0.25000 & 0.50000 \\
\hline 115 & 0.50000 & 0.25000 & 0.50000 \\
\hline 116 & 0.00000 & 0.37500 & 0.50000 \\
\hline 117 & 0.12500 & 0.37500 & 0.50000 \\
\hline 118 & 0.25000 & 0.37500 & 0.50000 \\
\hline 119 & 0.37500 & 0.37500 & 0.50000 \\
\hline 120 & 0.50000 & 0.37500 & 0.50000 \\
\hline 121 & 0.00000 & 0.50000 & 0.50000 \\
\hline 122 & 0.12500 & 0.50000 & 0.50000 \\
\hline 123 & 0.25000 & 0.50000 & 0.50000 \\
\hline 124 & 0.37500 & 0.50000 & 0.50000 \\
\hline 125 & 0.50000 & 0.50000 & 0.50000 \\
\hline 0 & 0.00000 & 0.00000 & 0.00000 \\
\hline
\end{tabular}

$\begin{array}{rrrrrrrrr}\text { elem } & & & & & & & & \\ \text { 8, } & 64, & 0 & & & & & & \\ 1 & 1 & 2 & 7 & 6 & 26 & 27 & 32 & 31 \\ 2 & 2 & 3 & 8 & 7 & 27 & 28 & 33 & 32 \\ 3 & 3 & 4 & 9 & 8 & 28 & 29 & 34 & 33 \\ 4 & 4 & 5 & 10 & 9 & 29 & 30 & 35 & 34 \\ 5 & 6 & 7 & 12 & 11 & 31 & 32 & 37 & 36\end{array}$

Fig. 11. Continued 


\begin{tabular}{|c|c|c|c|c|c|c|c|c|}
\hline 6 & 7 & 8 & 13 & 12 & 32 & 33 & 38 & 37 \\
\hline 7 & 8 & 9 & 14 & 13 & 33 & 34 & 39 & 38 \\
\hline 8 & 9 & 10 & 15 & 14 & 34 & 35 & 40 & 39 \\
\hline 9 & 11 & 12 & 17 & 16 & 36 & 37 & 42 & 41 \\
\hline 10 & 12 & 13 & 18 & 17 & 37 & 38 & 43 & 42 \\
\hline 11 & 13 & 14 & 19 & 18 & 38 & 39 & 44 & 43 \\
\hline 12 & 14 & 15 & 20 & 19 & 39 & 40 & 45 & 44 \\
\hline 13 & 16 & 17 & 22 & 21 & 41 & 42 & 47 & 46 \\
\hline 14 & 17 & 18 & 23 & 22 & 42 & 43 & 48 & 47 \\
\hline 15 & 18 & 19 & 24 & 23 & 43 & 44 & 49 & 48 \\
\hline 16 & 19 & 20 & 25 & 24 & 44 & 45 & 50 & 49 \\
\hline 17 & 26 & 27 & 32 & 31 & 51 & 52 & 57 & 56 \\
\hline 18 & 27 & 28 & 33 & 32 & 52 & 53 & 58 & 57 \\
\hline 19 & 28 & 29 & 34 & 33 & 53 & 54 & 59 & 58 \\
\hline 20 & 29 & 30 & 35 & 34 & 54 & 55 & 60 & 59 \\
\hline 21 & 31 & 32 & 37 & 36 & 56 & 57 & 62 & 61 \\
\hline 22 & 32 & 33 & 38 & 37 & 57 & 58 & 63 & 62 \\
\hline 23 & 33 & 34 & 39 & 38 & 58 & 59 & 64 & 63 \\
\hline 24 & 34 & 35 & 40 & 39 & 59 & 60 & 65 & 64 \\
\hline 25 & 36 & 37 & 42 & 41 & 61 & 62 & 67 & 66 \\
\hline 26 & 37 & 38 & 43 & 42 & 62 & 63 & 68 & 67 \\
\hline 27 & 38 & 39 & 44 & 43 & 63 & 64 & 69 & 68 \\
\hline 28 & 39 & 40 & 45 & 44 & 64 & 65 & 70 & 69 \\
\hline 29 & 41 & 42 & 47 & 46 & 66 & 67 & 72 & 71 \\
\hline 30 & 42 & 43 & 48 & 47 & 67 & 68 & 73 & 72 \\
\hline 31 & 43 & 44 & 49 & 48 & 68 & 69 & 74 & 73 \\
\hline 32 & 44 & 45 & 50 & 49 & 69 & 70 & 75 & 74 \\
\hline 33 & 51 & 52 & 57 & 56 & 76 & 77 & 82 & 81 \\
\hline 34 & 52 & 53 & 58 & 57 & 77 & 78 & 83 & 82 \\
\hline 35 & 53 & 54 & 59 & 58 & 78 & 79 & 84 & 83 \\
\hline 36 & 54 & 5.5 & 60 & 59 & 79 & 80 & 85 & 84 \\
\hline 37 & 56 & 57 & 62 & 61 & 81 & 82 & 87 & 86 \\
\hline 38 & 57 & 58 & 63 & 62 & 82 & 83 & 88 & 87 \\
\hline 39 & 58 & 59 & 64 & 63 & 83 & 84 & 89 & 88 \\
\hline 40 & 59 & 60 & 65 & 64 & 84 & 85 & 90 & 89 \\
\hline 41 & 61 & 62 & 67 & 66 & 86 & 87 & 92 & 91 \\
\hline 42 & 62 & 63 & 68 & 67 & 87 & 88 & 93 & 92 \\
\hline 43 & 63 & 64 & 69 & 68 & 88 & 89 & 94 & 93 \\
\hline 44 & 64 & 65 & 70 & 69 & 89 & 90 & 95 & 94 \\
\hline 45 & 66 & 67 & 72 & 71 & 91 & 92 & 97 & 96 \\
\hline 46 & 67 & 68 & 73 & 72 & 92 & 93 & 98 & 97 \\
\hline 47 & 68 & 69 & 74 & 73 & 93 & 94 & 99 & 98 \\
\hline 48 & 69 & 70 & 75 & 74 & 94 & 95 & 100 & 99 \\
\hline 49 & 76 & 77 & 82 & 81 & 101 & 102 & 107 & 106 \\
\hline 50 & 77 & 78 & 83 & 82 & 102 & 103 & 108 & 107 \\
\hline 51 & 78 & 79 & 84 & 83 & 103 & 104 & 109 & 108 \\
\hline 52 & 79 & 80 & 85 & 84 & 104 & 105 & 110 & 109 \\
\hline 53 & 81 & 82 & 87 & 86 & 106 & 107 & 112 & 111 \\
\hline 54 & 82 & 83 & 88 & 87 & 107 & 108 & 113 & 112 \\
\hline 55 & 83 & 84 & 89 & 88 & 108 & 109 & 114 & 113 \\
\hline 56 & 84 & 85 & 90 & 89 & 109 & 110 & 115 & 114 \\
\hline 57 & 86 & 87 & 92 & 91 & 111 & 112 & 117 & 116 \\
\hline 58 & 87 & 88 & 93 & 92 & 11.2 & 113 & 118 & 117 \\
\hline 59 & 88 & 89 & 94 & 93 & 113 & 114 & 119 & 118 \\
\hline 60 & 89 & 90 & 95 & 94 & 114 & 115 & 120 & 119 \\
\hline 61 & 91 & 92 & 97 & 96 & 116 & 117 & 122 & 121 \\
\hline 62 & 92 & 93 & 98 & 97 & 117 & 118 & 123 & 122 \\
\hline 63 & 93 & 94 & 99 & 98 & 118 & 119 & 124 & 123 \\
\hline 64 & 94 & 95 & 100 & 99 & 119 & 120 & 125 & 124 \\
\hline 0 & 0 & 0 & 0 & 0 & 0 & 0 & 0 & 0 \\
\hline
\end{tabular}

Fig. 11. Continued 


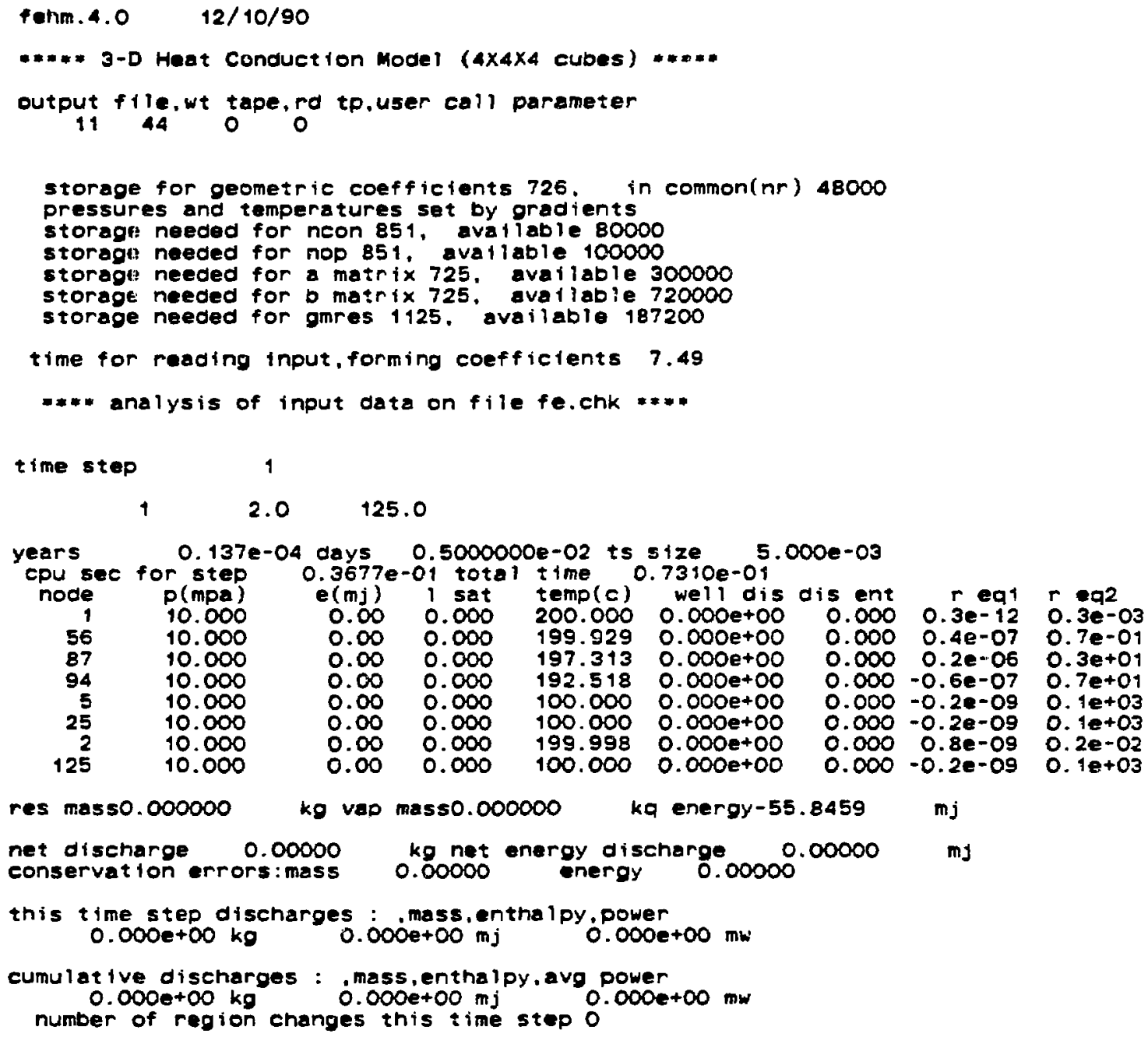

Fig. 12. Computer output for the 3-D heat conduction example. 


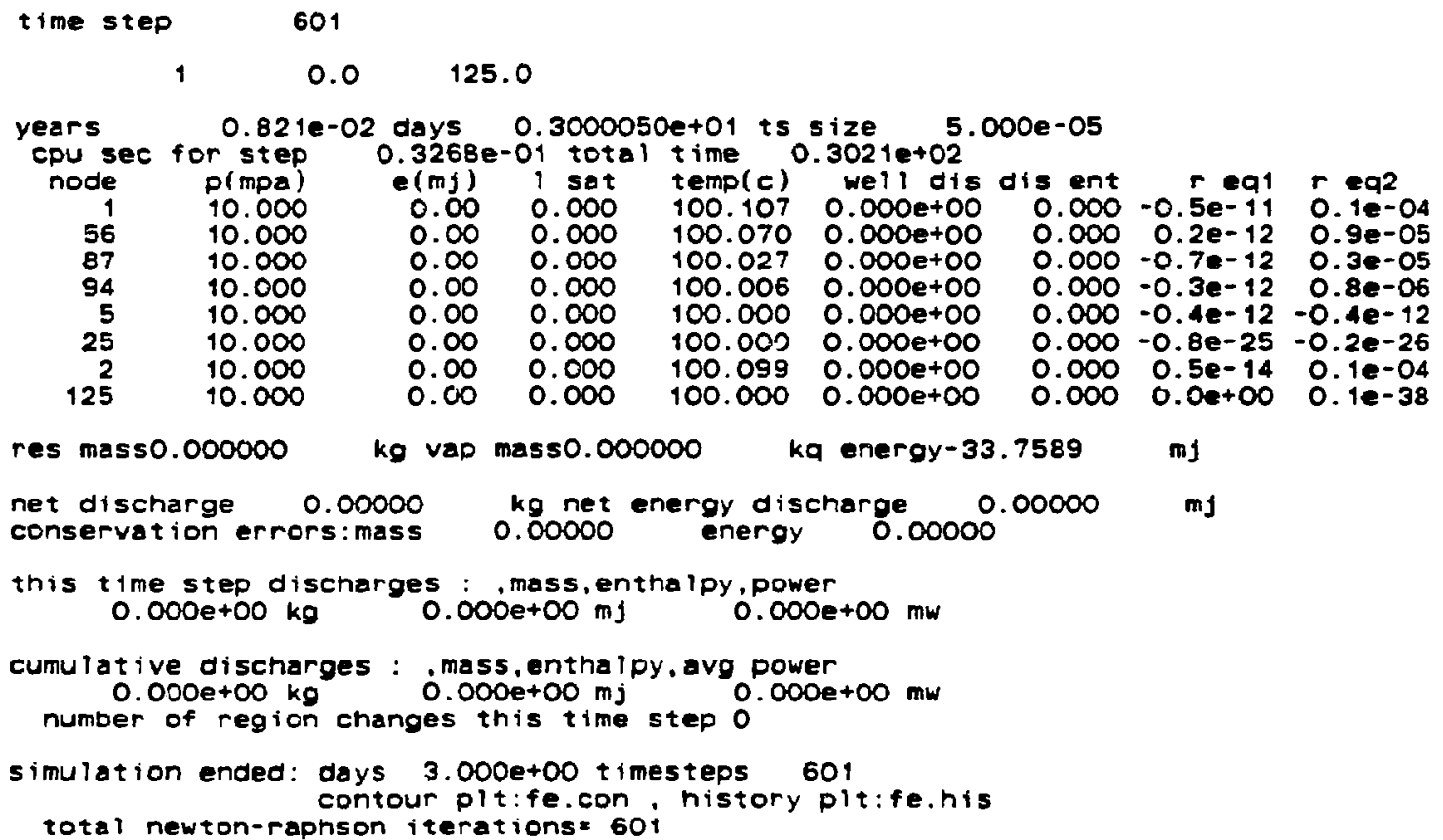

Fig. 12. Continued 


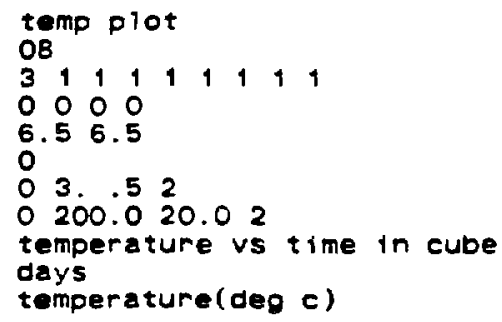

Fig. 13. Instruction file (HIS.INS) for postprocessor FEHPLTR for the heat conduction problem. 
fehm.4.0 12/10/90 temperature vs time in cube

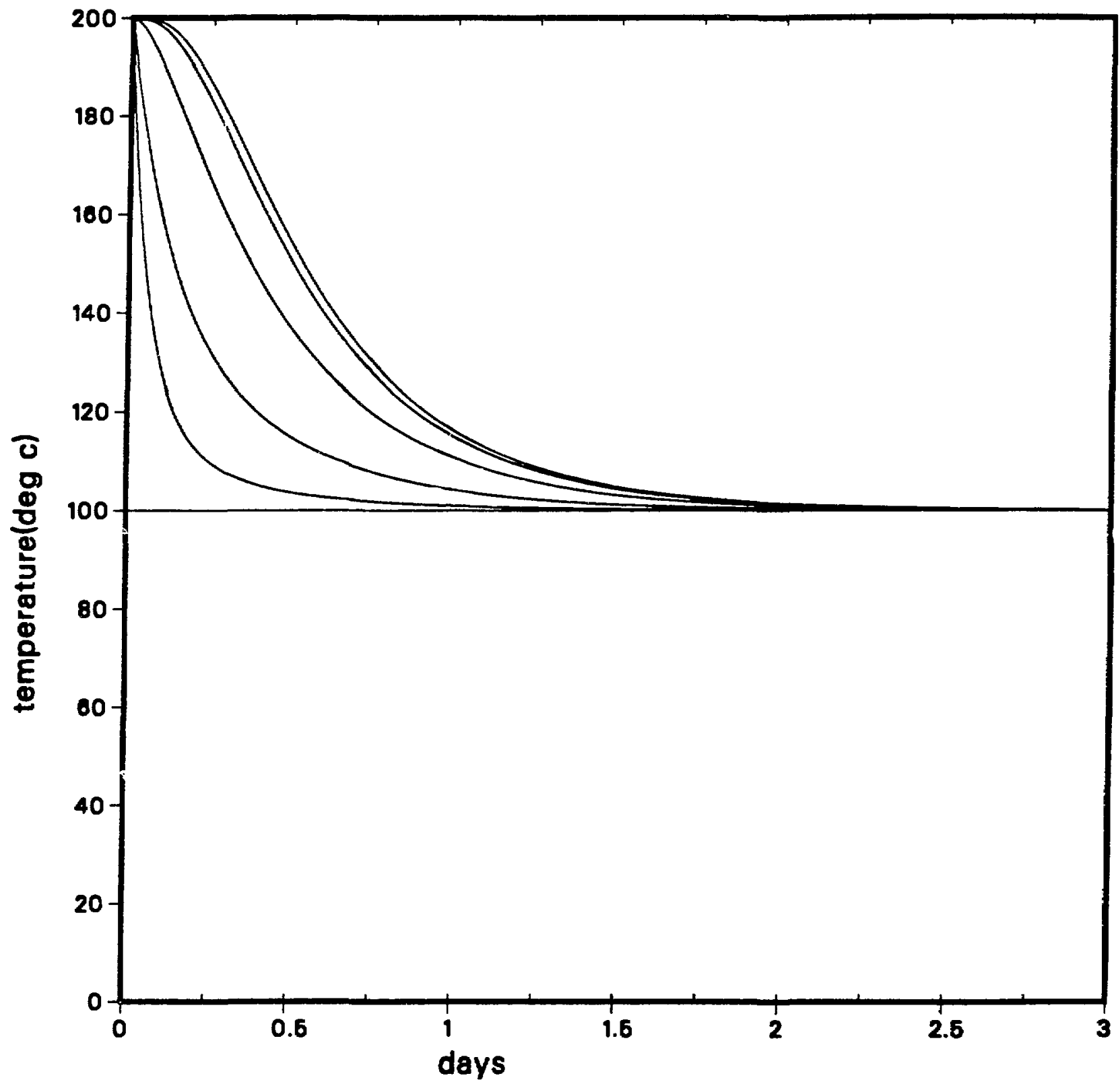

Fig. 14. History plot for the heat conduction problem. 


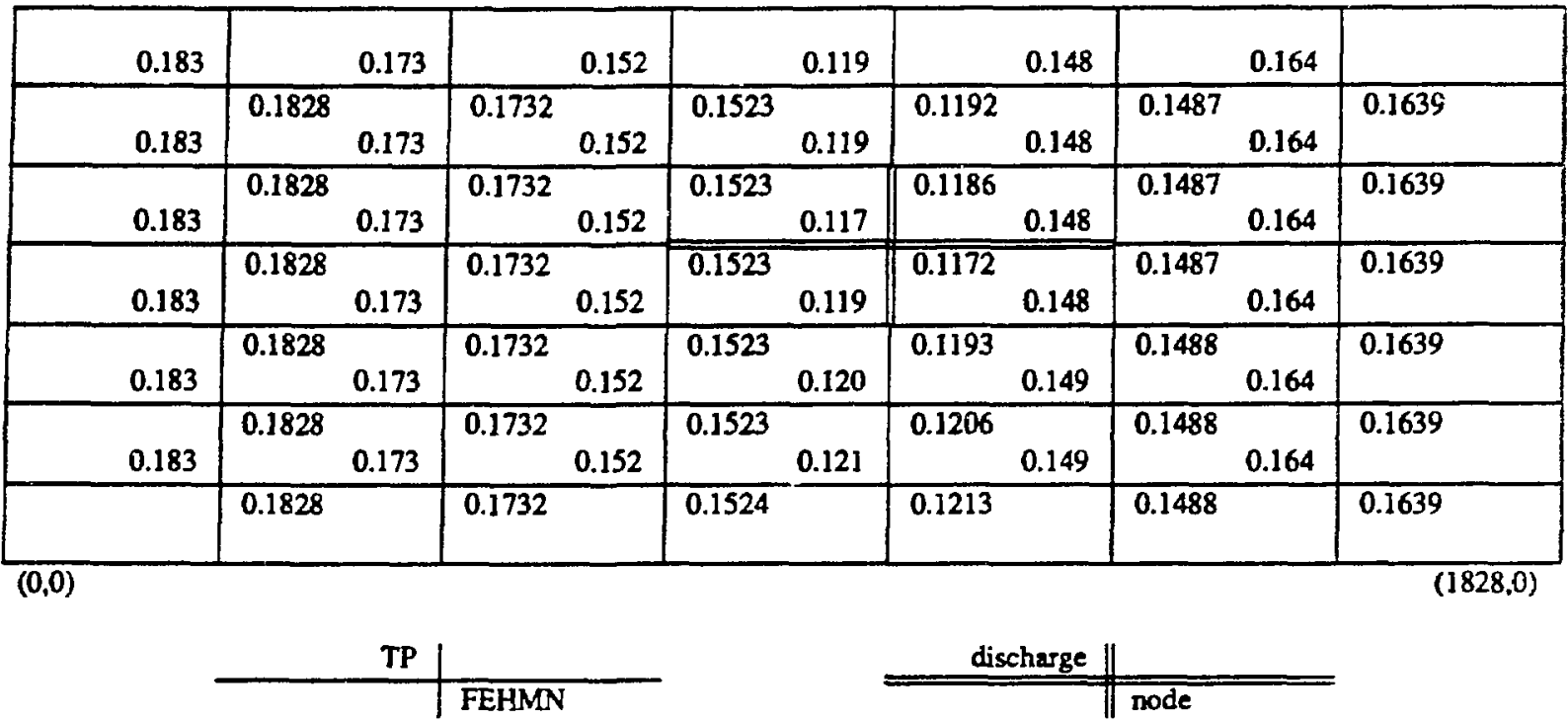

Fig. 15. Solution domain and results for the Toronyi example.

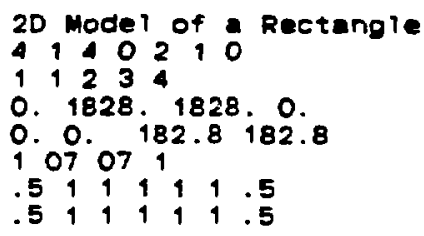

Fig. 16. Input for GENMSH for the Toronyi example. 


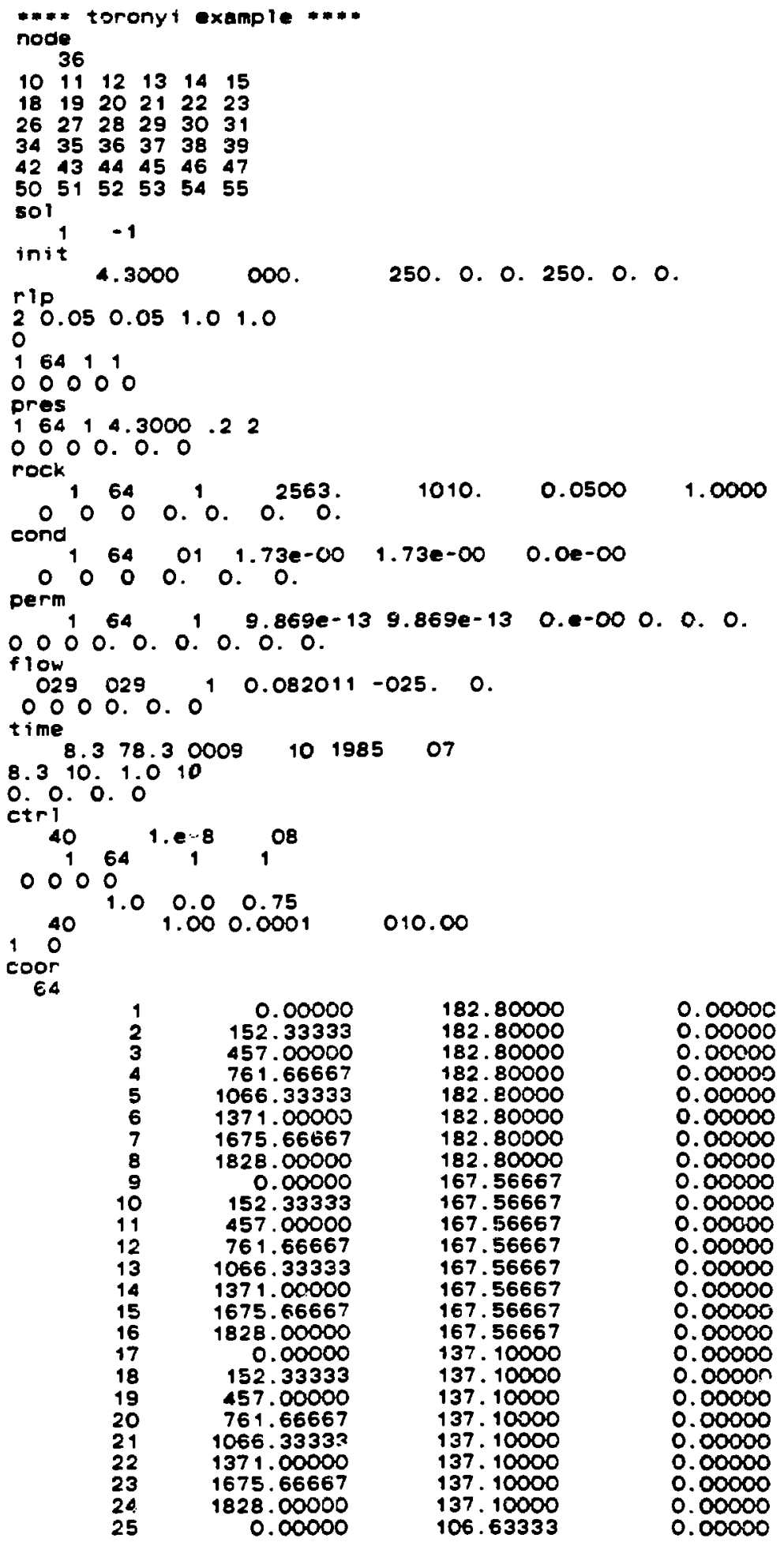

Fig. 17. Input file for FEHMN for Toronyi example. 


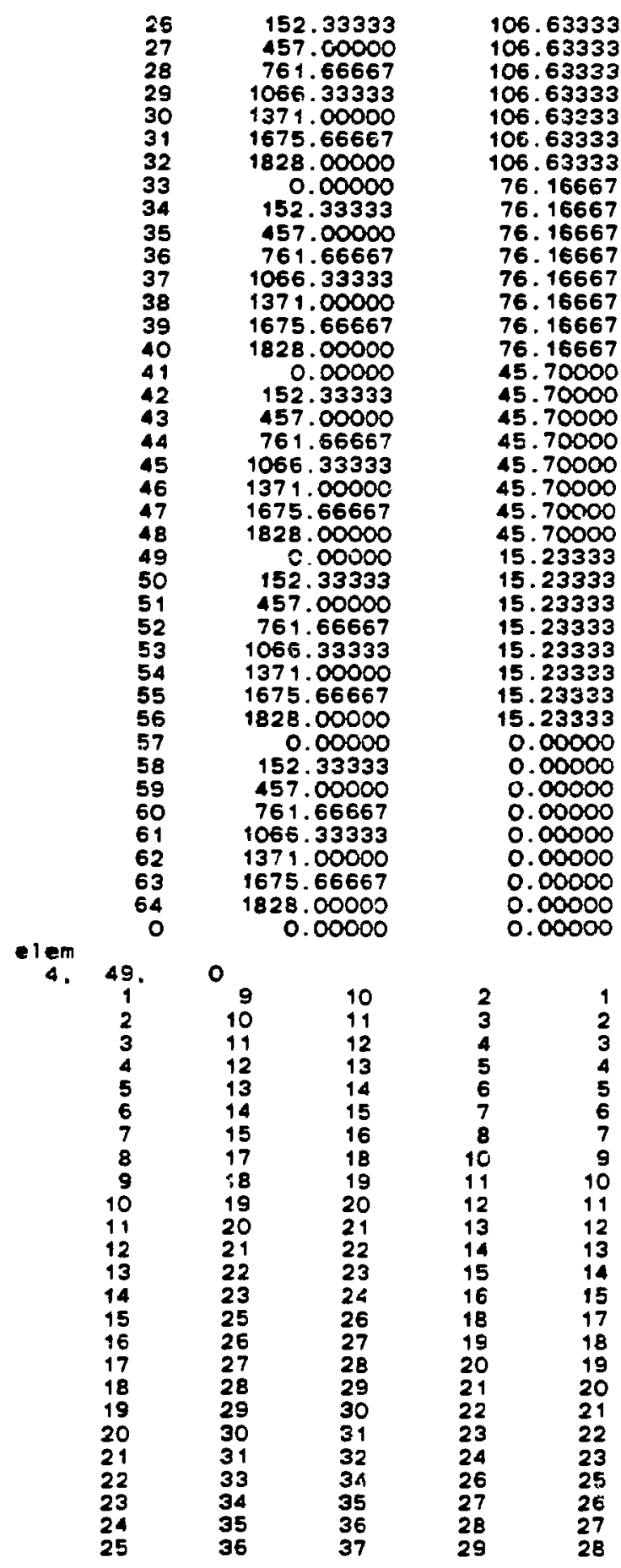

Fig. 17. (Continued) 


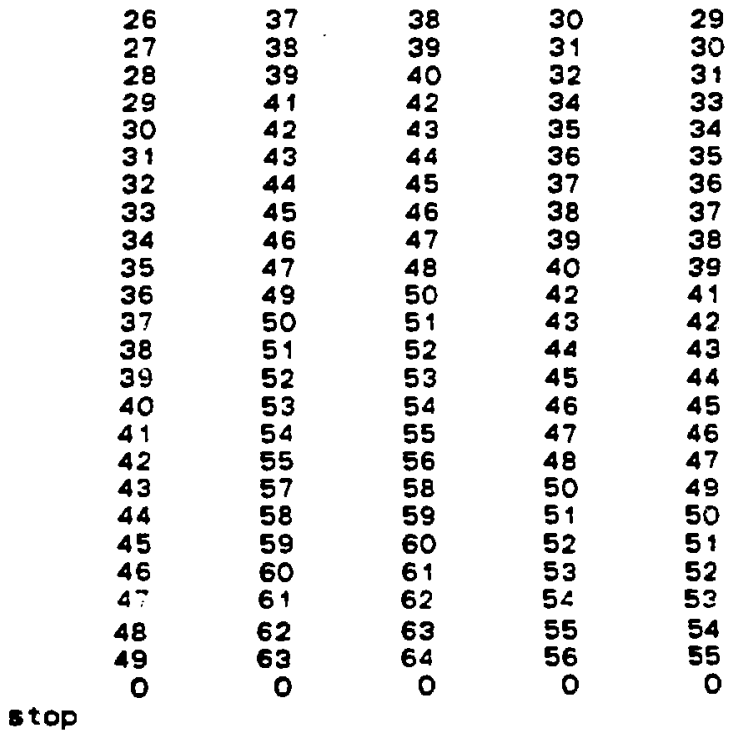

Fig. 17. Continued. 


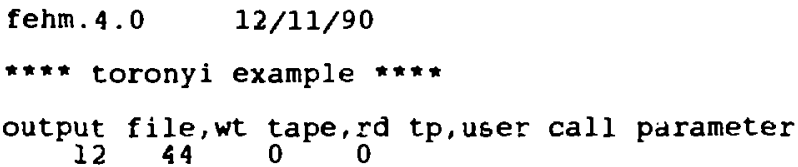

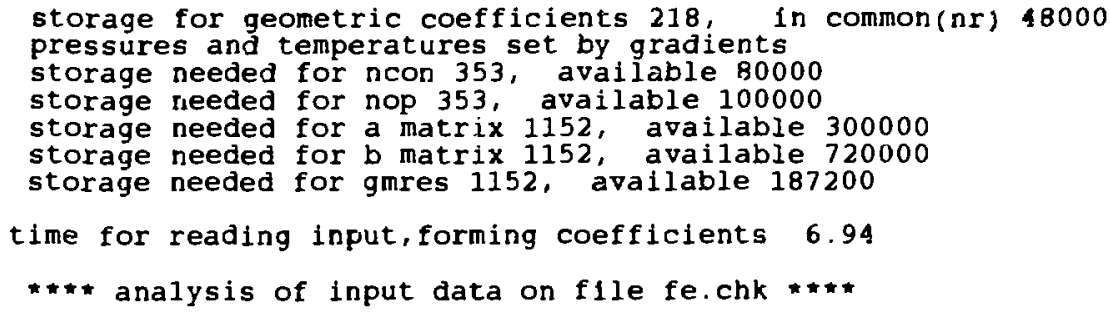

\begin{tabular}{|c|c|c|c|c|c|c|c|c|}
\hline $\begin{array}{c}\text { ears } \\
\text { cpu sec } \\
\text { node } \\
10 \\
11 \\
12 \\
13 \\
14 \\
15 \\
18 \\
19 \\
20 \\
21 \\
22 \\
23 \\
26 \\
27 \\
28 \\
29 \\
30 \\
31 \\
34 \\
35 \\
36 \\
37 \\
38 \\
39 \\
42 \\
43 \\
44 \\
45 \\
46 \\
47 \\
50 \\
51 \\
52 \\
53 \\
54 \\
55\end{array}$ & $\begin{array}{r}0.227 \epsilon \\
\text { or step } \\
\text { p(mpa) } \\
4.300 \\
4.299 \\
4.293 \\
4.265 \\
4.293 \\
4.298 \\
4.300 \\
4.299 \\
4.293 \\
4.264 \\
4.293 \\
4.298 \\
4.300 \\
4.299 \\
4.293 \\
4.261 \\
4.293 \\
4.298 \\
4.300 \\
4.299 \\
4.293 \\
4.265 \\
4.293 \\
4.298 \\
4.300 \\
4.299 \\
4.293 \\
4.268 \\
4.293 \\
4.298 \\
4.300 \\
4.299 \\
4.293 \\
4.269 \\
4.293 \\
4.298\end{array}$ & $\begin{array}{c}\text { days } \\
0.1750 \mathrm{e} \\
\text { e(mj) } \\
1.27 \\
1.27 \\
1.28 \\
1.29 \\
1.28 \\
1.27 \\
1.27 \\
1.27 \\
1.28 \\
1.29 \\
1.28 \\
1.27 \\
1.27 \\
1.27 \\
1.28 \\
1.29 \\
1.28 \\
1.27 \\
1.27 \\
1.27 \\
1.28 \\
1.29 \\
1.28 \\
1.27 \\
1.27 \\
1.27 \\
1.28 \\
1.29 \\
1.28 \\
1.27 \\
1.27 \\
1.27 \\
1.28 \\
1.29 \\
11.28 \\
1.27\end{array}$ & $\begin{array}{c}0.830000 \\
+00 \text { total } \\
1 \text { sat } \\
0.200 \\
0.199 \\
0.196 \\
0.182 \\
0.196 \\
0.199 \\
0.200 \\
0.199 \\
0.196 \\
0.181 \\
0.196 \\
0.199 \\
0.200 \\
0.199 \\
0.196 \\
0.179 \\
0.196 \\
0.199 \\
0.200 \\
0.199 \\
0.196 \\
0.182 \\
0.196 \\
0.199 \\
0.200 \\
0.199 \\
0.196 \\
0.183 \\
0.196 \\
0.199 \\
0.200 \\
0.199 \\
0.196 \\
0.124 \\
0.196 \\
0.199\end{array}$ & $\begin{array}{l}\text { loetol ts } \\
t \text { ime } \\
\text { temp (c) } \\
254.600 \\
254.585 \\
254.507 \\
254.123 \\
254.506 \\
254.580 \\
254.600 \\
254.585 \\
254.507 \\
254.101 \\
254.506 \\
254.580 \\
254.600 \\
254.585 \\
254.507 \\
254.055 \\
254.506 \\
254.580 \\
254.600 \\
254.585 \\
254.508 \\
254.118 \\
254.507 \\
254.580 \\
254.600 \\
254.585 \\
254.508 \\
254.59\end{array}$ & $\begin{array}{c}\text { size } \\
0.2091 \mathrm{e}+00 \\
\text { we1 dis } \\
0.000 \mathrm{e}+00 \\
0.000 \mathrm{e}+00 \\
0.000 \mathrm{e}+00 \\
0.000 \mathrm{e}+00 \\
0.000 \mathrm{e}+00 \\
0.000 \mathrm{e}+00 \\
0.000 \mathrm{e}+00 \\
0.000 \mathrm{e}+00 \\
0.000 \mathrm{e}+00 \\
0.000 \mathrm{e}+00 \\
0.000 \mathrm{e}+00 \\
0.000 \mathrm{e}+00 \\
0.000 \mathrm{e}+00 \\
0.000 \mathrm{e}+00 \\
0.000 \mathrm{e}+00 \\
0.820 \mathrm{e}-01 \\
0.000 \mathrm{e}+00 \\
0.000 \mathrm{e}+00 \\
0.000 \mathrm{e}+00 \\
0.000 \mathrm{e}+00 \\
0.000 \mathrm{e}+00 \\
0.000 \mathrm{e}+00 \\
0.00 \mathrm{j}+00 \\
0.00 \mathrm{ee}+00 \\
0.010 \mathrm{e}+00 \\
0.00 \mathrm{e}+00 \\
0.000 \mathrm{e}+00 \\
0.000 \mathrm{e}+00 \\
0.000 \mathrm{e}+00 \\
0.000 \mathrm{e}+00 \\
0.000 \mathrm{e}+00 \\
0.000 \mathrm{e}+00 \\
0.000 \mathrm{e}+00 \\
0.000 \mathrm{e}+00 \\
0.000 \mathrm{e}+00 \\
0.000 \mathrm{e}+00\end{array}$ & $\begin{array}{r}\text { dis ent } \\
0.000 \\
0.000 \\
0.000 \\
0.000 \\
0.000 \\
0.000 \\
0.000 \\
0.000 \\
0.000 \\
0.000 \\
0.000 \\
0.000 \\
0.000 \\
0.000 \\
0.000 \\
2.792 \\
0.000 \\
0.000 \\
0.000 \\
0.000 \\
0.000 \\
0.000 \\
0.000 \\
0.000 \\
0.000 \\
0.000 \\
0.000 \\
0.000 \\
0.000 \\
0.000 \\
0.000 \\
0.000 \\
0.000 \\
0.000 \\
0.000 \\
0.000 \\
\end{array}$ & 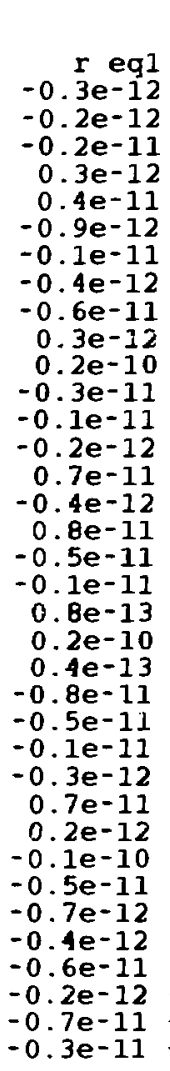 & $\begin{array}{r}-0 . \\
-0 . \\
0 . \\
0 . \\
0 . \\
-0 . \\
-0 . \\
-0 . \\
-0 . \\
0 . \\
-0 . \\
-0 . \\
-0 . \\
-0 . \\
-0 . \\
-0 . \\
-0 . \\
-0 . \\
-0 .\end{array}$ \\
\hline
\end{tabular}

res mass0.287727e+07 kg vap mass 290027 . kg energy0.212729e+09 mj

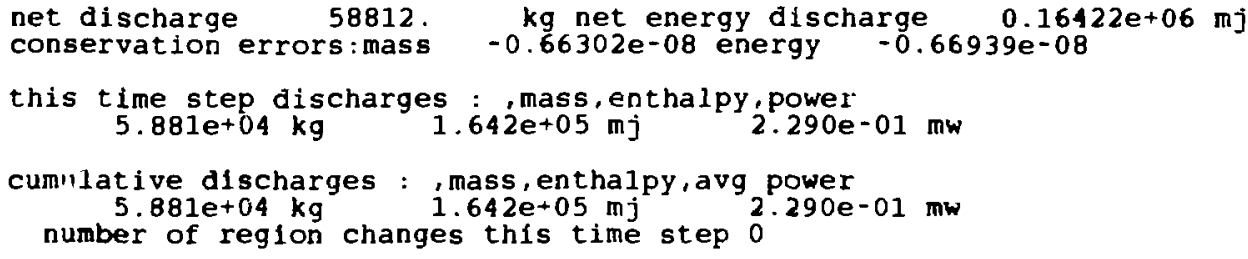

Fig. 18. Output for the Toronyi example. 


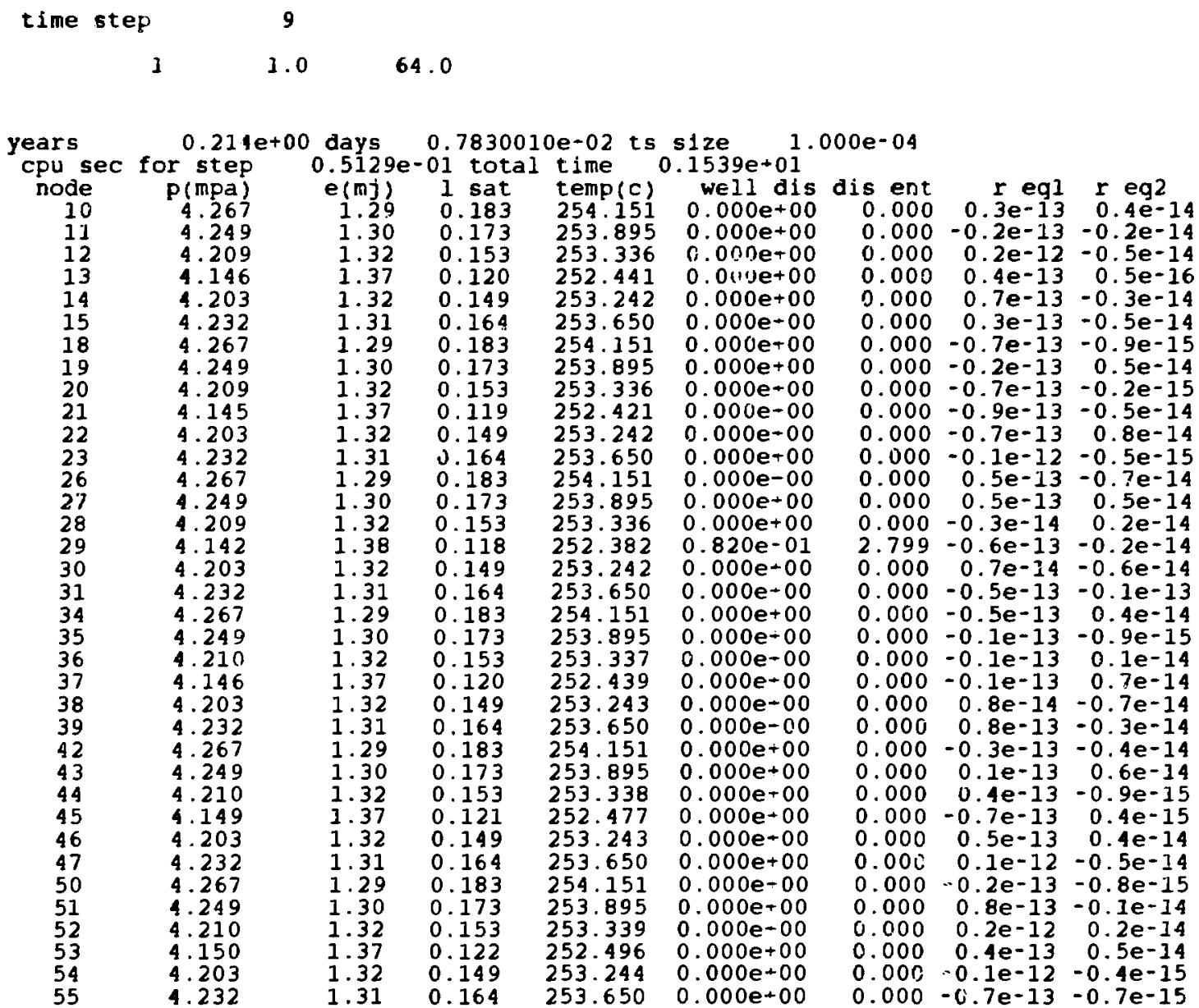

res mass0.238127e+07 kg vap mass $298427 . \quad \mathrm{kg}$ energy0.211341e+09 $\mathrm{mj}$

net discharge $0.554 \mathrm{Ble}+06 \mathrm{~kg}$ net energy discharge $0.15518 \mathrm{e}+07 \mathrm{mj}$

conservation errors:mass $-0.29266 \mathrm{e}-08$ energy $-0.43337 \mathrm{e}-08$

this time step discharges : mass, enthalpy, power $7.086 \mathrm{e}-01 \mathrm{~kg} \quad 1.983 \mathrm{e}+00 \mathrm{mj} \quad 2.295 \mathrm{e}-01 \mathrm{mw}$

cumulative discharges : , mass, enthalpy, avg power $5.548 \mathrm{e}-05 \mathrm{~kg} \quad 1.552 \mathrm{e}-06 \mathrm{mj} \quad 2.294 \mathrm{e}-01 \mathrm{mw}$ number of region changes this time step 0

simulation ended: days $7.830 e+01$ timesteps 9 total newton-raphson iterations* 19

Fig. 18. (Continued) 


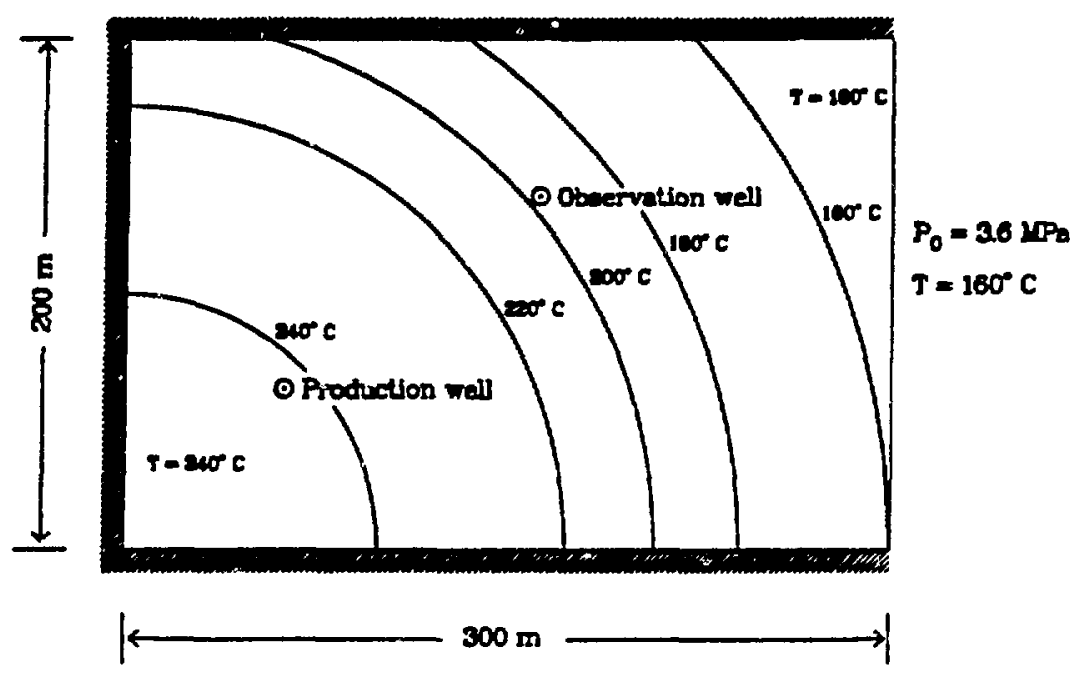

Fig. 19. Schematic diagram of the geometry and boundary conditions for the DOE code comparison project problem.

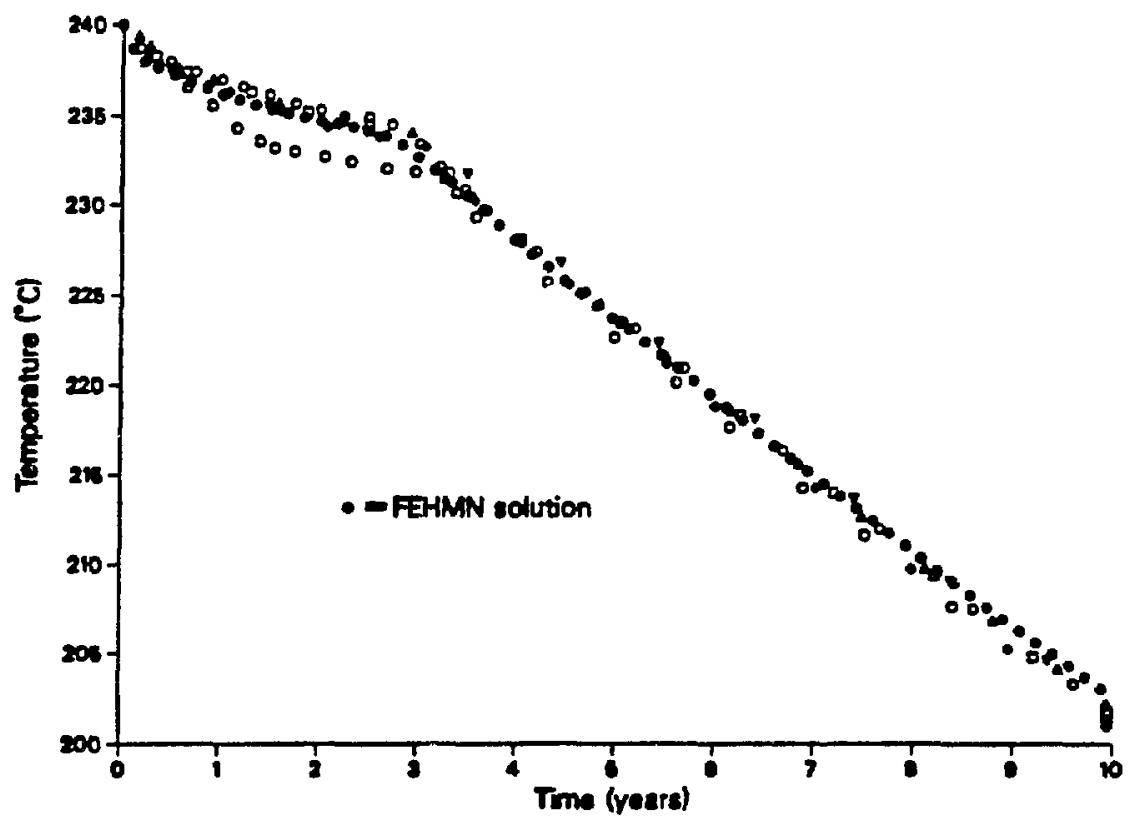

Fig. 20. Comparison of FEHMN production well temperatures with results from other codes. 


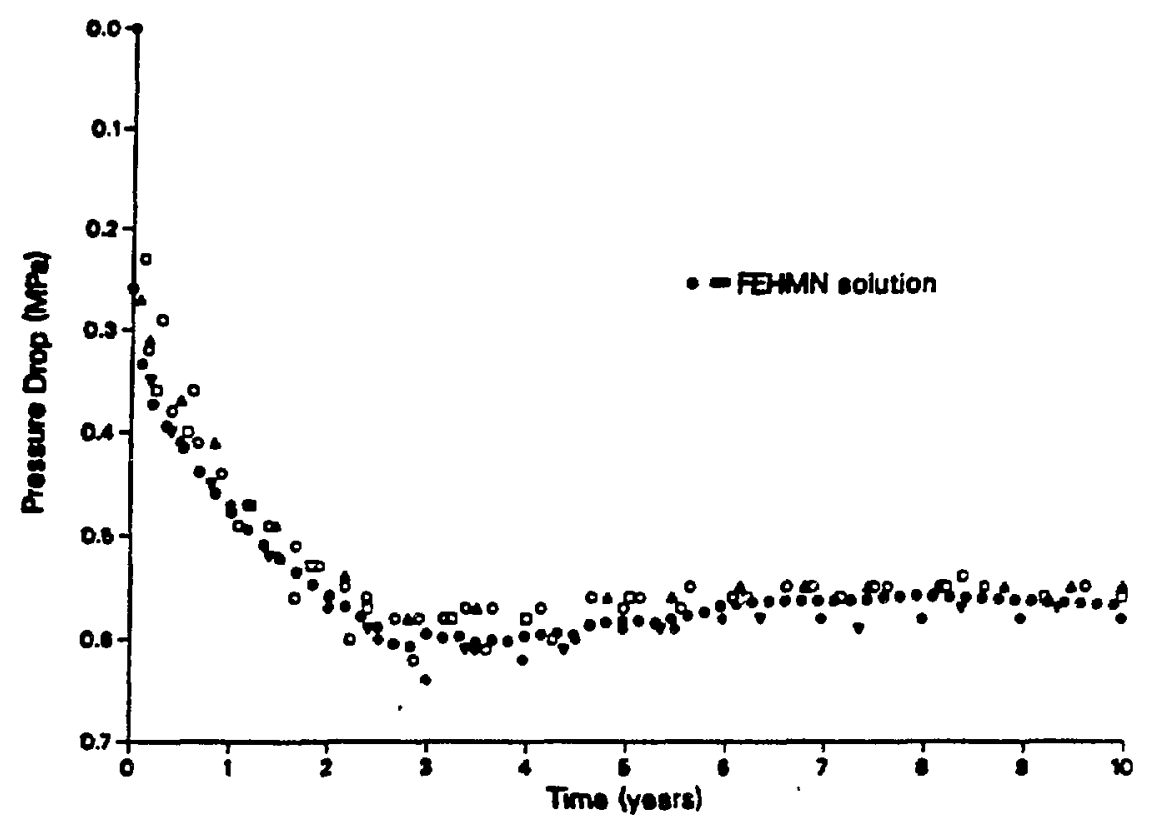

Fig. 21. Comparison of FEHMN production well pressure with results from other codes.

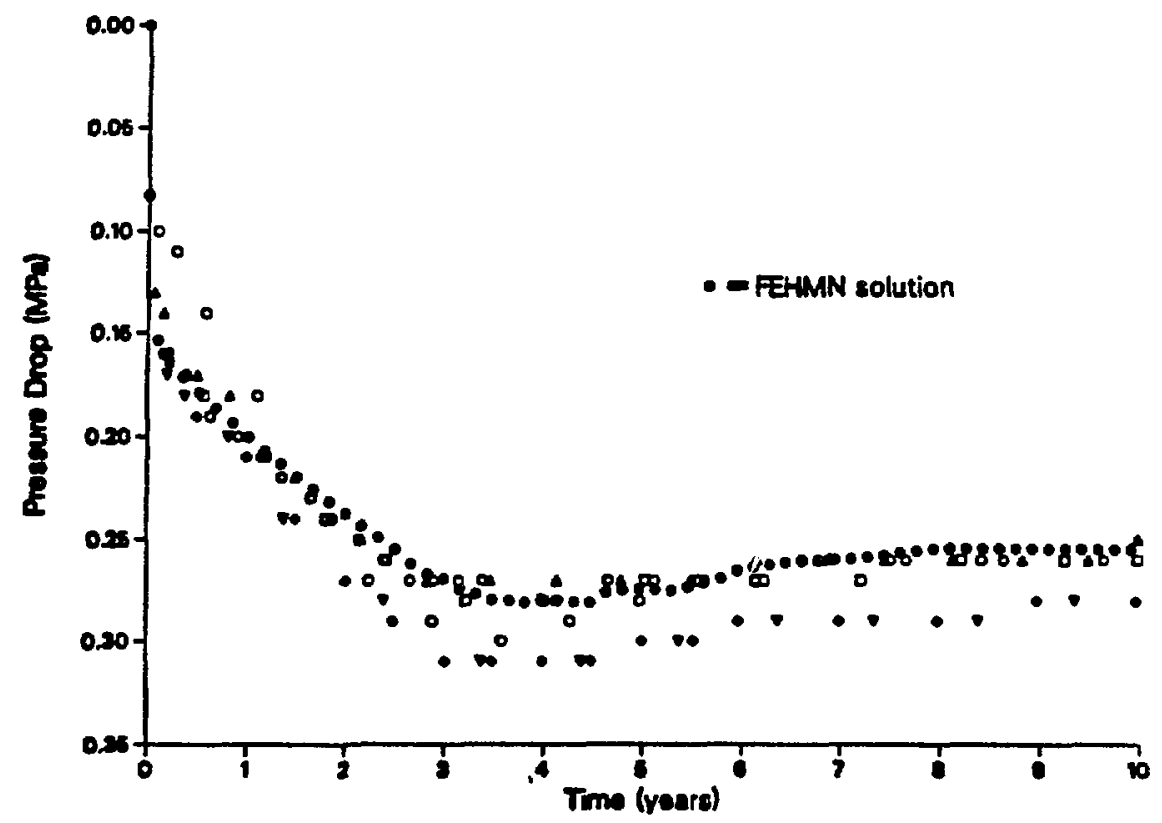

Fig. 22. Comparison of FEHMN observation well pressure with results from other codes. 
DOE Code Comparison Project, Problem 5, Case A

$\begin{array}{llllllll}8 & 1 & 8 & 0 & 2 & 1 & 0\end{array}$

$\begin{array}{lllllllll}1 & 1 & 2 & 3 & 4 & 5 & 6 & 7 & 8\end{array}$

0. 150.300.300.300.150.0.0.

0.0.0.100.200.200.200. 100.

$\begin{array}{llll}1 & 13 & 9 & 1\end{array}$

$\begin{array}{lllllllllllllll}.5 & 1 & 1 & 1 & 1 & 1 & 1 & 1 & 1 & 1 & 1 & 1 & .5\end{array}$

$\begin{array}{llllllllll}5 & 1 & 1 & 1 & 1 & 1 & 1 & 1 & .5\end{array}$

Fig. 23. Input for GENMSH for the DOE example.

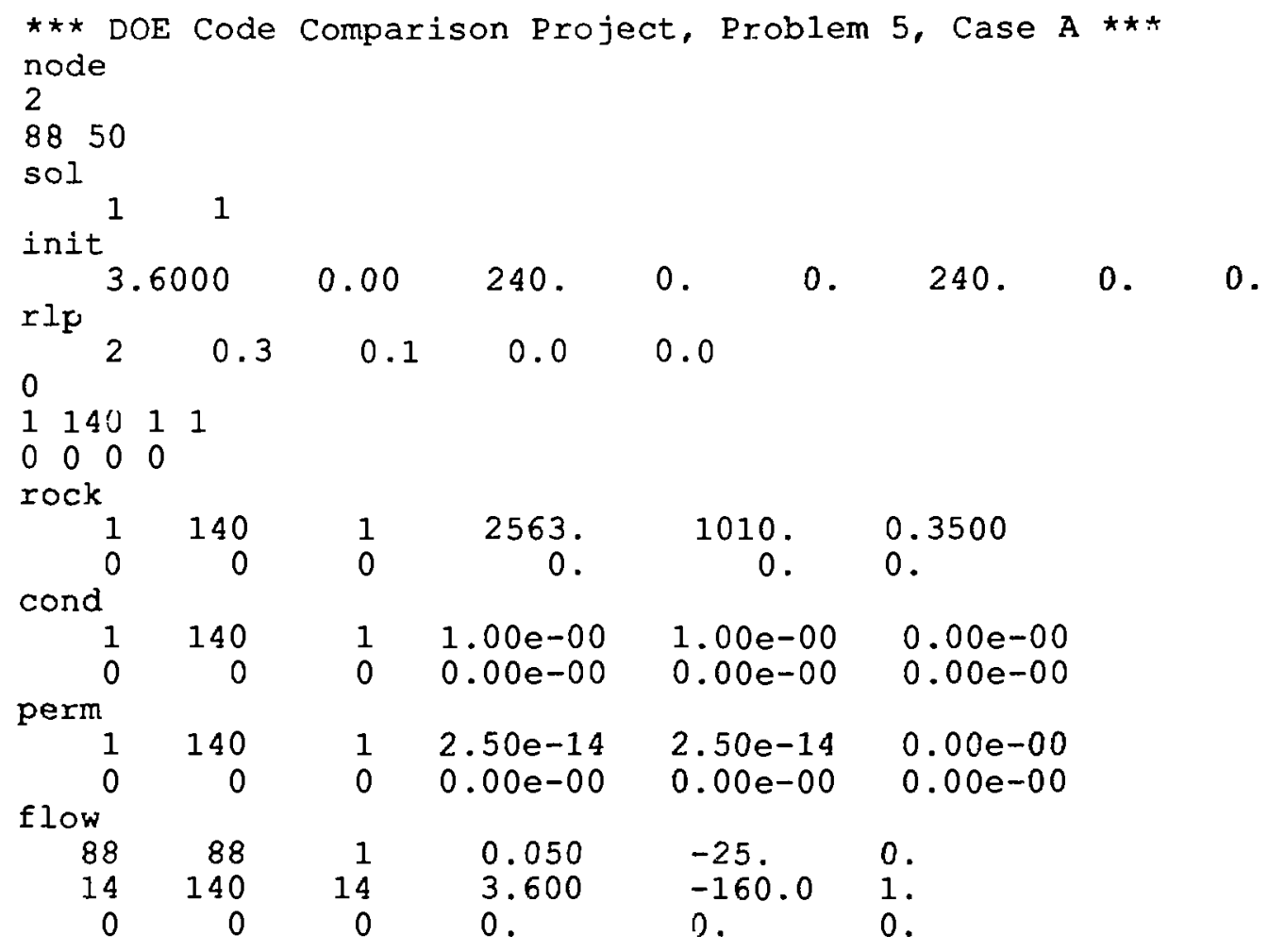

Fig. 24. Input for FEHMN for the DOE example. 


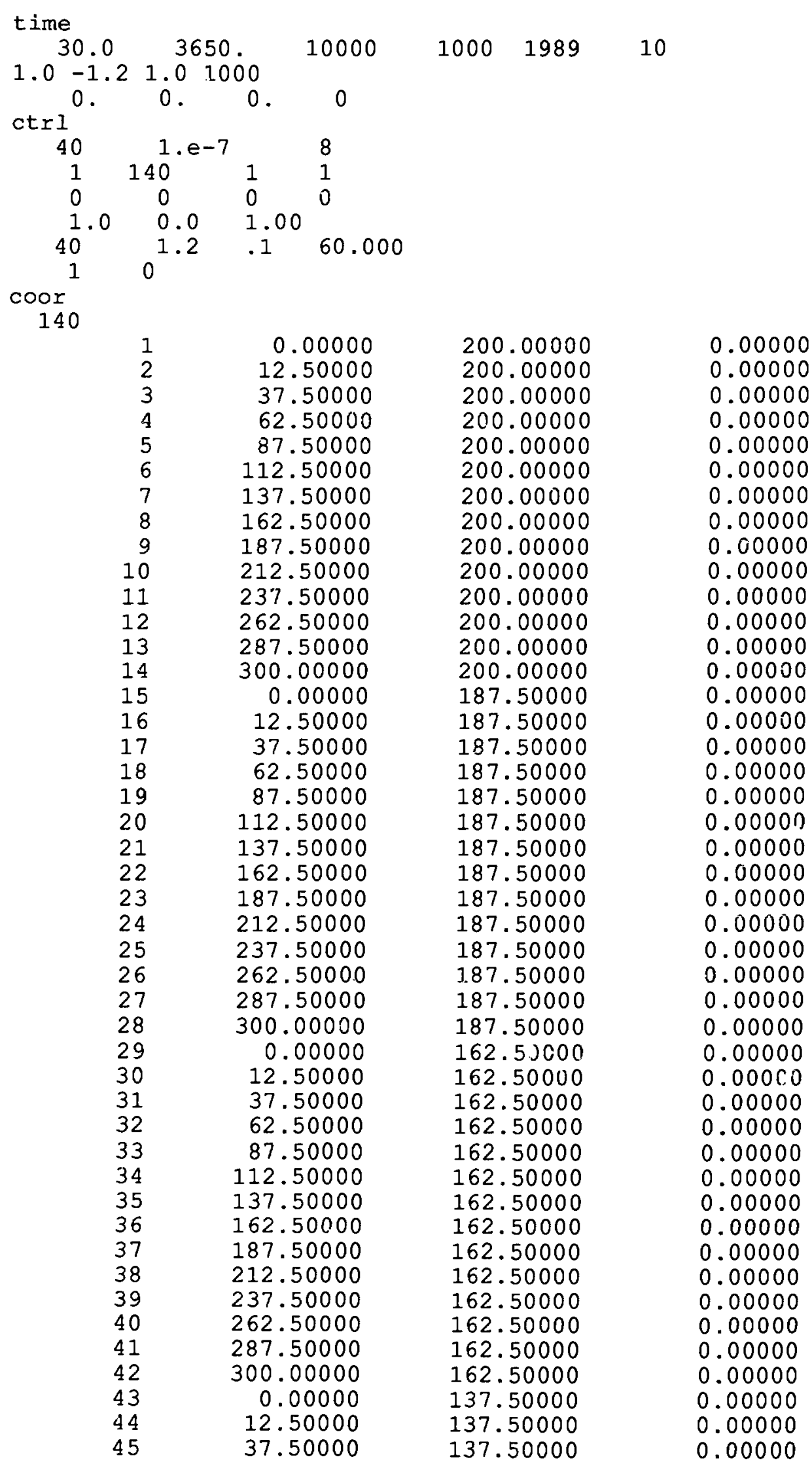

Fig. 24. (continued) 


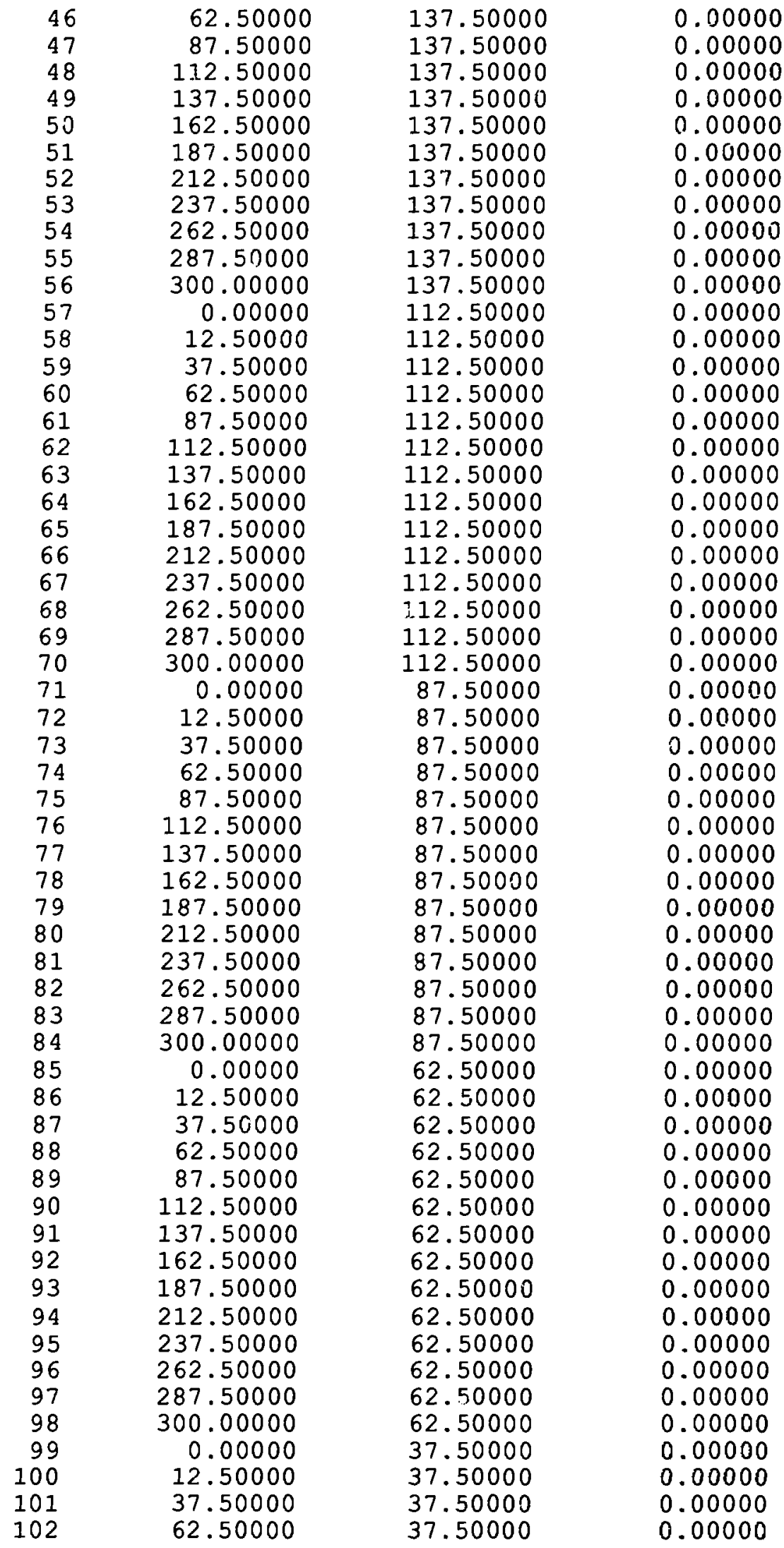

Fig. 24. (continued) 


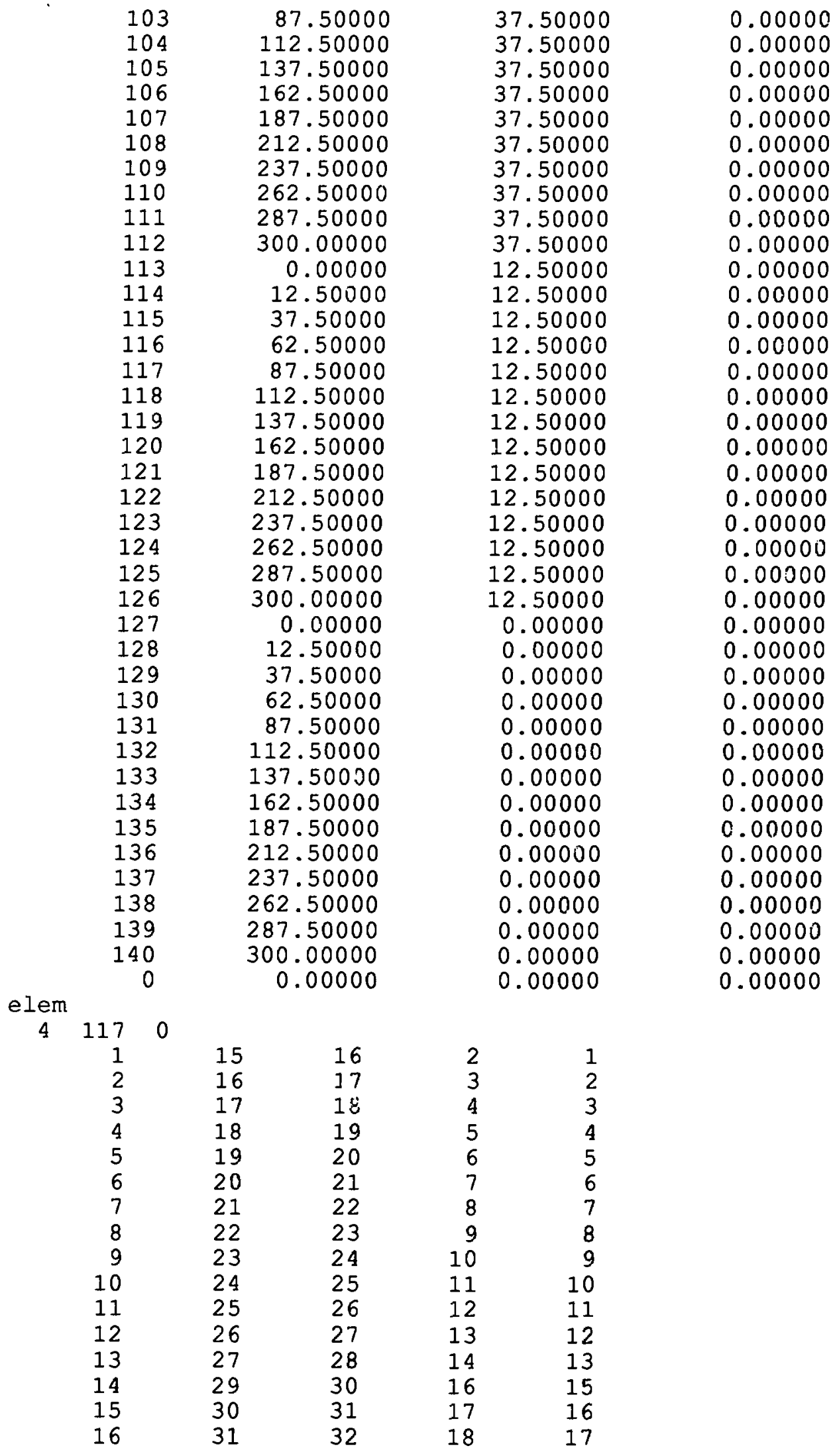

Fig. 24. (continued) 


$\begin{array}{lllll}17 & 32 & 33 & 19 & 18 \\ 18 & 33 & 34 & 20 & 19 \\ 19 & 34 & 35 & 21 & 20 \\ 20 & 35 & 36 & 22 & 21 \\ 21 & 36 & 37 & 23 & 22 \\ 22 & 37 & 38 & 24 & 23 \\ 23 & 38 & 39 & 25 & 24 \\ 24 & 39 & 40 & 26 & 25 \\ 25 & 40 & 41 & 27 & 26 \\ 26 & 41 & 42 & 28 & 27 \\ 27 & 43 & 44 & 30 & 29 \\ 28 & 44 & 45 & 31 & 30 \\ 29 & 45 & 46 & 32 & 31 \\ 30 & 46 & 47 & 33 & 32 \\ 31 & 47 & 48 & 34 & 33 \\ 32 & 48 & 49 & 35 & 34 \\ 33 & 49 & 30 & 36 & 35 \\ 34 & 50 & 51 & 37 & 36 \\ 35 & 51 & 52 & 38 & 37 \\ 36 & 52 & 53 & 39 & 38 \\ 37 & 53 & 54 & 40 & 39 \\ 38 & 54 & 55 & 41 & 40 \\ 39 & 55 & 56 & 42 & 41 \\ 40 & 57 & 58 & 44 & 43 \\ 41 & 58 & 59 & 45 & 44 \\ 42 & 59 & 60 & 46 & 45 \\ 43 & 60 & 61 & 47 & 46 \\ 44 & 61 & 62 & 48 & 47 \\ 45 & 62 & 63 & 49 & 48 \\ 46 & 63 & 64 & 50 & 49 \\ 47 & 64 & 65 & 51 & 50 \\ 48 & 65 & 66 & 52 & 51 \\ 49 & 66 & 67 & 53 & 52 \\ 50 & 67 & 68 & 54 & 53 \\ 51 & 68 & 69 & 55 & 54 \\ 52 & 69 & 70 & 56 & 55 \\ 53 & 71 & 72 & 58 & 57 \\ 54 & 72 & 73 & 59 & 58 \\ 55 & 73 & 74 & 60 & 59 \\ 56 & 74 & 75 & 61 & 60 \\ 57 & 75 & 76 & 62 & 61 \\ 58 & 76 & 77 & 63 & 62 \\ 59 & 77 & 78 & 64 & 63 \\ 60 & 78 & 79 & 65 & 64 \\ 61 & 79 & 80 & 66 & 65 \\ 62 & 80 & 81 & 67 & 66 \\ 63 & 81 & 82 & 68 & 67 \\ 64 & 82 & 83 & 69 & 68 \\ 65 & 83 & 84 & 70 & 69 \\ 66 & 85 & 86 & 72 & 71 \\ 67 & 86 & 87 & 73 & 72 \\ 68 & 87 & 88 & 74 & 73 \\ 69 & 88 & 89 & 75 & 74 \\ 70 & 89 & 90 & 76 & 75 \\ 71 & 90 & 91 & 77 & 76 \\ 72 & 91 & 92 & 78 & 77 \\ 73 & 92 & 93 & 79 & 78\end{array}$

Fig. 24. (continued) 


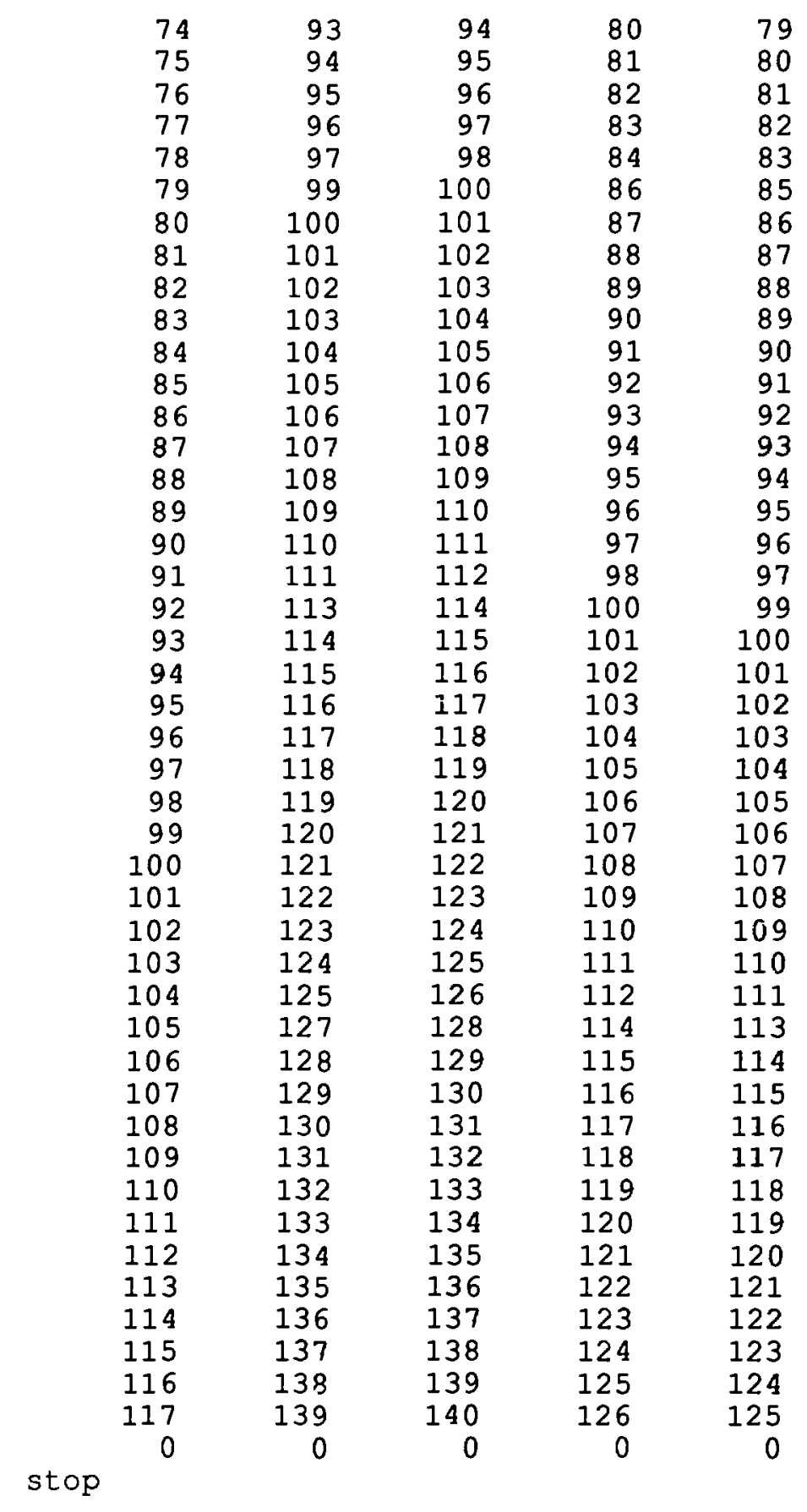

Fig. 24. (continued) 


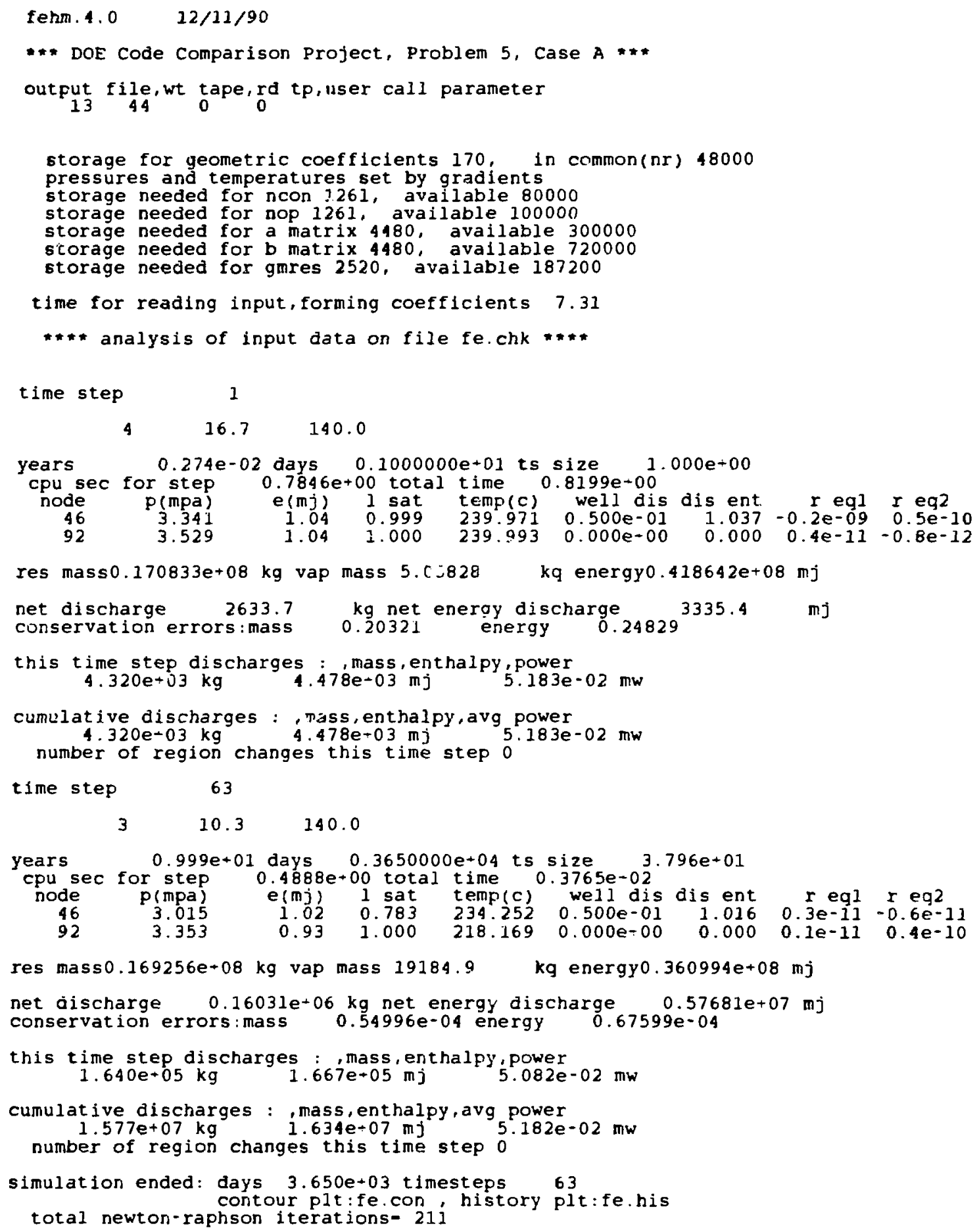

Fig. 25. Output from FEHMN for the DOE problem. 
temp contours for doe problem

00

03000200

00

6.55 .5

$1-1.0 .00 .00 .0$

8 160. 480. 200. 210. 220. 225. 230. 235.

Fig. 26. Instruction file (CON.INS) for the postprocessor FECPLTR for the DOE problem. 


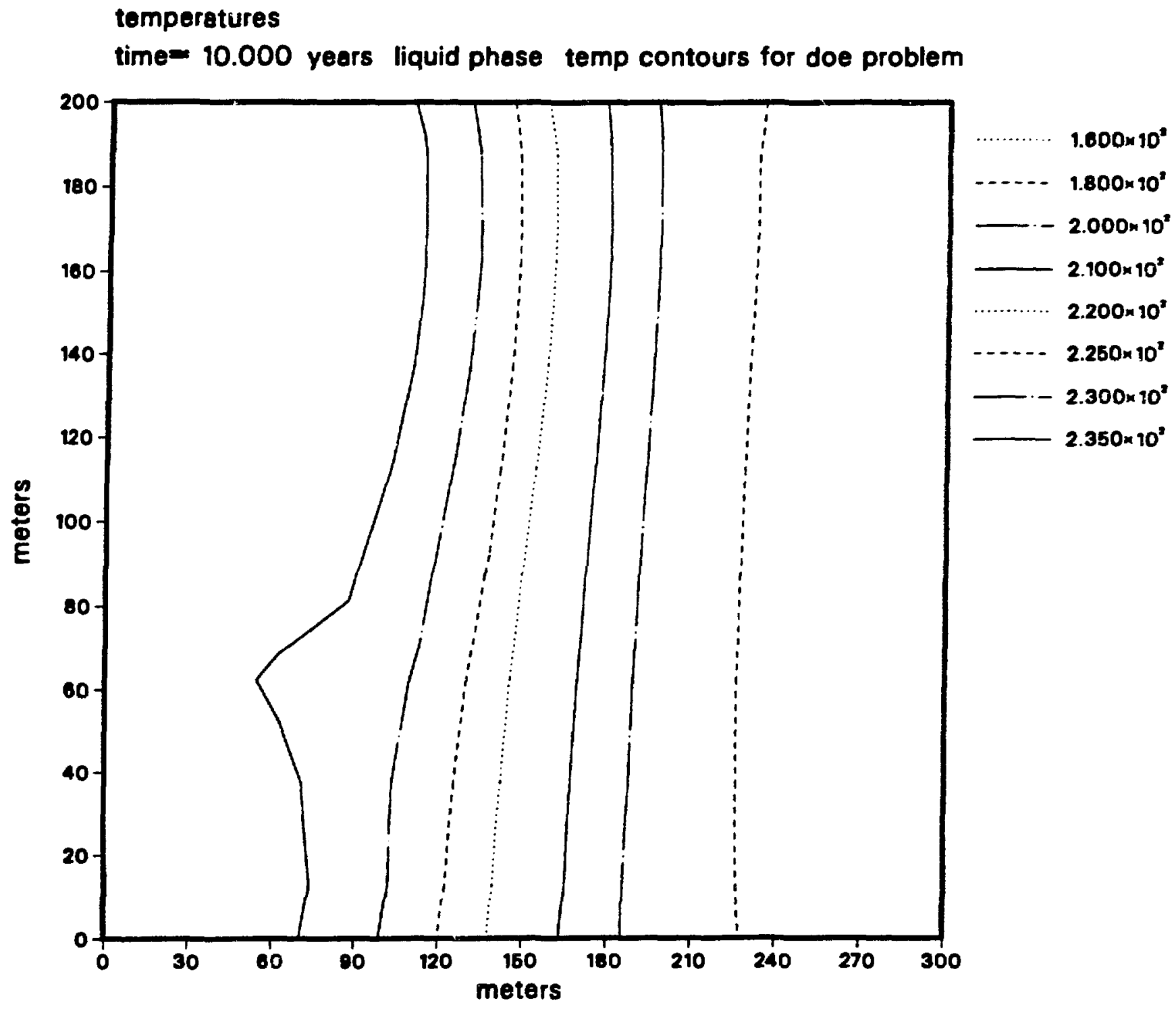

Fig. 27. Contour plot of temperature for the DOE problem. 


\section{ACKNOWLEDGMENTS}

The authors thank Kay Coen, Kay Birdsell, and Mary Ann Olson for their contributions to this document.

\section{DISCLAIMER}

The software documented in this report was not verified, validated, or otherwise subjected to the controls of a Yucca Mountain Site Characterization Project Office-approved quality program.

\section{REFERENCES}

Behie, A., and P. A. Forsythe, "Incomplete Factorization Methods for Fully Implicit Simulation for Enhanced Oil Recovery," SIAM, J. Sci. Stat. Comput. 5, 543-561 (1984). [NNA.910806.0004].

Behie, A., and P. K. W. Vinsome, "Block Iterative Methods for Fully Implicit Reservoir Simulations," Soc. Pet. Eng. J. 22, 658-668 (1982). [NNA.910806.0005].

Birdsell, K. H., K. Campbell, K. G. Eggert, and B. J. Travis, "Simulation of Radionuclide Retardation at Yucca Mountain Using a Stochastic Mineralogical/Geochemical Model," Proceedings of the First International Meeting on High Level Radioactive Waste Management, Las Vegas, Nevada, April 8-12, 1990. [NNA.900824.0091].

Brownell, D. H., S. K. Garg, and J. W. Pritchett, "Computer Simulation of Geothermal Reservoirs," Paper SPE 5381, Proceedings of the 45th California Regional Meeting of the Soc. Pet. Eng. of AIME, Ventura, California (1975). [NNA.910806.0006].

Corey, A. T., "The Interrelation Between Gas and Oil Relative Permeabilities," Prod. Mon. 19, 38-41 (1954). [NNA.900720.0036].

Dalen, V., "Simplified Finite-Element Models for Keservoir Flow Problems," Soc. Pet. Eng. J. 19, 333-343 (1979). [NNA.910806.0007].

Diaz, J. R., W. R. Jines, and T. Steihaug, "On the Convergence Criterion for Linear (Inner) Iterative Solvers for Reservoir Simulation," Paper SPE 13500 Proceedings of the Eighth SPE Symposium on Reservoir Simulation, Dallas, Texas, February (1985). [NNA.890523.0135].

Gangi, A. F., "Var:ation of Whole and Fractured Porous Rock Permeability with Confining Pressure," Rock Mech. Sci. and Geomech. Abstr. 15, 249-257 (1978). [NNA.900403.0014]. 
Hinton, E., and D. R. J. Owen, An lritroduction to Finite Element Computations (Pineridge Press, Swansea, Wales, 1979). [NNA.910806.0008].

Klavetter, E. A., and R. R. Peters, "Estimation of Hydrologic Properties of an Unsaturated Fractured Rock Mass," Sandia Report SAND84-2642, 1986. [HQS.880517.3118].

Meijerink, J. A., and H. A. Van der Vorst, "An Iterative Solution Method for Linear Systems of Which the Coefficient Matrix is a Symmetric M-Matrix," Mathematics of Computation 31, 148-162 (1977). [NNA.890523.0134].

Mercer, J. W. and C. R. Faust, "Simulation of Water- and Vapor-Dominated Hydrothermal Reservoirs," Paper SPE 5520, Proceedings of the 50th Annual Fall Meeting of the Soc. Pet. Eng. of AIME, Dallas, Texas (1975). [NNA.910806.0009].

Moench, A. F., "Double-Porosity Models for a Fissured Groundwater Reservoir with Fracture Skin," Water Resources Reference 20:7, 831-846 (1984). [HQS.880517.2762].

Molloy, M. W., "Geothermal Reservoir Engineering Code Comparison Project," Proceedings of the Sixth Workshop on Geothermal Reservoir Engineering, Stanford University (1980). [NNA.910806.0010].

Nitao, J., "Numerical Modeling of the Thermal and Hydrological Environment Around a Nuclear Waste Package Using the Equivalent Continuum Approximation: Horizontal Emplacement," Lawrence Livermore National Laboratory report UCID-21444(1988). [NNA.890317.0021].

Pruess, K., "TOUGH Users Guide, " Lawrence Berkeley Laboratory report LBL-20709 (June 1987). [NNA.890315.0010].

Robinson, B. "Sorption Column Modeling for $\mathrm{LiBr}$," Los Alamos document Group EES-4/7-88-1 (July 1988). [NNA.910806.0011].

Saad, Y., and M. H. Schultz, "GMRES: A Generalized Minimal Residual Algorithm for Solving Non-Symmetric Linear Systems," SIAM Journal of Scientific and Statistical Computation 7(3), 856-869 (1986). [NNA.890523.0137].

Satter, A., W. M. Shum, W. T. Adams, and L. A. Davis, "Chemical Transport in Porous Media with Dispersion and Rate-Controlled Adsorption," Soc. Pet. Eng. J. 20, 129-138, (1980). [NNA.910806.0012].

Thomas, L. K., and R. G. Pierson, "Three Dimensional Reservoir Simulation," Soc. Pet. Eng. J. 18, 151-161 (1978). [NNA.910806.0013]. 
Toronyi, R. M., and S. M. Faronq Ali, "Two-phase, Two-dimensional Simulation of a Geothermal Reservoir and Wellbore System," Soc. Pet. Eng. J. 17, 171-183 (1977). [NNA.910806.0014] .

van Genuchten, M. T. "A Closed Form Equation for Predicting Hydraulic Conductivity of Unsaturated Soils," Soil Sci. Soc. Am. J., 44, 892-898 (1980). [NNA.890522.0287] .

Warren, J. E., and P. J. Root, "The Behavior of Naturally Fractured Reservoirs," Soc. Pet. Eng. J. 3, 245-255 (1963). [NNA.890522.0276].

Weeks, E. P., "Effect of Topography on Gas Flow in Unsaturated Fractured Rock: Concepts and Observations," Proceedings of the American Geophysical Union Symposium on Flow and Transport in Unsaturated Fractured Rock, D. Evans and T. Nicholson, Eds., Geophysical Monograph 42, AGU (1987). [HQS.880517.3217].

Young, L. C., "A Finite Element Method for Reservoir Simulation," Soc. Pet. Eng. J. 21, 115-128 (1981). [NNA.910806.0015].

Zienkiewicz, O. C., The Finite Element Method (McGraw-Hill, London, 1977). [NNA.910327.0066].

Zienkiewicz, O. C., and C. J. Parekh, "Transient Field Problems: Two and Three Dimensional Analysis by Isoparametric Finite Elements," Int. J. Numer. Methods Eng. 2, 61-70 (1970). [NNA.910806.0016] .

Zyvoloski, G., "Finite Element Methods for Geothermal Reservoir Simulation," Int. J. Numer. Anal. Methods Geomech. 7, 75-86 (1983). [NNA.910806.0017].

Zyvoloski, G., "Incomplete Factorization for Finite Elements," International Journal for Numerical Methods in Engineering 23, 1101-1109, 1986. [NNA.890523.0138].

Zyvoloski, G. and Z. Dash, "Software Verification Report FEHMN Version 1.0," Los Alamos National Laboratory report LA-UR-91-609 (1991). [NNA.910806.0018].

Zyvoloski, G., Z. Dash, and S. Kelkar, "FEHM: Finite Element Heat and Mass Transfer Code," Los Alamos National Laboratory report LA-11224-MS (March 1988). [NNA.900918.0013]. 
The following number is for Office of Civilian Radioactive Waste Management (OCRWM) records management purposes only and should not be used when ordering this publication.

Accession Number NNA.910625.0038

This report has been reproduced directly from the best available copy.

It is available to DOE and DOE contractors from the Office of Scientific and Technical Information,

P.O. Box 62,

Oak Ridge, TN 37831

Prices are available from

(615) 576-8401, FTS 626-8401.

It is available to the public from the

National Technical Information Service,

U.S. Deparment of Commerce,

5285 Port Royal Rd.,

Springfield YA 22161 